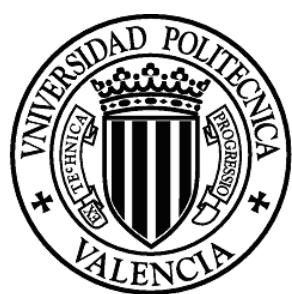

Universidad Politécnica de Valencia

Departamento de Matemática Aplicada

\title{
Métricas fuzzy. Aplicaciones al filtrado de imágenes en color
}

\author{
Memoria presentada por \\ Andrés LóPez CRevilléN \\ para optar al Grado de Doctor \\ por la Universidad Politécnica de Valencia \\ Dirigida por los Doctores \\ D. Valentín Gregori Gregori \\ D. Samuel Morillas Gómez
}

Gandía, febrero de 2010 

D. Valentín Gregori Gregori y D. Samuel Morillas Gómez, Profesores Doctores del Departamento de Matemática Aplicada de la Universidad Politécnica de Valencia

Certifican: que la presente memoria "Métricas fuzzy. Aplicaciones al filtrado de imágenes en color" ha sido realizada bajo su dirección por D. Andrés López Crevillén, en el Departamento de Matemática Aplicada de la Universidad Politécnica de Valencia, y constituye su tesis para optar al grado de Doctor.

Y para que así conste, presentan la referida tesis, firmando el presente certificado.

Valencia, 25 de febrero de 2010

Fdo. Valentín Gregori Gregori Fdo. Samuel Morillas Gómez 
A mis padres, Ana Mari y Andrés $\dagger$ 


\section{Agradecimientos}

Quiero expresar mi agradecimiento a todas aquellas personas que, de una forma $u$ otra, han ayudado a que esta tesis sea una realidad.

En primer lugar, quiero agradecer enormemente a los dos directores de esta tesis, Dr. Valentín Gregori Gregori y Dr. Samuel Morillas Gómez, todo el trabajo que han realizado y lo que me han enseñado, facilitándome siempre las cosas, depositando su confianza en mí y recibiendo de ellos en todo momento un trato excepcional. También quiero expresar mi agradecimiento a Almanzor, por toda la ayuda que me ha prestado siempre que la he necesitado.

Quiero agradecer profundamente a mis padres la educación que recibí de ellos, que sirvió para guiarme por un camino adecuado, estando siempre a mi lado en los momentos que más los he necesitado. También quiero agraceder a mis hermanos y a Miguel todo el apoyo que he recibido de ellos. No me puedo olvidar de mis abuelos $y$ de mis tíos, que me han servido de ejemplo en muchas ocasiones.

En esta página no podían faltar Fani y Joan, que junto con Paco y Encarnita fueron los culpables (en el buen sentido de la palabra) de que naciera este trabajo.

También quiero agradecer a mis compañeros de facultad, especialmente a David Crevillén y David Salar, los momentos que compartimos mientras estudiábamos.

Finalmente quiero hacer un agradecimiento muy especial a Gemma, por su incondicional apoyo, motivación, comprensión, cariño...

Sin todos vosotros esto no hubiera sido posible. Muchísimas gracias. 


\title{
Métricas fuzzy. Aplicaciones al filtrado de imágenes en color
}

\author{
Andrés López Crevillén
}

Uno de los problemas más importantes en Topología Fuzzy es obtener un concepto apropiado de espacio métrico fuzzy. Este problema ha sido abordado por muchos autores desde diferentes puntos de vista. En particular, es de gran interés la noción de métrica fuzzy sobre un conjunto que, con la ayuda de t-normas continuas, introdujeron y estudiaron George y Veeramani. En el presente trabajo hemos continuado con el estudio de estos espacios métricos fuzzy, hemos aportado nuevos ejemplos que nos ayudarán a desarrollar la teoría y hemos tratado otras cuestiones relacionadas con la convergencia, la continuidad y dos tipos de métricas fuzzy llamadas principales y fuertes.

En la presente tesis, aportamos nuevos ejemplos de métricas fuzzy y en algunos casos obtenemos que las métricas fuzzy más habituales en la literatura sobre el tema, son casos particulares de las que aquí damos. Tratamos también la extensión de dos métricas fuzzy estacionarias cuando coinciden en la intersección de dos conjuntos. También hemos continuado con el estudio del concepto de p-convergencia en espacios métricos fuzzy introducido por D. Mihet, estudiando algunos aspectos relativos a él y damos una caracterización de aquellos espacios métricos fuzzy, que llamamos principales, en los que la familia de las sucesiones p-convergentes coincide con la familia de las sucesiones convergentes. Además damos un ejemplo de espacio métrico fuzzy no completable y no principal. 
Definimos el concepto de aplicación t-continua entre espacios métricos fuzzy, que es más fuerte que el de continuidad y revisamos algunas cuestiones referentes a este tipo de aplicaciones, obteniendo como resultado que si el espacio métrico fuzzy de partida es principal entonces las aplicaciones tcontinuas coinciden con las aplicaciones continuas.

En lo que respecta al capítulo de métricas fuertes, estudiamos una clase de métricas fuzzy estacionarias que incluye la clase de las ultramétricas fuzzy estacionarias, que admite completación.

Por último, dado que en trabajos recientes se ha demostrado que las métricas fuzzy son interesantes para problemas de ingeniería y útiles en variedad de aplicaciones, estudiamos una aplicación de estas métricas en el filtrado de imágenes digitales en color. El proceso de filtrado de una imagen consiste en el reemplazamiento de los píxeles de la imagen ruidosa original por otros píxeles libres de ruido que se determinan mediante un proceso que involucra el uso de una métrica. En este contexto, usualmente se han utilizado métricas clásicas y nosotros estudiamos la utilización de ciertas métricas fuzzy. En una primera aplicación utilizamos cuatro métricas fuzzy y comparamos los resultados con las métricas clásicas $L_{2}$ y $L_{\infty}$. En la segunda aplicación, la métrica fuzzy que utilizamos se define como un producto de dos métricas fuzzy para así combinar dos criterios de distancia: cercanía espacial y similitud de las componentes de color RGB de un píxel. Ambas aplicaciones revelan que las métricas fuzzy son una herramienta prometedora para el procesamiento de imagen y, en general, para problemas de ingeniería. 


\title{
Mètriques fuzzy. Aplicacions al filtratge d'imatges en color
}

\author{
Andrés López Crevillén
}

Un dels problemes més importants en Topologia Fuzzy és obtindre un concepte adequat d'espai mètric fuzzy. Aquest problema ha sigut abordat per molts autors des de diferents tipus de vista. En particular, és de gran interés la noció de mètrica fuzzy sobre un conjunt que, amb l'ajuda de $t$ normes contínues, van introduir George i Veeramani. Al present treball hem continuat amb l'estudi dels espais mètrics fuzzy, hem aportat nous exemples que ens ajudaran a desenvolupar la teoria i hem tractat altres qüestions relacionades amb la convergència, la continuïtat i dos tipus de mètriques fuzzy anomenades principals i fortes.

A la present tesi, aportem nous exemples de mètriques fuzzy i en alguns casos obtenim que les mètriques fuzzy més habituals en la literatura sobre el tema, són casos particulars de les que ací donem. Tractem també l'extensió de dues mètriques fuzzy estacionàries quan coincideixen en la intersecció de dos conjunts. També hem continuat amb l'estudi del concepte de $p$-convergència en espais mètrics fuzzy introduït per D. Mihet, estudiant alguns aspectes relatius a ell i donem una caracterització d'aquells espais mètrics fuzzy, que anomenem principals, on la família de les successions $p$ convergents coincideix amb la família de les successions convergents. A més donem un exemple d'espai mètric fuzzy no completable i no principal. 
Definim el concepte d'aplicació $t$-contínua entre espais mètrics fuzzy, que és més fort que el de continuïtat i revisem algunes qüestions referents a aquest tipus d'aplicacions, obtenint com a resultat que si l'espai mètric fuzzy de partida és principal, llavors les aplicacions $t$-contínues coincideixen amb les aplicacions contínues.

Pel que fa al capítol de mètriques fortes, estudiem una classe de mètriques fuzzy estacionàries que inclou la classe de les ultram̀etriques fuzzy estacionàries, que admet completació.

Per últim, com que en treballs recents s'ha demostrat que les mètriques fuzzy són interessants per a problemes d'enginyeria i útils en una diversitat d'aplicacions, estudiem una aplicació d'aquestes mètriques al filtratge d'imatges digitals en color. El procés de filtratge d'una imatge consisteix en el reemplaçament dels píxels de la imatge sorollosa original per altres píxels lliures de soroll que es determinen mitjançant un procés que involucra l'ús d'una mètrica. En aquest context, usualment s'han utilitzat mètriques clàssiques i nosaltres estudiem l'ús de certes mètriques fuzzy. En una primera aplicació utilitzem quatre mètriques fuzzy i comparem els resultats amb les mètriques clàssiques $L_{2}$ i $L_{\infty}$. En la segona aplicació, la mètrica fuzzy que utilitzem es defineix com un producte de dues mètriques fuzzy per a així combinar dos criteris de distància: proximitat espacial i similitud de les components de color RGB d'un píxel. Ambdues aplicacions revelen que les mètriques fuzzy són una eina prometedora per al processat d'imatges i, en general, per a problemes d'enginyeria. 


\title{
Fuzzy metrics. Applications to colour image filtering
}

\author{
Andrés López Crevillén
}

One of the most important problems in Fuzzy Topology is to obtain an appropriate concept of fuzzy metric space. This problem has been approached by many authors and from different points of view. In particular, the notion of fuzzy metric on a set that, with the help of continuous t-norms was introduced by George and Veeramani is of great interest. In this dissertation, we have advanced in the study of fuzzy metric spaces, we have given new examples that will be helpful to develop theoretical issues, and we have approached other points related to convergence, to continuity and to two particular types of fuzzy metrics called principal and strong .

In this Thesis, we provide new examples of fuzzy metrics and, in some cases, we conclude that the most common fuzzy metrics in the literature are particular cases of those provided here. We also deal with the extension of two stationary fuzzy metrics when they coincide with the intersection of two sets. In addition, we have advanced in the study of the p-convergency concept in fuzzy metric spaces introduced by D. Mihet, where we have dealt with some related aspects and we have given a characterization for those fuzzy metric spaces that we call principal, in which the family of the p-convergent sequences coincide with the family of convergent sequences. Moreover, we provide an example of a non completable and non principal fuzzy metric space. 
We define the concept of $t$-continuous application between fuzzy metric spaces, which is stronger than the continuity concept, and we revise some aspects related to this kind of applications obtaining as a result, that, if the initial fuzzy metric space is principal, then the t-continuous applications coincide with the continuous applications.

With respect to the chapter of strong fuzzy metrics, we study a class of stationary fuzzy metrics that includes the class of stationary fuzzy ultrametrics, which admits completion.

Finally, provided that recent works have shown that fuzzy metrics are interesting for engineering problems and useful in a variety of applications, we study how fuzzy metrics can be used for colour image filtering. The image filtering process consists of the replacement of the pixels in a noisy original image with other noise-free pixels which are determined by means of a procedure that involves the usage of a metric. In this context, usually classical metrics have been employed and we study the usage of certain fuzzy metrics, instead. In a first application, we use four different fuzzy metrics for the filtering process and we compare the results with the classical $L_{2}$ and $L_{\infty}$ metrics. In a second application, we employ a novel fuzzy metric which is defined as the product of two fuzzy metrics in order to combine two different distance criteria: pixel spatial closeness and pixel RGB colour similarity. Both applications show that fuzzy metrics are a promising tool for image processing and, in general, for engineering problems. 


\section{Índice general}

Introducción general

1. Preliminares

1.1. Introducción . . . . . . . . . . . . . . . 15

1.2. Espacios métricos fuzzy de George y Veeramani . . . . . . . 17

2. Ejemplos de métricas fuzzy

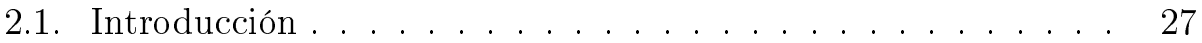

2.2. Generalización de métricas fuzzy conocidas . . . . . . . . 31

2.3. Métricas fuzzy definidas mediante métricas . . . . . . . 36

2.4. La métrica fuzzy discreta $\ldots \ldots \ldots$. . . . . . . . . . 42

2.5. Métricas fuzzy definidas por funciones simétricas . . . . . 43 
2.6. Métricas fuzzy definidas a partir de un par de funciones . . . 49

2.7. Métricas fuzzy definidas por t-normas continuas . . . . . . 53

2.8. Métricas fuzzy definidas por métricas fuzzy $\ldots \ldots \ldots 6$

2.9. Métricas fuzzy definidas por particiones $\ldots \ldots \ldots 6$

2.10. Extensión de una métrica fuzzy . . . . . . . . . . . 62

2.10.1. Extensión de dos métricas fuzzy coincidentes en su codominio . . . . . . . . . . . . . . 62

2.10.2. Métricas fuzzy definidas por isometrías . . . . . . . . 67

3. Métricas fuzzy principales

3.1. Introducción . . . . . . . . . . . . . . . . 69

3.2. Convergencia en espacios métricos fuzzy . . . . . . . . 71

3.3. Continuidad en espacios métricos fuzzy . . . . . . . . . . 83

4. Métricas fuzzy fuertes

4.1. Introducción . . . . . . . . . . . . . . . . . . . . 89

4.2. Una clase de espacios métricos fuzzy completables . . . . . . . 91

4.2.1. Métricas fuzzy fuertes. Completitud . . . . . . . . 91 
4.2.2. Completación de métricas fuzzy fuertes . . . . . . . . 104

5. Aplicación de métricas fuzzy

5.1. Introducción . . . . . . . . . . . . . . . . 113

5.2. Imágenes digitales . . . . . . . . . . . . . . . 115

5.3. Filtrado de imagen utilizando métricas fuzzy . . . . . . . . 117

5.4. Primera aplicación . . . . . . . . . . . . . . . . . 119

5.5. Segunda aplicación . . . . . . . . . . . . . . . . . . 122

Bibliografía 129 


\section{Introducción general}

El presente trabajo está dedicado al estudio de los espacios métricos fuzzy, en el sentido de George y Veeramani. Las líneas siguientes sitúan el trabajo dentro del contexto de la teoría fuzzy.

En 1965, Lofti A. Zadeh [56] introdujo el concepto de conjunto fuzzy, que transformó la mayoría de las ramas de la Ciencia y la Ingeniería (incluida la Matemática). Un conjunto fuzzy se define matemáticamente mediante la asignación a cada elemento de un conjunto un valor posible en el intervalo $[0,1]$ que representa su grado de pertenencia al conjunto fuzzy. Formalmente, dado un conjunto no vacío $X$, se denomina conjunto fuzzy de $X$ a toda aplicación $A: X \rightarrow[0,1]$. Esta noción elimina, de alguna forma, la frontera entre los elementos pertenecientes y los no pertenecientes, introduciendo "borrosidad" en dicha frontera. La aparición de este concepto está motivada por la gran cantidad de situaciones reales en las que los objetos no tienen un criterio totalmente definido de pertenencia. La unión e intersección de una familia de conjuntos fuzzy de $X$, se define como el supremo e ínfimo, respectivamente, de la familia de funciones.

Uno de los primeros campos fuzzy que aparece en la Matemática fue el de la Topología Fuzzy, iniciada por C. L. Chang en 1968 [8]. Para Chang, 
una topología $\tau$ en $X$ es una familia de conjuntos fuzzy de $X$ cerrada para uniones cualesquiera, y para intersecciones finitas, que contiene las funciones constantes 0 y 1 . Aunque éste es el concepto mayormente utilizado por los autores que posteriormente han desarrollado la teoría, no es el único. Así, en 1976, R. Lowen [37], [36], exige además que $\tau$ contenga todas las funciones constantes; a esta topología en [53] se la denomina laminada. En consecuencia, la topología laminada no constituye una generalización de la topología, en sentido clásico, a diferencia de la de Chang. Por otra parte, Goguen [18] y Hutton [31], generalizan la noción de Chang reemplazando el rango $I=[0,1]$ de los conjuntos fuzzy, por un retículo complementado $L$, dando lugar al concepto de $L$-topología.

En los anteriores casos los miembros de $\tau$, que se denominan abiertos, carecen de graduación. Esta situación cambia en la estructura topológica fuzzy que define A. Šostak [52], para el cual una topología fuzzy es una aplicación $\sigma: I^{X} \rightarrow I$, que verifica ciertos axiomas. A la misma noción, tras ligeras modificaciones, [9], [29], llegaron Hazra et al., denominando graduación de abiertos a la aplicación $\sigma$.

K. Atanassov [4], [3], [5] ha introducido el concepto de conjunto intuicionista, que generaliza el de conjunto fuzzy y que ha sido usado por D. Çoker [10] para definir el concepto de espacio topológico intuicionista, como una generalización del concepto de topología fuzzy debido a Chang.

Recientemente, J. H. Park [45] ha introducido y estudiado una noción de espacio métrico fuzzy intuicionista usando la idea de conjunto fuzzy intuicionista debido a Atanassov. En [25] los autores demostraron que para cada espacio métrico fuzzy intuicionista $(X, M, N, *, \diamond)$, la topología generada por la métrica fuzzy intuicionista $(M, N)$ coincide con la topología generada por la métrica fuzzy $M$, y por lo tanto, el estudio del espacio $(X, M, N, *, \diamond)$ se 
reduce al estudio del espacio métrico fuzzy $(X, M, *)$.

Uno de los problemas más importantes de la Topología Fuzzy es obtener un concepto apropiado de espacio métrico fuzzy. Recordemos que el estudio de los espacios métricos se basa en la noción de distancia entre puntos, pero en muchas ocasiones reales, esta distancia no puede determinarse con exactitud. Este problema, que pertence al ámbito fuzzy, había sido anteriormente abordado por la teoría de la probabilidad. En efecto, en 1942, K. Menger introdujo los llamados espacios métricos probabilísticos, donde si $d(x, y)$ es la distancia entre los puntos $x$ e $y$, entonces la función de distribución $F_{x y}(t)$ representa la probabilidad de que la distancia entre $x$ e $y$ sea menor que $t$. Posteriormente, Schweizer y Sklar, continuaron el desarrollo de estos espacios $\mathrm{y}$, recientemente han sido muchos los trabajos aportados en este sentido [6], [43], [44], [47], [51], [54], etc.

Como ya hemos visto, la noción de Topología Fuzzy ha sido tratada desde diversos puntos de vista, lo cual es casi una constante en cuantos conceptos fuzzy se definen. En lo que respecta a las métricas fuzzy, también han sido numerosos autores los que han abordado este concepto de distintas maneras, que nosotros, simplificando, dividimos en dos grandes grupos. El primero está formado por aquellos trabajos en los cuales una (pseudo-) métrica en $X$ es tratada como una función $d: \Omega \times \Omega \rightarrow \mathbb{R}$ con $\Omega \subset I^{X}(I=[0,1])$ satisfaciendo algunos axiomas análogos al caso clásico. Entre éstos podemos destacar los trabajos de Deng Zi-ke [57], Erceg [13], Hu [30], y Artico y Moresco [1]. Los principales problemas de interés en esta línea son: de qué manera una métrica fuzzy induce una (quasi-) uniformidad fuzzy (en el sentido de [32]) y una topología fuzzy [13], [57], [30]; criterios de (pseudo-) metrización [12], [13], [30]; propiedades de separación en espacios métricos [13], [1], [30], [58] y propiedades de completaciónn y acotación [1], [57]. En el segundo grupo incluimos aquellos artículos en los cuales la distancia entre objetos es fuzzy. 
Los más relevantes en esta dirección son los debidos a Kaleva y Seikkala [33], Eklund y Gähler [11], y Kramosil y Michalek [35]. El concepto de métrica fuzzy objeto de nuestro estudio es el debido a George y Veeramani [14], [16], que constituye una modificación del concepto de métrica fuzzy introducida por Kramosil y Michalek.

Kramosil y Michalek [35] definieron originalmente el concepto de espacio métrico fuzzy, que aparece ligeramente modificado en [20] y [14], de la siguiente forma. Se llama espacio métrico fuzzy de Kramosil y Michalek (KM-espacio métrico fuzzy) a una terna $(X, M, *)$ donde $X$ es un conjunto arbitrario, * es una t-norma continua y $M$ es un conjunto fuzzy en $X \times X \times[0,+\infty[$ que satisface las siguientes propiedades, para cualesquiera $x, y, z \in X$ y $t, s>0$ :

$(K M 1) M(x, y, 0)=0$

$(K M 2) M(x, y, t)=1$ para todo $t>0$ si y sólo si $x=y$

$(K M 3) M(x, y, t)=M(y, x, t)$

$(K M 4) M(x, y, t) * M(y, z, s) \leq M(x, z, t+s)$

$(K M 5) M(x, y, \cdot):[0,+\infty[\rightarrow[0,1]$ es continua por la izquierda

George y Veeramani [14], [16] modificaron los anteriores axiomas exigiendo que $M$ fuera positiva y continua respecto de $t$, y reemplazaron (KM2) por

$$
M(x, y, t)=1 \text { si y sólo si } x=y \text {. }
$$

De esta manera es obvio que toda métrica fuzzy sobre $X$ en el sentido de George y Veeramani lo es en el sentido de Kramosil y Michalek con tal de extenderla definiendo $M(x, y, 0)=0$ para todo $x, y \in X$. A partir de $M$ 
los autores dedujeron una topología $\tau_{M}$ sobre $X$ que era Hausdorff y además cumple el Primer Axioma de Numerabilidad. A partir de una métrica $d$ sobre un conjunto $X$ los autores definieron (Proposicion 1.2.5) la métrica fuzzy $M_{d}$ sobre $X$ dada por

$$
M_{d}(x, y, t)=\frac{t}{t+d(x, y)}
$$

a la cual denominaron estándar, y verifica que la topología deducida de $M_{d}$ coincide con la definida por $d$, con lo que todo espacio metrizable resulta ser fuzzy metrizable en el sentido obvio. Por otra parte, dichos autores modificaron la noción de sucesión de Cauchy introducida por Grabiec en el contexto de los espacios métricos fuzzy de Kramosil y Michalek, puesto que probaron que el espacio métrico fuzzy estándar deducido del espacio métrico usual no resultaba ser completo en el sentido de Grabiec [20]. Con esta nueva definición los autores probaron que dicho espacio es completo. Finalmente, los profesores Gregori y Romaguera [21] y los propios autores George y Veeramani [15], [17] han proseguido el estudio de dichos espacios. En [22], Gregori y Romaguera demostraron que la clase de espacios topológicos que son fuzzy metrizables coinciden con la clase de espacios topológicos metrizables. Este resultado permite reenunciar algunos teoremas clásicos sobre métricas completables y métricas (pre) compactas en el ámbito de los espacios métricos fuzzy [21]. Sin embargo, la teoría de la completación de métricas fuzzy es, en este contexto, muy diferente a la teoría clásica de completación de métricas: en efecto, existen espacios métricos fuzzy que no son completables (Ejemplo 2 de [22] y Ejemplo 2 de [23]). En el Teorema 1 de [23] Gregori y Romaguera dieron una caracterización de espacios métricos fuzzy completables.

Trabajos recientes han demostrado que las métricas fuzzy son interesantes para problemas de ingeniería y útiles en variedad de aplicaciones, en particular, en el filtrado de imágenes. Esto se debe principalmente a dos ventajas proporcionadas por las métricas fuzzy frente a las métricas clásicas: en 
primer lugar, los valores dados por las métricas fuzzy están en el intervalo ]0,1], independientemente de la naturaleza del concepto de distancia que se mida. Esto hace que diferentes criterios de distancia que inicialmente puedan estar en rangos muy distintos, sean fáciles de combinar, ya que las métricas fuzzy los llevan a un rango común. De esta manera, la combinación de varios criterios de distancia se puede hacer prácticamente de manera directa. En segundo lugar, las métricas fuzzy casan perfectamente con el empleo de otras técnicas fuzzy, ya que el valor dado por una métrica fuzzy puede ser empleado directamente, o interpretado, como un grado de cercanía. Esto hace posible incluir las métricas fuzzy, como parte de otros sistemas fuzzy complejos. Recientemente, las métricas fuzzy, se han aplicado al filtrado de imágenes en color proporcionando mejoras en algunos filtros al reemplazar las métricas clásicas [39], [40], [7], [41] y [42]. Lamentablemente, sólo unos pocos ejemplos de métricas fuzzy se encuentran en los artículos relativos a este tema, y por esta razón, en ocasiones, ciertas propiedades de métricas fuzzy que no han sido todavía establecidas, tampoco han podido ser refutadas.

En este trabajo hemos continuado con el estudio de algunos aspectos de los espacios métricos fuzzy en el sentido de George y Veeramani, aportando nuevos ejemplos y propiedades de estos espacios. Aunque cada uno de los capítulos que forman el trabajo van precedidos de una pequeña introducción, vamos a analizar a continuación los conceptos y resultados más relevantes que hemos obtenido en cada uno de ellos.

El capítulo 1 contiene los conceptos básicos que se necesitan para la comprensión del resto de la obra, a modo preliminar. Empezamos introduciendo la noción de métrica fuzzy $M$ sobre un conjunto $X$ (Definición 1.2.2) de George y Veeramani, que es la única que se utilizará en toda la obra bajo el término de métrica fuzzy, y se definen términos relativos a dicho concepto. Después se describe la topología $\tau_{M}$ sobre $X$ que se deduce de $M$. En la 
Proposición 1.2.13 se hace notar el buen comportamiento de la métrica fuzzy estándar $M_{d}$, en relación con los conceptos análogos del caso métrico. Introducido el término de sucesión de Cauchy (Definición 1.2.9), y los referentes a él, completitud y completación, se describen algunos resultados interesantes, entre ellos (Corolario 1.2.22) el que afirma que un espacio topológico es completamente metrizable si y sólo si es completamente fuzzy metrizable, en su sentido obvio. También se recuerda el concepto de isometría entre espacios métricos fuzzy, en el que los autores fundamentan después su teoría de completación de espacios métricos fuzzy [22] y [23]. Las métricas fuzzy que no dependen del parámetro real $t$ se denominan estacionarias (Definición 1.2.28) y tendrán un papel importante en capítulos posteriores. El capítulo concluye recordando las 3 t-normas continuas más utilizadas en el trabajo (la mínimo, el producto usual y la de Lukasievicz, denotada por $\mathfrak{L})$, y alguna relación entre ellas.

Tener variados ejemplos de métricas fuzzy es interesante para desarrollar la teoría de los espacios métricos fuzzy, y también para que puedan ser aplicados en el ámbito de la ingeniería. En el capítulo 2 aportamos nuevos ejemplos de métricas fuzzy que clasificamos a lo largo de las Secciones 2.2 - 2.9, que han sido denominadas atendiendo a la manera en que han sido definidas. Las métricas fuzzy habituales en la literatura sobre el tema, resultan ser casos particulares de las expuestas en el capítulo. En la Sección 2.10 tratamos la extensión de una métrica fuzzy desde dos puntos de vista distintos. El primer método (Proposición 2.10.1) permite extender a $H \cup K$, donde $H$ y $K$ son conjuntos no disjuntos, y en los que están definidas dos métricas fuzzy $M_{H} \mathrm{y}$ $M_{K}$ coincidentes en $H \cap K$, una métrica fuzzy $M$ que coincide con $M_{H}$ en $H$ y con $M_{K}$ en $K$, es decir, $\left.M\right|_{H}=M_{H}$ y $\left.M\right|_{K}=M_{K}$. El segundo, basado en el concepto de isometría (fuzzy) permite transportar la métrica fuzzy de un espacio a otro que estaba desprovisto de métrica fuzzy (Proposición 2.10.4) 
Como es sabido, las métricas fuzzy incluyen en su definición un parámetro $t$, lo cual permite introducir nuevos conceptos (en métricas fuzzy) con respecto a los conceptos de métricas clásicas. En este sentido, en [38], Mihet modificó la definición de convergencia y obtuvo un concepto más general llamado $p$-convergencia. (Una sucesión $\left\{x_{n}\right\}_{n \in \mathbb{N}}$ en $(X, M, *)$ se llama punto convergente o p-convergente a $x_{0} \in X$ si $\lim _{n \rightarrow \infty} M\left(x_{n}, x_{0}, t_{0}\right)=1$ para algún $\left.t_{0}>0\right)$. En el capítulo 3 se caracterizan los espacios métricos fuzzy, que llamamos principales, donde ambos conceptos, convergencia y p-convergencia, coinciden. (Un espacio métrico fuzzy $(X, M, *)$ es principal si $\{B(x, r, t): r \in] 0,1[\}$ es una base local de $x \in X$, para cada $x \in X$ y $t>0$ ). La mayoría de las métricas fuzzy conocidas en la literatura existente sobre el tema son principales. En la Sección 3.2 del capítulo 3, siguiendo las sugerencias que el autor nos da en [38], se introduce el concepto de p-sucesión de Cauchy (Definición 3.2.13) y se muestra que las p-sucesiones de Cauchy y las sucesiones de Cauchy son dos conceptos diferentes, incluso en espacios métricos fuzzy principales (Ejemplo 3.2.18). Se dan además algunos ejemplos ilustrativos, incluyendo un espacio métrico fuzzy no principal, que no es completable (Ejemplo 3.2.12). (El contenido de esta sección ha sido publicado en [26]).

En [28], Gregori y Sapena introdujeron las aplicaciones t-uniformemente continuas que fueron estudiadas más tarde en [24]. En la Sección 3.3 del capítulo 3 se revisa el concepto de continuidad entre espacios métricos fuzzy y se define y estudia el concepto de aplicación t-continua (acorde con el concepto de t-uniformemente continua), que es un concepto próximo al de aplicación continua. (Una aplicación entre espacios métricos fuzzy $f:(X, M) \longrightarrow$ $(Y, N)$ es t-continua en $x_{0} \in X$ si dados $\left.\varepsilon \in\right] 0,1[$ y $t>0$ existe $\delta \in] 0,1[$ tal que si $M\left(x_{0}, x, t\right)>1-\delta$, entonces $\left.N\left(f\left(x_{0}\right), f(x), t\right)>1-\varepsilon\right)$. Es obvio que, si una aplicación $f$ es t-continua en un punto $x_{0}$ entonces es continua en $x_{0}$, pero el recíproco es falso en general, como podemos ver en el Ejemplo 3.3.4. 
No obstante, demostraremos (Proposición 3.3.6) que si el espacio métrico fuzzy de partida de una aplicación $f$ continua en $x_{0}$ es principal, entonces $f$ es t-continua en $x_{0}$. En particular, si el espacio de partida es $M_{d}$ (la métrica fuzzy estándar) toda aplicación t-continua en un punto $x_{0}$ es continua en $x_{0}$, pues $M_{d}$ es principal (Corolario 3.3.7). (El contenido de esta sección ha sido publicado en [27]).

En la primera parte del capítulo 4 se analizan algunos aspectos de una clase de métricas fuzzy que llamamos fuertes, las cuales se obtiene mediante la inclusión de una nueva condición para este tipo de métricas fuzzy. Se dice que la métrica fuzzy $(M, *)$ es una métrica fuzzy fuerte si para cada $x, y, z \in X$ y $t>0$ cumple que

$$
M(x, z, t) \geq M(x, y, t) * M(y, z, t) .
$$

A toda métrica fuzzy $M$ se le asocia una familia $\left\{M_{t}: t>0\right\}$ de métricas fuzzy estacionarias y caracterizamos cuándo una familia arbitraria $\left\{M_{t}: t>0\right\}$ de métricas fuzzy estacionarias define una métrica fuzzy fuerte a través de la expresión $M(x, y, t)=M_{t}(x, y), \forall x, y \in X, t>0$. Se obtiene que una métrica fuzzy fuerte $M$ es completa si y sólo si $M_{t}$ es completa para todo $t>0$ (Corolario 4.2.10). Después, en la segunda parte del capítulo 4 se estudia el comportamiento de cierto tipo de métricas fuzzy que denominamos íntegras. (Decimos que una t-norma continua $*$ es íntegra si cumple que $a * b \neq 0$ siempre que $a, b \neq 0)$. Después demostramos que las métricas fuzzy estacionarias íntegras son completables (Corolario 4.2.15), y que si $(X, M, *)$ es un espacio métrico fuzzy fuerte completable, entonces también lo es $\left(X, M_{t}, *\right)$ para todo $t>0$ (Teorema 4.2.18), siendo falso el recíproco (Ejemplo 4.2.19).

En el capítulo 5, estudiamos una aplicación de métricas fuzzy para fil- 
trado de imágenes en color. El problema consiste en sustituir los píxeles defectuosos (o ruidosos) de la imagen de entrada, por otros libres de ruido. En el proceso de selección de los píxeles libres de ruido, comúnmente se utilizan distancias clásicas. En una primera aplicación, proponemos reemplazar estas distancias clásicas por métricas fuzzy que son casos particulares de algunas de las estudiadas en el Capítulo 2 y estudiamos las mejoras obtenidas con este reemplazamiento. En la segunda aplicación empleamos una métrica fuzzy que combina dos criterios de distancia, que son, la similitud del color y la proximidad espacial. Estudiaremos cómo esta métrica combinada implica mejoras significativas en la calidad de las imágenes filtradas. 


\section{Capítulo 1}

\section{Preliminares}

\subsection{Introducción}

Este capítulo contiene los conceptos básicos que se necesitan para la comprensión del resto de la obra, así como algunos resultados interesantes que se han obtenido por otros autores, relativos a los conceptos tratados.

$\mathrm{Al}$ principio establecemos el concepto de espacio métrico fuzzy, objeto de nuestro estudio, que es el debido a George y Veeramani, en la Definición 1.2 .2 , y presentamos la métrica fuzzy estándar $\left(M_{d}, \cdot\right)$ que se obtiene a través de una métrica $d$ sobre $X$ y que viene definida por

$$
M_{d}(x, y, t)=\frac{t}{t+d(x, y)}
$$

George y Veeramani en [14] probaron que toda métrica fuzzy $M$ en $X$ genera una topología $\tau_{M}$ en $X$ que tiene como base la familia de bolas abiertas 
con centro $x$, radio $r$ y parámetro $t$,

$$
\left\{B_{M}(x, r, t): x \in X, 0<r<1, t>0\right\}
$$

donde

$$
B_{M}(x, r, t)=\{y \in X: M(x, y, t)>1-r\} .
$$

Además demostraron que $\left(X, \tau_{M}\right)$ es un espacio Hausdorff que verifica el Primer Axioma de Numerabilidad. En las Definiciones 1.2.9 y 1.2.10 podemos ver los conceptos de sucesión de Cauchy en un espacio métrico fuzzy y de espacio métrico fuzzy completo.

La Proposición 1.2.13 muestra el buen comportamiento de la métrica fuzzy estándar respecto los conceptos métricos fuzzy que se establecen y los correspondientes conceptos clásicos. (Este buen comportamiento de la métrica fuzzy estándar veremos en lo sucesivo que es una constante, en cuantos conceptos interviene). En particular la topología $\tau_{M_{d}}$ deducida de $M_{d}$ y la deducida de la métrica $d$ coinciden; también las sucesiones de Cauchy que lo son para $M_{d}$ lo son para $d$, y recíprocamente.

Los profesores Gregori y Romaguera demostraron que la clase de los espacios topológicos metrizables coincide con la clase de los espacios topológicos fuzzy metrizables (Definición 1.2.16). Los resultados se pueden ver establecidos en el Teorema 1.2.17 y en el Corolario 1.2.18.

En la Definición 1.2.24 mostramos el concepto de isometría entre espacios métricos fuzzy que utilizaremos más adelante para el tratamiento de la completación de espacios métricos fuzzy. En la Definición 1.2.28 podemos ver el concepto de espacio métrico fuzzy estacionario, caracterizado porque su métrica fuzzy no depende del parámetro $t$. Ambos conceptos los presentaron Gregori y Romaguera en [22] y [23] respectivamente. 
Para finalizar este capítulo de preliminares, vemos las 3 t-normas continuas que utilizaremos a lo largo de todo el trabajo y que son las más comunmente usadas en la lógica fuzzy, y damos a conocer una serie de propiedades que nos serán de utilidad más adelante. Dichas t-normas son la t-norma del mínimo, el producto usual y la t-norma de Lukasievicz, que denotaremos por $\wedge, \cdot$ y $\mathfrak{L}$, respectivamente.

\subsection{Espacios métricos fuzzy de George y Veerama- ni}

Definición 1.2.1. Una operación binaria $*:[0,1] \times[0,1] \rightarrow[0,1]$ se dice que es una t-norma continua si satisface las siguientes condiciones:

(i) * es continua, asociativa y conmutativa

(ii) $a * 1=$ a para todo $a \in[0,1]$

(iii) $a * b \leq c * d$ siempre que $a \leq c$ y $b \leq d \quad a, b, c, d \in[0,1]$

Definición 1.2.2. Un espacio métrico fuzzy, en el sentido de George y Veeramani [14], es una terna $(X, M, *)$ donde $X$ es un conjunto no vacio, * es una $t$-norma continua y $M$ es un conjunto fuzzy de $X \times X \times] 0,+\infty[$ (es decir, $M$ es una aplicación de dicho conjunto con valores en el intervalo [0,1]) que satisface las siguientes condiciones, para cualesquiera $x, y, z \in X, t, s>0$ :

(GV1) $M(x, y, t)>0$

(GV2) $M(x, y, t)=1$ si $y$ sólo si $x=y$ 
(GV3) $M(x, y, t)=M(y, x, t)$

(GV4) $M(x, y, t) * M(y, z, s) \leq M(x, z, t+s)$

$(G V 5) M(x, y,-):] 0,+\infty[\rightarrow] 0,1]$ es continua

A partir de ahora denominaremos $M_{x y}(t)$ a la función del axioma $(G V 5)$ y por tanto para cualquier $x, y \in X$ se tiene que $M_{x y}(t)=M(x, y, t)$ es una función continua respecto de $t$.

El axioma $(G V 2)$ es equivalente a las siguientes dos condiciones:

$(G V 2)^{\prime} M(x, y, t)=1$, para algún $t>0$ implica $x=y$.

$(G V 2)^{\prime \prime} M(x, x, t)=1$ para todo $t>0$.

Si $(X, M, *)$ es un espacio métrico fuzzy, diremos que $(M, *)$ es una métrica fuzzy sobre $X$. También diremos que $(X, M)$ es un espacio métrico fuzzy o $M$ es una métrica sobre $X$. En ocasiones, diremos que $X$ es un espacio métrico fuzzy, si no existe lugar a confusión. Notar que la condición (GV4) es una versión fuzzy de la desigualdad triangular. Los valores $M(x, y, t)$ se consideran como el grado de cercanía de $x$ a $y$ con respecto a $t$ y por el axioma $(G V 2)$ podemos relacionar los valores 0 y 1 de una métrica fuzzy con las nociones de $\infty$ y 0 de una métrica clásica, respectivamente.

En ocasiones, sin mención explícita, utilizaremos los siguientes resultados que fueron dados en [14] para un espacio métrico fuzzy $(X, M, *)$, y una tnorma continua $*$, respectivamente.

Proposición 1.2.3. La función $M(x, y,-)=M_{x y}(t)$ es no decreciente, para todo $x, y \in X$. 
Por la continuidad de $*$ se puede probar el siguiente resultado.

Proposición 1.2.4. Sea $*$ una t-norma continua, entonces:

(i) Dado $r \in] 0,1[$ existe $s \in] 0,1[$ tal que $s * s \geq r$

(ii) Dados $r, s \in] 0,1[$ con $r>s$ existe $t \in] 0,1[$ tal que $r * t \geq s$

Proposición 1.2.5. Sea $(X, d)$ un espacio métrico. Consideremos $a * b=a b$, para cualesquiera $a, b \in[0,1]$, sea $M_{d}$ la función definida en el conjunto $X \times X \times] 0,+\infty[$ de la siguiente forma:

$$
M_{d}(x, y, t)=\frac{t}{t+d(x, y)}
$$

Entonces $\left(X, M_{d}, *\right)$ es un espacio métrico fuzzy.

Definición 1.2.6. La anterior métrica fuzzy $M_{d}$, inducida por la métrica $d$, se denomina estándar y a $\left(X, M_{d}, *\right)$ se le llama espacio métrico fuzzy estándar (asociado al espacio métrico $(X, d)$ ).

Nota 1.2.7. En el caso en que d sea la métrica euclídea de $\mathbb{R}$, a $M_{d}$ se le denominará métrica fuzzy euclídea de $\mathbb{R}$.

Nota 1.2.8. George y Veeramani demostraron en [14] que toda métrica fuzzy $M$ en $X$ genera una topología $\tau_{M}$ en $X$ que tiene como base la familia de bolas abiertas con centro $x$, radio $r$ y parámetro $t$,

$$
\left\{B_{M}(x, r, t): x \in X, 0<r<1, t>0\right\}
$$

donde

$$
B_{M}(x, r, t)=\{y \in X: M(x, y, t)>1-r\}
$$

Cuando no haya posibilidad de confusión, escribiremos $B(x, r, t)$ en lugar de $B_{M}(x, r, t)$. 
Los autores demostraron [14] que $\left(X, \tau_{M}\right)$ es un espacio de Hausdorff que verifica el Primer Axioma de Numerabilidad.

Definición 1.2.9. [14] Una sucesión $\left\{x_{n}\right\}_{n \in \mathbb{N}}$ en un espacio métrico fuzzy $(X, M, *)$ se dice que es de Cauchy si para cada $\varepsilon>0$ y $t>0$, existe $n_{0} \in \mathbb{N}$ tal que $M\left(x_{n}, x_{m}, t\right)>1-\varepsilon$, para cualesquiera $n, m \geq n_{0}$.

En ciertos contextos resulta cómodo decir que $\left\{x_{n}\right\}_{n \in \mathbb{N}}$ es M-Cauchy, y en ocasiones así lo haremos nosotros, en vez de decir que es de Cauchy en $(X, M)$.

Definición 1.2.10. Un espacio métrico fuzzy $(X, M, *)$ en el que toda sucesión de Cauchy converge se dice que es un espacio métrico fuzzy completo, y en tal caso diremos que $M$ es una métrica fuzzy completa en $X$.

Definición 1.2.11. Un espacio métrico fuzzy $(X, M, *)$ es compacto si el espacio topológico $\left(X, \tau_{M}\right)$ es compacto.

Definición 1.2.12. [14] Sea $(M, *)$ una métrica fuzzy en un conjunto $X$. Diremos que $X$ es F-acotado si existen $r \in] 0,1[y t>0$ tal que $M(x, y, t)>$ $1-r$ para cualesquiera $x, y \in X$.

En tal caso también diremos que $M$ es una métrica fuzzy F-acotada en $X$.

En cierto sentido puede decirse que el espacio métrico fuzzy estándar hereda las propiedades del espacio métrico asociado de procedencia. Más explícitamente se tiene la siguiente proposición.

Proposición 1.2.13. Sea $(X, d)$ un espacio métrico. Entonces:

(i) La topología $\tau(d)$ generada por la métrica d es la misma que la topología $\tau_{M_{d}}$ generada por su respectiva métrica fuzzy estándar asociada $M_{d}$. 
(ii) La sucesión $\left\{x_{n}\right\}_{n \in \mathbb{N}}$ es una sucesión de d-Cauchy (i.e. es una sucesión de Cauchy en el espacio métrico $(X, d)$ ) si y sólo si es una sucesión de Cauchy en el espacio métrico fuzzy estándar $\left(X, M_{d}, *\right)$ asociado a d.

(iii) Un subconjunto $A \subset X$ es acotado en el espacio métrico $(X, d)$ si $y$ sólo si $A$ es $F$-acotado en el espacio métrico fuzzy estándar $\left(X, M_{d}, *\right)$.

Teorema 1.2.14. [14] Una sucesión $\left\{x_{n}\right\}_{n \in \mathbb{N}}$ en un espacio métrico fuzzy $(X, M, *)$ converge a $x \in X$ si y sólo si $\lim _{n \rightarrow \infty} M\left(x_{n}, x, t\right)=1, \forall t>o$.

Es obvio que toda sucesión convergente es de Cauchy.

Proposición 1.2.15. [16] El espacio métrico $(X, d)$ es completo si y sólo si el espacio métrico fuzzy estándar $\left(X, M_{d}, *\right)$ es completo.

Definición 1.2.16. Un espacio topológico $(X, \tau)$ es fuzzy metrizable si existe una métrica fuzzy $M$ en $X$ tal que $\tau=\tau_{M}$.

Con esta definición se tiene, por la Proposición 1.2.13, que todo espacio topológico metrizable es fuzzy metrizable. Además, en [21], los autores probaron que también se verifica el recíproco y, en consecuencia, que la clase de los espacios topológicos metrizables coincide con la clase de los espacios topológicos fuzzy metrizables. Los resultados son los siguientes.

Teorema 1.2.17. [21] Sea $(X, M, *)$ un espacio métrico fuzzy. Entonces $\left(X, \tau_{M}\right)$ es un espacio topológico metrizable.

En la prueba del resultado anterior, los autores demostraron que la familia $\left\{U_{n}: n \in N\right\}$, donde $U_{n}=\left\{(x, y) \in X \times X: M\left(x, y, \frac{1}{n}\right)>1-\frac{1}{n}\right\}$, es una base (numerable) de una uniformidad $U_{M}$ en $X$, compatible con la topología 
deducida de la métrica fuzzy, que, a partir de ahora denominaremos uniformidad deducida de $(M, *)$. Como consecuencia de este resultado se obtiene el siguiente corolario.

Corolario 1.2.18. [21] Un espacio topológico es metrizable si y sólo si es fuzzy metrizable.

Así pues queda establecido, que la clase de los espacios topológicos metrizables coincide con la clase de los espacios topológicos fuzzy metrizables. Como consecuencia de ello se tiene el siguiente resultado.

Corolario 1.2.19. [16] Todo espacio métrico fuzzy separable verifica el Segundo Axioma de Numerabilidad.

Recordemos que un espacio topológico se dice completamente metrizable si admite una métrica (compatible) completa. Usaremos una terminología análoga para un espacio fuzzy metrizable.

Definición 1.2.20. Sea $(X, \tau)$ un espacio topológico. Diremos que $(X, \tau)$ es completamente fuzzy metrizable si admite una métrica fuzzy (compatible) completa.

A continuación enunciaremos resultados obtenidos en relación con el concepto de metrización fuzzy, que son versiones fuzzy de sus análogos en la teoría clásica que pueden encontrarse en [12].

Teorema 1.2.21. [21] Sea $(X, M, *)$ un espacio métrico fuzzy completo. Entonces $\left(X, \tau_{M}\right)$ es completamente metrizable.

Corolario 1.2.22. [21] Un espacio topológico es completamente metrizable si y sólo si es completamente fuzzy metrizable. 
Además, es sabido que todo espacio completamente metrizable es de Baire. En consecuencia, por el teorema anterior, se deduce el siguiente corolario.

Corolario 1.2.23. [14] Todo espacio métrico fuzzy completo es de Baire.

Definición 1.2.24. [22] Sean $(X, M, *)$ e $(Y, N, \star)$ dos espacios métricos fuzzy. Entonces:

a) Una función $f$ de $X$ en $Y$ se llama isometría si para cada $x, y \in X y$ $t>0, M(x, y, t)=N(f(x), f(y), t)$.

b) $(X, M, *)$ e $(Y, N, \star)$ se llaman isométricos si existe una isometría biyectiva de $X$ en $Y$.

Definición 1.2.25. [22] Sea $(X, M, *)$ un espacio métrico fuzzy. Una completación métrica fuzzy de $(X, M, *)$ es un espacio métrico fuzzy completo $(Y, N, \star)$ tal que $(X, M, *)$ es isométrico a un subespacio denso de $(Y, N, \star)$.

Definición 1.2.26. Un espacio métrico fuzzy $(X, M, *)$ se llama completable si admite una completación métrica fuzzy.

Proposición 1.2.27. [22] Si un espacio métrico fuzzy $(X, M, *)$ tiene una completación métrica fuzzy entonces dicha completación es única salvo isometría.

Si $\left(X^{*}, M^{*}, \star\right)$ es una completación métrica fuzzy de $(X, M, *)$. Atendiendo a la proposición anterior y a la construcción de la completación [23], podemos considerar que $X \subset X^{*}, \star$ es $*$ y que $M^{*}$ está definida sobre $X^{*}$ por

$$
M^{*}(x, y, t)=\lim _{n \rightarrow \infty} M\left(x_{n}, y_{n}, t\right)
$$

para todo $x, y, \in X^{*}, t>0$, donde $\left\{x_{n}\right\}_{n \in \mathbb{N}}$ e $\left\{y_{n}\right\}_{n \in \mathbb{N}}$ son sucesiones en $X$ que convergen a $x$ e $y$, respectivamente. 
Definición 1.2.28. [23] Un espacio métrico fuzzy $(X, M, *)$ se dice que es estacionario si $M$ no depende de $t$, es decir, si para cada $x, y \in X$, la función $M_{x y}(t)=M(x, y, t)$ es constante. También se dice en ocasiones que $M$ es estacionaria.

Si $M$ satisface los axiomas $(G V 1)-(G V 4)$ y no depende de $t$, obviamente satisface $(G V 5)$ y por lo tanto se trata de una métrica fuzzy estacionaria.

Si $(X, M, *)$ es un espacio métrico fuzzy estacionario, escribiremos $M(x, y)$ en vez de $M(x, y, t)$ y $B_{M}(x, \varepsilon)$ en vez de $B_{M}(x, \varepsilon, t)$ si no da lugar a confusión.

Denotaremos $*$ a una t-norma continua cualquiera. La t-norma minimo la denotaremos por $\wedge$, el producto usual por · y la t-norma de Lukasievicz por $\mathfrak{L}$, es decir,

$$
\begin{gathered}
a \wedge b=\min \{a, b\} \\
a \cdot b=a b \\
a \mathfrak{L} b=\max \{a+b-1,0\}
\end{gathered}
$$

Estas 3 t-normas son las más comúnmente usadas en lógica fuzzy, satisfacen las propiedades que enunciamos en la siguiente proposición y nos serán de utilidad más adelante.

Proposición 1.2.29. Con las notaciones anteriores se satisfacen las siguientes desigualdades:

(i) $a \mathfrak{L} b \leq a \cdot b \leq a \wedge b$, para todo $a, b \in[0,1]$

(ii) $a * b \leq a \wedge b$ para cualquier $t$-norma $*$ (continua) y para todo $a, b \in$ $[0,1]$. 
En ocasiones también se utilizará el siguiente lema, aunque no se haga mención explícita.

Lema 1.2.30. [49] Sea $X$ un conjunto arbitrario no vacio. Si $(M, *)$ es una métrica fuzzy en el conjunto $X y \star$ es una t-norma continua de manera que $\star \leq *$, entonces $(M, \star)$ es una métrica fuzzy en $X$.

En consecuencia, si $(M, \wedge)$ es una métrica fuzzy sobre $X$ entonces $(M, *)$ es una métrica fuzzy sobre $X$ para cualquiera que sea la t-norma continua $*$.

En lo que sigue, $\mathbb{R}, \mathbb{R}^{+}$y $\mathbb{N}$, denotarán los conjuntos de los números reales, números reales positivos y números enteros positivos respectivamente.

Nuestras referencias básicas para la topología general son [34] y [12]. 


\section{Capítulo 2}

\section{Ejemplos de métricas fuzzy}

En todo este capítulo, $X$ denotará un conjunto no vacío y $M$ una función definida sobre $X \times X \times \mathbb{R}^{+}$con valores en $\left.] 0,1\right]$.

\subsection{Introducción}

La escasez de ejemplos conocidos de métricas fuzzy ha venido impidiendo en ocasiones encontrar diferencias significativas entre la teoría de los espacios métricos y la teoría de los espacios métricos fuzzy, en el sentido de George y Veeramani, dado que, muchos ejemplos conocidos de métricas fuzzy, venían deducidos, de alguna manera, de métricas usuales, y ello hacía que sus propiedades fueran, en gran parte, heredadas. En este capítulo vamos a presentar nuevos ejemplos de métricas fuzzy que hemos clasificado según el modo en que han sido obtenidas. La mayoría de las métricas fuzzy existentes son casos particulares de las que aquí presentamos. 
Las Secciones 2.2 - 2.9 han sido denominadas según la forma en que se han definido las métricas fuzzy. En cada uno de los ejemplos de métrica fuzzy $(M, *)$ que presentamos, se establece para cuál de las 3 t-normas continuas que trabajamos (mínimo, producto usual y Lukasievicz) resulta ser $M$ métrica fuzzy, y damos un ejemplo que prueba que para otra t-norma, digamos mayor, (si * no es la t-norma mínimo) deja de serlo.

En la Sección 2.2, presentamos la métrica fuzzy $M$ dada por

$$
M(x, y, t)=\left(\frac{(\min \{f(x), f(y)\})^{\alpha}+g(t)}{(\max \{f(x), f(y)\})^{\alpha}+g(t)}\right)^{\beta}
$$

donde $f: X \rightarrow \mathbb{R}^{+}$es una función inyectiva, $g: \mathbb{R}^{+} \rightarrow[0,+\infty[$ una función continua y creciente y $\alpha, \beta>0$, la cual es una generalización, por ejemplo, de las métricas fuzzy

$$
M(x, y, t)=\frac{\min \{x, y\}+t}{\max \{x, y\}+t},
$$

y

$$
M(x, y)=\frac{\min \{x, y\}}{\max \{x, y\}}
$$

definidas sobre $X=\mathbb{R}^{+}$, que utilizaremos en capítulos sucesivos.

En la Sección 2.3 vemos algunas métricas fuzzy definidas mediante métricas clásicas. Entre ellas,

$$
M(x, y, t)=\frac{g(t)}{g(t)+m \cdot d(x, y)}, m \in \mathbb{R}^{+}
$$

que es una generalización de la métrica fuzzy estándar $M_{d}$.

En la Sección 2.4, consideramos una función $\left.\varphi: \mathbb{R}^{+} \rightarrow\right] 0,1[$ que es continua y creciente. La función $M$ definida por

$$
M(x, y, t)= \begin{cases}1, & \text { si } x=y \\ \varphi(t), & \text { si } x \neq y\end{cases}
$$


es una métrica fuzzy con la t-norma mínimo $\wedge$ sobre $X$.

Como caso particular, si $\varphi(t)=k$, donde $k \in] 0,1[$, se obtiene

$$
M(x, y, t)= \begin{cases}1, & \text { si } x=y \\ k, & \text { si } x \neq y\end{cases}
$$

que es una métrica fuzzy que denominados discreta. La topología asociada a dicha métrica es la topología discreta.

En la Sección 2.5, las métricas las definimos mediante funciones simétricas. Un ejemplo de este tipo lo damos utilizando la función (simétrica) $F(x, y)=\cos ^{2} x-\operatorname{sen}^{2} y$, que como veremos es métrica fuzzy para la t-norma $\mathfrak{L}$ de Lukasievicz pero no lo es para la t-norma producto.

En la Sección 2.6, las métricas están definidas a partir de un par de funciones que cumplen alguna determinada condición. Una métrica de este tipo es la de la Proposición 2.6.1, que podemos ver a continuación.

Sean $g, h: X \rightarrow] 0,1[$ funciones tales que

$$
\sup \{g(x): x \in X\}<\inf \{h(x): x \in X\}
$$

de manera que $(g+h)(x)=c$. Definimos la función $M$ por

$$
M(x, y)= \begin{cases}1, & \text { si } x=y \\ h(x)-g(y), & \text { si } x \neq y\end{cases}
$$

Entonces $(M, \mathfrak{L})$ es una métrica fuzzy sobre $X$.

En la Sección 2.7 nos encontramos con métricas fuzzy definidas mediante t-normas continuas. Un caso de éstas se puede ver en la Proposición 2.7.1 y 
como caso particular obtenemos que

$$
M(x, y)= \begin{cases}1, & \text { si } x=y \\ \operatorname{sen} x \cdot \operatorname{sen} y, & \text { si } x \neq y\end{cases}
$$

es una métrica fuzzy para el producto usual sobre $X=] 0, \pi[$.

En la Sección 2.8 las métricas fuzzy que nos encontramos se definen a partir de otras métricas fuzzy. Así, por ejemplo, obtenemos la métrica $(M, \cdot)$ de la Proposición 2.8.5, dada por

$$
M(x, y, t)=\frac{h(t)}{h(t)+1-N(x, y, t)}
$$

donde $h: \mathbb{R}^{+} \rightarrow \mathbb{R}^{+}$es una función continua y creciente y $N$ una métrica fuzzy para el producto.

En la Sección 2.9, las métricas fuzzy están definidas atendiendo a una partición de $X$. El valor de la función $M$ depende de la localización de los puntos en la partición hecha sobre el conjunto $X$. Podemos ver una métrica de éstas en la Proposición 2.9.1 donde la métrica $(M, *)$ sobre $X$ viene dada por

$$
M(x, y, t)= \begin{cases}N(x, y, t), & \text { si } x, y \in A \text { ó } x, y \in B \\ N(x, y, t) * \varphi(t), & \text { en otro caso }\end{cases}
$$

donde $\left.\left.\varphi: \mathbb{R}^{+} \rightarrow\right] 0,1\right]$ es una función continua y creciente, $(N, *)$ una métrica fuzzy sobre $X$ y $X=A \cup B$, con $A \cap B=\phi$.

Finalmente, en la Sección 2.10 vemos cómo extender una métrica fuzzy de dos formas distintas. La primera forma, que podemos ver en la Proposición 2.10.1, permite extender a $H \cup K$, donde $H$ y $K$ son conjuntos no disjuntos, y en los que están definidas dos métricas fuzzy $M_{H}$ y $M_{K}$, respectivamente, coincidentes en $H \cap K$, una métrica fuzzy $M$ que coincide con $M_{H}$ en $H$ 
y con $M_{K}$ en $K$, es decir, $\left.M\right|_{H}=M_{H}$ y $\left.M\right|_{K}=M_{K}$. Como aplicación a este método, tenemos los Ejemplos 2.10.2 y 2.10.3. La segunda forma, está basada en el concepto de isometría (fuzzy), que nos permite transportar la métrica fuzzy de un espacio a otro que estaba desprovisto de métrica fuzzy (Proposición 2.10.4). Como aplicación, tenemos que

$$
N(x, y)=\frac{\min \left\{e^{x}, e^{y}\right\}}{\max \left\{e^{x}, e^{y}\right\}}
$$

es una métrica fuzzy estacionaria sobre $\mathbb{R}$, al considerar como t-norma el producto usual, y la topología $\tau_{N}$ es la usual de $\mathbb{R}$.

En este capítulo, $X$ denotará un conjunto no vacío y $M$ un conjunto fuzzy de $X \times X \times \mathbb{R}^{+}$.

\subsection{Generalización de métricas fuzzy conocidas}

Proposición 2.2.1. Sea $f: X \rightarrow \mathbb{R}^{+}$una función inyectiva y sea $g: \mathbb{R}^{+} \rightarrow$ $[0,+\infty[$ una función continua y creciente. Fijados $\alpha, \beta>0$, definimos $M$ por

$$
M(x, y, t)=\left(\frac{(\min \{f(x), f(y)\})^{\alpha}+g(t)}{(\max \{f(x), f(y)\})^{\alpha}+g(t)}\right)^{\beta}
$$

Entonces $(M, \cdot)$ es una métrica fuzzy sobre $X$.

\section{Demostración.}

$(G V 1)$ Es obvio que $M(x, y, t)>0$ para todo $x, y \in X, t>0$.

$(G V 2)$ Veamos que $M(x, y, t)=1 \Leftrightarrow x=y$. En efecto:

$$
\left(\frac{(\min \{f(x), f(y)\})^{\alpha}+g(t)}{(\max \{f(x), f(y)\})^{\alpha}+g(t)}\right)^{\beta}=1 \Leftrightarrow
$$


$\Leftrightarrow \min \{f(x), f(y)\}=\max \{f(x), f(y)\} \Leftrightarrow f(x)=f(y) \Leftrightarrow x=y$

$(G V 3)$ Es obvio que $M(x, y, t)=M(y, x, t)$.

$(G V 4)$ Veamos que se verifica $M(x, y, t) \cdot M(y, z, s) \leq M(x, z, t+s)$

Supongamos que $f(x) \leq f(z)$. Podemos distinguir tres casos:

(1) $f(x) \leq f(y) \leq f(z)$

(2) $f(y) \leq f(x) \leq f(z)$

(3) $f(x) \leq f(z) \leq f(y)$

Pues bien, si ponemos

$$
M(x, z, t+s)=\left(\frac{f(x)^{\alpha}+g(t+s)}{f(y)^{\alpha}+g(t+s)}\right)^{\beta} \cdot\left(\frac{f(y)^{\alpha}+g(t+s)}{f(z)^{\alpha}+g(t+s)}\right)^{\beta}
$$

es fácil ver que en los tres casos se cumple la desigualdad

$$
M(x, z, t+s) \geq M(x, y, t) \cdot M(y, z, s)
$$

ya que g es creciente. En efecto:

(1)

$$
\begin{aligned}
& M(x, z, t+s)=\left(\frac{f(x)^{\alpha}+g(t+s)}{f(y)^{\alpha}+g(t+s)}\right)^{\beta} \cdot\left(\frac{f(y)^{\alpha}+g(t+s)}{f(z)^{\alpha}+g(t+s)}\right)^{\beta} \geq \\
& \geq\left(\frac{f(x)^{\alpha}+g(t)}{f(y)^{\alpha}+g(t)}\right)^{\beta} \cdot\left(\frac{f(y)^{\alpha}+g(s)}{f(z)^{\alpha}+g(s)}\right)^{\beta}=M(x, y, t) \cdot M(y, z, s)
\end{aligned}
$$


(2)

$$
\begin{gathered}
M(x, z, t+s)=\left(\frac{f(x)^{\alpha}+g(t+s)}{f(y)^{\alpha}+g(t+s)}\right)^{\beta} \cdot\left(\frac{f(y)^{\alpha}+g(t+s)}{f(z)^{\alpha}+g(t+s)}\right)^{\beta} \geq \\
\geq\left(\frac{f(y)^{\alpha}+g(t+s)}{f(z)^{\alpha}+g(t+s)}\right)^{\beta} \geq\left(\frac{f(y)^{\alpha}+g(t)}{f(x)^{\alpha}+g(t)}\right)^{\beta} \cdot\left(\frac{f(y)^{\alpha}+g(s)}{f(z)^{\alpha}+g(s)}\right)^{\beta}= \\
=M(x, y, t) \cdot M(y, z, s)
\end{gathered}
$$

(3)

$$
\begin{gathered}
M(x, z, t+s)=\left(\frac{f(x)^{\alpha}+g(t+s)}{f(y)^{\alpha}+g(t+s)}\right)^{\beta} \cdot\left(\frac{f(y)^{\alpha}+g(t+s)}{f(z)^{\alpha}+g(t+s)}\right)^{\beta} \geq \\
\geq\left(\frac{f(x)^{\alpha}+g(t+s)}{f(y)^{\alpha}+g(t+s)}\right)^{\beta} \geq\left(\frac{f(x)^{\alpha}+g(t)}{f(y)^{\alpha}+g(t)}\right)^{\beta} \cdot\left(\frac{f(z)^{\alpha}+g(s)}{f(y)^{\alpha}+g(s)}\right)^{\beta} \\
=M(x, y, t) \cdot M(y, z, s)
\end{gathered}
$$

La prueba es similar en el caso $f(x)>f(z)$.

Nota 2.2.2. Es fácil demostrar que para $r \in] 0,1[y t>0$, las bolas abiertas vienen dadas por

$$
\begin{gathered}
B(x, r, t)=\left\{y \in X:\left(\left(f^{\alpha}(x)+g(t)\right)(1-r)^{\frac{1}{\beta}}-g(t)\right)^{\frac{1}{\alpha}} \leq\right. \\
\left.f(y) \leq\left(\frac{f^{\alpha}(x)+g(t)}{(1-r)^{\frac{1}{\beta}}}-g(t)\right)^{\frac{1}{\alpha}}\right\}
\end{gathered}
$$


Los siguientes tres ejemplos son casos particulares de la anterior proposición.

(A) Si tomamos en (2.1) $f(x)=x, g(t)=t, \alpha=1$ y $\beta=1$, entonces como caso particular se tiene que

$$
M(x, y, t)=\frac{\min \{x, y\}+t}{\max \{x, y\}+t}
$$

es una métrica fuzzy sobre $X=\mathbb{R}^{+}$.

Es fácil demostrar que la topología sobre $X$ deducida de $M$ coincide con la topología usual de $\mathbb{R}$ restringida a $\mathbb{R}^{+}$. En efecto:

$$
B(x, r, t)=\{y \in X: M(x, y, t)>1-r\}=] x-r(x+t), \frac{x+r t}{1-r}[
$$

ya que:

Si $x \leq y$,

$$
\begin{gathered}
M(x, y, t)=\frac{x+t}{y+t}>1-r \Leftrightarrow x+t>(1-r)(y+t) \Leftrightarrow \\
\Leftrightarrow y<\frac{x+t}{1-r}-t=\frac{x+r t}{1-r}
\end{gathered}
$$

Si $y \leq x$,

$$
M(x, y, t)=\frac{y+t}{x+t}>1-r \Leftrightarrow y+t>(1-r)(x+t) \Leftrightarrow y>x-r(x+t)
$$

Luego las bolas son intervalos abiertos que pueden hacerse tan pequeños como se desee y por lo tanto la topología asociada a esta métrica es la topología usual dado que para cualquier $x \in X$ y $\delta>0$, el intervalo de la forma $] x-\delta, x+\delta$ [ contiene alguna bola abierta $B(x, r, t)$, y recíprocamente, 
toda bola $B(x, r, t)$ con $r \in] 0,1[$ y $t>0$, contiene algún intervalo de la forma ]$x-\delta, x+\delta[$.

(B) Tomamos $X=\mathbb{N}$ y elegimos en (2.1) $f(x)=x, x \in X, g(t)=0$, $t>0, \alpha=\beta=1$. Entonces se obtiene la métrica fuzzy estacionaria $(M, \cdot)$ sobre $X,[14]$, donde $M$ viene dada por

$$
M(x, y)=\frac{\min \{x, y\}}{\max \{x, y\}}
$$

Observar que el elemento más cercano, respecto de $M$, a $n \in X$, es siempre $n+1$.

En este caso, la topología $\tau_{M}$ es la topología discreta, ya que para cada $n \in X$, si tomamos $0<r<1-\frac{n}{n+1}$, se tiene que $B(n, r, t)=\{n\}$.

(C) Tomamos $X=]-k,+\infty[(k>0)$, y elegimos en (2.1) $f(x)=x$, $x \in X, g(t)=k$, para todo $t>0$, siendo $k>0$ y $\alpha=\beta=1$. Entonces se obtiene la métrica fuzzy estacionaria $(M, \cdot)$ sobre $X,[40]$, donde $M$ viene dada por

$$
M(x, y)=\frac{\min \{x, y\}+k}{\max \{x, y\}+k}
$$

Obsérvese que $(M, \wedge)$ no es una métrica fuzzy. En efecto, basta tomar $x=0$, $y=10$ y $z=1$ y se tiene

$$
M(x, z)=\frac{k}{10+k}<\min \left\{\frac{k}{1+k}, \frac{1+k}{10+k}\right\}=\min \{M(x, y), M(y, z)\} .
$$

Veamos la topología en $X$ que se deduce de $M$.

$$
\begin{gathered}
B(x, r, t)=\{y \in X, y<x: M(x, y)>1-r\} \cup \\
\cup\{y \in X, y>x: M(x, y)>1-r\}=
\end{gathered}
$$




$$
\begin{aligned}
=\{y \in X: y> & x-r(x+k)\} \cup\left\{y \in X: y<\frac{x-k r}{1-r}\right\}= \\
= & ] x-r(x+k), \frac{x+k r}{1-r}[
\end{aligned}
$$

Vemos que las bolas son intervalos abiertos que pueden hacerse tan pequeños como se desee y, por lo tanto la topología, como en el caso (A), coincide con la topología usual de $\mathbb{R}$ restringida a $]-k,+\infty[$.

\subsection{Métricas fuzzy definidas mediante métricas}

En los siguientes dos ejemplos $g: \mathbb{R}^{+} \rightarrow \mathbb{R}^{+}$será una función continua y creciente y $d$ será una métrica sobre $X$.

Proposición 2.3.1. Sea $m \in \mathbb{R}^{+}$. Definimos la función $M$ por

$$
M(x, y, t)=\frac{g(t)}{g(t)+m \cdot d(x, y)}
$$

Entonces $(M, \cdot)$ es una métrica fuzzy sobre $X$.

\section{Demostración.}

Las propiedades $(G V 1)-(G V 3)$ y $(G V 5)$ son evidentes.

Veamos (GV4). Sean $x, y, z \in X, t>0$. Veamos que

$$
M(x, z, t+s) \geq M(x, y, t) \cdot M(y, z, s) .
$$

Para ello vamos a ver que

(1) $M(x, z, t) \geq M(x, y, t) \cdot M(y, z, t)$ y que 
(2) $M$ es creciente.

Para ver (1) hemos de probar que

$$
\frac{g(t)}{g(t)+m \cdot d(x, z)} \geq \frac{g(t)}{g(t)+m \cdot d(x, y)} \cdot \frac{g(t)}{g(t)+m \cdot d(y, z)}
$$

que es lo mismo que probar que

$$
(g(t)+m \cdot d(x, y)) \cdot(g(t)+m \cdot d(y, x)) \geq(g(t)+m \cdot d(x, z)) \cdot g(t) .
$$

Pues bien

$$
\begin{gathered}
(g(t)+m \cdot d(x, y)) \cdot(g(t)+m \cdot d(y, x))= \\
=g(t)^{2}+g(t) \cdot m \cdot d(x, y)+g(t) \cdot m \cdot d(y, z)+m^{2} \cdot d(x, y) \cdot d(y, z)= \\
=g(t)^{2}+g(t) \cdot m(d(x, y)+d(y, z))+m^{2} \cdot d(x, y) \cdot d(y, z) \geq \\
\geq g(t)^{2}+g(t) \cdot m \cdot d(x, z)+m^{2} \cdot d(x, y) \cdot d(y, z) \geq \\
\geq g(t)^{2}+g(t) m \cdot d(x, z)=g(t) \cdot(g(t)+m d(x, z))
\end{gathered}
$$

Para probar (2), sean $x, y \in X$ y $t, s>0$. Veamos que

$$
M(x, y, t+s) \geq M(x, y, t) .
$$

Es evidente que

$$
g(t+s) \cdot m \cdot d(x, y) \geq g(t) \cdot m \cdot d(x, y)
$$

pues $g$ es creciente. Por lo tanto

$$
\begin{gathered}
g(t) \cdot g(t+s)+g(t+s) \cdot m \cdot d(x, y) \geq g(t) \cdot g(t+s)+g(t) m \cdot d(x, y) \Rightarrow \\
\Rightarrow(g(t)+m \cdot d(x, y)) g(t+s) \geq g(t)(g(t+s)+m \cdot d(x, y)) \Rightarrow \\
\Rightarrow \frac{g(t+s)}{g(t+s)+m \cdot d(x, y)} \geq \frac{g(t)}{g(t)+m \cdot d(x, y)}
\end{gathered}
$$


Así, de (1) y (2) se tiene que para $x, y, x \in X, t, s>0$

$$
M(x, z, t+s) \geq M(x, y, t+s) \cdot M(y, z, t+s) \geq M(x, y, t) \cdot M(y, z, s)
$$

Como caso particular, si elegimos $g(t)=t^{n}$, con $n \in \mathbb{N}$ y $m=1$, entonces obtenemos

$$
M(x, y, t)=\frac{t^{n}}{t^{n}+d(x, y)},
$$

y así $(M, \wedge)$ es una métrica fuzzy sobre $X$ como podemos ver en [49]. En particular, para $n=1$, tenemos

$$
M(x, y, t)=\frac{t}{t+d(x, y)}
$$

conocida como la métrica fuzzy estándar. La topología sobre $X$ deducida de la métrica fuzzy estándar coincide con la topología asociada a la métrica $d$, es decir, $\tau_{M}=\tau(d)$.

Por otra parte, si en la ecuación (2.4) $g(t)$ es una función constante, es decir, $g(t)=k>0$, y $m=1$, obtenemos

$$
M(x, y, t)=\frac{k}{k+d(x, y)}
$$

y así $(M, \cdot)$ es una métrica fuzzy estacionaria sobre $X$ pero, en general $(M, \wedge)$ no lo es. Para verificarlo, basta tomar $X=\mathbb{R}, d(x, y)=|x-y|, x=1$, $y=10$ y $z=100$. Así tenemos que $M(x, z)<\min \{M(x, y), M(y, z)\}$ en contradicción con (GV4).

Proposición 2.3.2. Definimos la función $M$ por

$$
M(x, y, t)=e^{-\frac{d(x, y)}{g(t)}}
$$

Entonces $(M, \cdot)$ es una métrica fuzzy sobre $X$. 


\section{Demostración.}

Las propiedades $(G V 1)-(G V 3)$ y $(G V 5)$ son evidentes.

Veamos $(G V 4)$. Sean $x, y, z \in X$. Veamos que

$$
M(x, z, t+s) \geq M(x, y, t) \cdot M(y, z, s) .
$$

Por la desigualdad triangular de $d$ tenemos que $d(x, z) \leq d(x, y)+d(y, z)$. Luego

$$
d(x, z) \leq \frac{g(t+s)}{g(t)} d(x, y)+\frac{g(t+s)}{g(s)} d(y, z)
$$

(ya que $\frac{g(t+s)}{g(t)} \geq 1$ pues $g$ es creciente), por tanto

$$
\frac{d(x, z)}{g(t+s)} \leq \frac{d(x, y)}{g(t)}+\frac{d(y, z)}{g(s)}
$$

en consecuencia

$$
e^{\frac{d(x, z)}{g(t+s)}} \leq e^{\frac{d(x, y)}{g(t)}} \cdot e^{\frac{d(y, z)}{g(s)}}
$$

y por lo tanto

$$
e^{-\frac{d(x, z)}{g(t+s)}} \geq e^{-\frac{d(x, y)}{g(t)}} \cdot e^{-\frac{d(y, z)}{g(s)}}
$$

y así tenemos probado que $M(x, z, t+s) \geq M(x, y, t) \cdot M(y, z, s)$.

Nota 2.3.3. En este caso, para cada $x \in X, r \in] 0,1[y t>0$, es fácil demostrar que $B(x, r, t)=B_{R}(x)$ donde $R=g(t) \ln \frac{1}{1-r}$. Como consecuencia, para cada $x \in X$ y $R>0$ tenemos que $B_{R}(x)=B(x, r, t)$ donde $r=1-\frac{1}{e^{\frac{R}{g(t)}}}$ para todo $t>0$ y asi $\tau_{M}$ coincide con $\tau(d)$.

Ejemplo 2.3.4. Como caso particular de la proposición anterior, si tomamos $g(t)=t$ en (2.6), obtenemos la función $M$ dada por

$$
M(x, y, t)=e^{-\frac{d(x, y)}{t}}
$$

En este caso, $(M, \wedge)$ es una métrica fuzzy sobre $X$ que podemos ver en [14]. 
Ejemplo 2.3.5. Por otro lado, si tomamos en (2.6) la función constante $g(t)=k>0$, tenemos

$$
M(x, y, t)=e^{\frac{-d(x, y)}{k}}
$$

En este caso, $(M, \cdot)$ es una métrica fuzzy sobre $X$, pero en general, $(M, \wedge)$ no lo es.

En efecto, tomemos $X=\mathbb{R}, k=1, d(x, y)=|x-y| y x=0, y=\frac{1}{2} y$ $z=1$. Se tiene entonces:

$$
\frac{1}{e^{d(x, z)}}<\min \left\{\frac{1}{e^{d(x, y)}}, \frac{1}{e^{d(y, z)}}\right\} \text { ya que } \frac{1}{e}<\min \left\{\frac{1}{e^{\frac{1}{2}}}, \frac{1}{e^{\frac{1}{2}}}\right\} \text { pues } e>e^{\frac{1}{2}} .
$$

Proposición 2.3.6. Sea $(X, d)$ un espacio métrico tal que $d(x, y)<k$, $\forall x, y \in X$. Sea $\left.g: \mathbb{R}^{+} \rightarrow\right] k,+\infty[$ una función continua y creciente. Definimos la función $M$ por

$$
M(x, y, t)=1-\frac{d(x, y)}{g(t)}
$$

Entonces $(M, \mathfrak{L})$ es una métrica fuzzy sobre $X$.

Demostración.

$(G V 1)$ Veamos que $M(x, y, t)>0$. En efecto, como $g(t)>k$ y $d(x, y)<k$ entonces

$$
M(x, y, t)=1-\frac{d(x, y)}{g(t)}>0
$$

pues $d(x, y)<k$ y $g(t) \in] k,+\infty[$.

$(G V 2)$ Veamos que $M(x, y, t)=1 \Leftrightarrow x=y$. En efecto:

$$
M(x, y, t)=1 \Leftrightarrow 1-\frac{d(x, y)}{g(t)}=1 \Leftrightarrow \frac{d(x, y)}{g(t)}=0 \Leftrightarrow d(x, y)=0 \Leftrightarrow x=y
$$

$(G V 3)$ Obviamente $M(x, y, t)=M(y, x, t) \forall x, y \in X$. 
$(G V 4)$ Veamos que se verifica que

$$
M(x, z, t+s) \geq \max \{0, M(x, y, t)+M(y, z, s)-1\} .
$$

Como $d(x, z) \leq d(x, y)+d(y, z)$, entonces

$$
\frac{d(x, z)}{g(t+s)} \leq \frac{d(x, y)}{g(t+s)}+\frac{d(y, z)}{g(t+s)}
$$

y como $g(t+s)>g(t)$ y $g(t+s)>g(s)$, pues $g$ es creciente, entonces

$$
\begin{aligned}
\frac{d(x, z)}{g(t+s)} & \leq \frac{d(x, y)}{g(t)}+\frac{d(y, z)}{g(s)} \Rightarrow \frac{-d(x, z)}{g(t+s)} \geq \frac{-d(x, y)}{g(t)}-\frac{d(y, z)}{g(s)} \Rightarrow \\
& \Rightarrow 1-\frac{d(x, z)}{g(t+s)} \geq 1-\frac{d(x, y)}{g(t)}+1-\frac{d(y, z)}{g(s)}-1
\end{aligned}
$$

y así tenemos que $M(x, z, t+s) \geq \max \{0, M(x, y, t)+M(y, z, s)-1\}$

$(G V 5) M(x, y,-):] 0,+\infty[\rightarrow[0,1]$ es obviamente continua pues $g$ es continua.

Nota 2.3.7. En este caso para cada $x \in X, r \in] 0,1[y t>0$ tenemos que $B(x, r, t)=B_{r \cdot g(t)}(x)$.

Como consecuencia, para cada $x \in X$ y $R>0$ tenemos que $B_{R}(x)=$ $B(x, r, t)$ donde $r=\frac{R}{g(t)}$ para todo $t>0$ y asi $\tau_{M}$ coincide con $\tau(d)$.

Ejemplo 2.3.8. Si ahora en (2.7) tomamos g como una función constante $g(t)=K>k$, entonces obtenemos la función $M$ dada por

$$
M(x, y)=1-\frac{d(x, y)}{K}
$$


y en este caso, $(M, \mathfrak{L})$ es una métrica fuzzy estacionaria sobre $X$, pero $(M, \cdot)$ en general no lo es.

Para ver que en este último caso $(M, \cdot)$ no es una métrica fuzzy, basta tomar $X=[0,10], d(x, y)=|x-y|$ y $x=0, y=1 y z=2$. Asi tenemos que

$$
\begin{gathered}
M(x, z)=1-\frac{2}{K} \leq M(x, y) \cdot M(y, z)= \\
=\left(1-\frac{1}{K}\right) \cdot\left(1-\frac{1}{K}\right)=\left(1-\frac{1}{K}\right)^{2}=1-\frac{2}{K}+\frac{1}{K^{2}} .
\end{gathered}
$$

\subsection{La métrica fuzzy discreta}

Proposición 2.4.1. Sea $\left.\varphi: \mathbb{R}^{+} \rightarrow\right] 0,1[$ una función continua y creciente.

Definimos sobre un conjunto $X$ la función $M$ por

$$
M(x, y, t)= \begin{cases}1, & \text { si } x=y \\ \varphi(t), & \text { si } x \neq y\end{cases}
$$

Entonces $(M, \wedge)$ es una métrica fuzzy sobre $X$.

\section{Demostración.}

Las propiedades $(G V 1)-(G V 3)$ y $(G V 5)$ son evidentes.

Veamos $(G V 4)$. Sean $x, y, z \in X$. Veamos que

$$
M(x, z, t+s) \geq \min \{M(x, y, t), M(y, z, s)\} .
$$

Si $x=z \neq y$ entonces obviamente

$$
M(x, z, t+s)=1 \geq \min \{\varphi(t), \varphi(s)\}=M(x, y, t) \wedge M(y, z, s) .
$$


Si $x \neq z$ basta observar que

$$
\varphi(t+s) \geq \varphi(t) \text { y que } \varphi(t+s) \geq \varphi(s), \text { pues } \varphi \text { es creciente } .
$$

En particular, si $\varphi$ es constante, $\varphi(t)=k \in] 0,1[$, obtenemos la métrica fuzzy estacionaria

$$
M(x, y, t)= \begin{cases}1, & \text { si } x=y \\ k, & \text { si } x \neq y\end{cases}
$$

que llamaremos la métrica fuzzy discreta. Su nombre viene justificado porque efectivamente la topología asociada a dicha métrica es la topología discreta. Para ello, basta observar que $\{x\}$ es abierto en $\left(X, \tau_{M}\right)$ para cualquier $x \in X$. En efecto, $B(x, 1-\varphi(t), t)=\{x\}$

\subsection{Métricas fuzzy definidas por funciones simétri-} cas

Proposición 2.5.1. Sea $F: X \times X \rightarrow] 0, \frac{1}{2}[$ una función simétrica, es decir, $F(x, y)=F(y, x), \forall x, y \in X$. Definimos la función $M$ por

$$
M(x, y)= \begin{cases}1, & \text { si } x=y \\ F(x, y), & \text { si } x \neq y\end{cases}
$$

Entonces $(M, \mathfrak{L})$ es una métrica fuzzy estacionaria sobre $X$.

\section{Demostración.}

La propiedades $(G V 1)-(G V 3)$ y $(G V 5)$ son evidentes. 
Veamos $(G V 4)$. Sean $x, y, z \in X$. Veamos que se verifica que

$$
M(x, z) \geq \max \{0, M(x, y)+M(y, z)-1\}
$$

Si un par de elementos son iguales, la desigualdad es obvia.

Supongamos que los elementos $x, y, z$ son los tres distintos dos a dos. Entonces:

$$
M(x, y)+M(y, z)=F(x, y)+F(y, z)<\frac{1}{2}+\frac{1}{2}=1
$$

Así queda probada en cualquier caso la desigualdad triangular.

Como aplicación, consideremos la función $F(x, y)=\cos ^{2} x-\operatorname{sen}^{2} y$.

Veamos que $F(x, y)=\cos ^{2} x-\operatorname{sen}^{2} y$ es simétrica. Se tiene que

$$
F(x, y)-F(y, x)=\cos ^{2} x-\operatorname{sen}^{2} y-\cos ^{2} y+\operatorname{sen}^{2} x=0
$$

y por lo tanto $F(x, y)=F(y, x)$.

Obviamente $\left.0<F(x, y)<\frac{1}{2}, \forall x, y \in\right] 0, \frac{\pi}{4}[$.

Luego $F$ cumple en ]0, $\frac{\pi}{4}[$ las condiciones de la proposición anterior.

Entonces, si tomamos $X=] 0, \frac{\pi}{4}\left[\right.$ y $F(x, y)=\cos ^{2} x-\operatorname{sen}^{2} y$, tenemos que (2.9) se convierte en

$$
M(x, y)= \begin{cases}1, & \text { si } x=y \\ \cos ^{2} x-\operatorname{sen}^{2} y, & \text { si } x \neq y\end{cases}
$$

y $(M, \mathfrak{L})$ es una métrica fuzzy estacionaria sobre $X$ pero $(M, \cdot)$ no lo es. En efecto, basta tomar $x=\frac{11 \pi}{45}, y=\frac{\pi}{180}$ y $z=\frac{43 \pi}{180}$, y se tiene que

$$
M(x, z) \cong 0,052
$$


mientras que

$$
M(x, y) \cdot M(y, z) \cong 0,516 \cdot 0,534 \cong 0,27 .
$$

Proposición 2.5.2. Sea $f: Y \rightarrow] 0, \frac{1}{2}[$ una función y $X$ un conjunto de números reales tales que $x+y \in Y, \forall x, y \in X$. Definimos la función $M$ por

$$
M(x, y)= \begin{cases}1, & \text { si } x=y \\ f(x+y), & \text { si } x \neq y\end{cases}
$$

Entonces $(M, \mathfrak{L})$ es una métrica fuzzy estacionaria sobre $X$. (Obsérvese que $F(x, y)=f(x+y)$ es una función simétrica).

\section{Demostración.}

Las propiedades $(G V 1)-(G V 3)$ y $(G V 5)$ son evidentes.

Veamos $(G V 4)$. Sean $x, y, z \in X$. Tenemos que ver que

$$
M(x, z) \geq \max \{0, M(x, y)+M(y, z)-1\}
$$

y supondremos que $M(x, y)+M(y, z)>1$, pues de lo contrario la desigualdad es obvia.

Si $x=z \neq y$, es evidente.

Si $x=y \neq z$, entonces

$M(x, z)=f(x+z)=1+f(x+z)-1=1+f(y, z)-1=M(x, y)+M(y, z)-1$

Si $x \neq y \neq z \neq z$, entonces

$$
M(x, y)+M(y, z)-1=f(x+y)+f(y+z)-1<
$$




$$
<\frac{1}{2}+\frac{1}{2}-1=1-1=0<f(x+z)=M(x, z)
$$

Como caso particular, si tomamos $X=] \frac{\pi}{6}, \frac{\pi}{4}[$ y $f(x)=\cos x$, obtenemos que $f(x) \in] 0, \frac{1}{2}[$. Definimos

$$
M(x, y)= \begin{cases}1, & \text { si } x=y \\ \cos (x+y), & \text { si } x \neq y\end{cases}
$$

y según la proposición anterior, $(M, \mathfrak{L})$ es una métrica fuzzy sobre $X$ pero $(M, \cdot)$ no lo es. En efecto, basta tomar $x=\frac{11 \pi}{45}, y=\frac{31 \pi}{180} \mathrm{y} z=\frac{43 \pi}{180}$, y tenemos que

$$
M(x, y) \cdot M(y, z) \cong 0,258 \cdot 0,275=0,07
$$

mientras que

$$
M(x, z) \cong 0,052<0,07=M(x, y) \cdot M(y, z) .
$$

Proposición 2.5.3. Sea $f: Y \rightarrow] 0, \frac{1}{2}[$ una función y $X$ un conjunto de números reales tales que $x-y \in Y$. Supongamos que $f(z)=f(-z), \forall z \in Y$. Definimos la función $M$ por

$$
M(x, y)= \begin{cases}1, & \text { si } x=y \\ f(x-y), & \text { si } x \neq y\end{cases}
$$

Entonces $(M, \mathfrak{L})$ es una métrica fuzzy estacionaria sobre $X$. (Obsérvese que $F(x, y)=f(x-y)$ es una función simétrica).

La demostración es análoga a la anterior.

Como caso particular, si tomamos $X=] 0, \frac{\sqrt{2}}{4}\left[\right.$ y $f(x)=x^{2}$, tenemos

$$
M(x, y)= \begin{cases}1, & \text { si } x=y \\ (x-y)^{2}, & \text { si } x \neq y\end{cases}
$$


Entonces $(M, \mathfrak{L})$ es una métrica fuzzy estacionaria sobre $X$, pero $(M, \cdot)$ no lo es. En efecto, para ver que $M$ cumple las condiciones de la proposición, tenemos que ver que

$$
\left.0<(x-y)^{2}<\frac{1}{2}, \forall x, y \in\right] 0, \frac{\sqrt{2}}{4}[
$$

En efecto, si $x, y \in] 0, \frac{\sqrt{2}}{4}\left[\right.$ entonces $-\frac{\sqrt{2}}{4}<x-y<\frac{\sqrt{2}}{4}$, luego $(x-y)^{2}<$ $\left(\frac{\sqrt{2}}{4}\right)^{2}=\frac{2}{16}=\frac{1}{8}$.

Además $f(x)=x^{2}=f(-x)$ y $F(x, y)=f(x-y)$ es una función simétrica.

Por otro lado, para ver que $(M, \cdot)$ no es una métrica fuzzy, basta tomar $x=0,01, y=0,3$ y $z=0,001$. De esta forma tenemos que

$$
\begin{gathered}
M(x, z)=(x-z)^{2}=0,009^{2}=0,000081 \\
M(x, y) \cdot M(y, z)=(x-y)^{2} \cdot(y-z)^{2}=0,0841 \cdot 0,089401 \cong 0,00751
\end{gathered}
$$

Luego

$$
M(x, z)<M(x, y) \cdot M(y, z) .
$$

Proposición 2.5.4. Sea $f: X \rightarrow] 0, \frac{1}{2}[$ una función y definimos $M$ por

$$
M(x, y)= \begin{cases}1, & \text { si } x=y \\ f(x)+f(y), & \text { si } x \neq y\end{cases}
$$

Entonces $(M, \mathfrak{L})$ es una métrica fuzzy estacionaria sobre $X$. Obsérvese que $F(x, y)=f(x)+f(y)$ es una función simétrica y que ahora la imagen de $F$ es $] 0,1[$, es decir, $F: X \times X \rightarrow] 0,1[$.

\section{Demostración.}

Las propiedades $(G V 1)-(G V 3)$ y $(G V 5)$ son evidentes. 
Veamos (GV4). Sean $x, y, z \in X$. Veamos que

$$
M(x, z) \geq \max \{0, M(x, y)+M(y, z)-1\} .
$$

Supondremos que $M(x, y)+M(y, z)>1$, pues de lo contrario la desigualdad es obvia.

Si $x \neq y \neq z \neq x$,

$$
\begin{gathered}
M(x, z) \geq M(x, y)+M(y, z)-1 \Leftrightarrow \\
\Leftrightarrow f(x)+f(z) \geq f(x)+f(y)+f(y)+f(z)-1 \Leftrightarrow 0 \geq 2 f(y)-1
\end{gathered}
$$

Pero se tiene que

$$
\begin{aligned}
& 2 f(y)-1<2 \cdot \frac{1}{2}-1=1-1=0 \Rightarrow \\
& \Rightarrow M(x, z) \geq M(x, y)+M(y, z)-1
\end{aligned}
$$

Si $x=z \neq y$, la desigualdad es obvia.

Si $x=y \neq z$, ó si $y=z \neq x$, se da claramente la igualdad.

Ejemplo 2.5.5. Como caso particular de la Proposición 2.5.4, si $X=$ ] $2,+\infty\left[\right.$ y tomamos $f(x)=\frac{1}{x}$, tenemos

$$
M(x, y)= \begin{cases}1, & \text { si } x=y \\ \frac{1}{x}+\frac{1}{y}, & \text { si } x \neq y\end{cases}
$$

Entonces $(M, \mathfrak{L})$ es una métrica fuzzy estacionaria sobre $X$, pero $(M, \cdot)$ no lo es.

Para ver que $(M, \cdot)$ no es una métrica fuzzy, basta tomar $x=1000, y=3$ $y z=10000$, y así

$$
M(x, z)=\left(\frac{1}{1000}+\frac{1}{10000}\right)=1,1 \cdot 10^{-3}<
$$




$$
<\left(\frac{1}{1000}+\frac{1}{3}\right) \cdot\left(\frac{1}{3}+\frac{1}{10000}\right)=M(x, y) \cdot M(y, z)
$$

Además, si $x \in X$ y tomamos $r<\frac{1}{2}-\frac{1}{x}$, entonces $B(x, r, t)=\{x\}$ y por lo tanto $\tau_{M}$ es la topología discreta.

Ejemplo 2.5.6. Como caso particular de la Proposición 2.5.4, si $X=] 0, \frac{1}{2}[$ y tomamos $f$ como la función identidad sobre $X$, tenemos

$$
M(x, y)= \begin{cases}1, & \text { si } x=y \\ x+y, & \text { si } x \neq y\end{cases}
$$

Entonces $(M, \mathfrak{L})$ es una métrica fuzzy sobre $X$, pero $(M, \cdot)$ no lo es.

Para ver que $(X, M, \cdot)$ no es un espacio métrico fuzzy, basta tomar $x=$ $0,001, y=0,4 y z=0,0001$, y entonces

$M(x, z)=x+z=0,0011<0,1604=(x+y) \cdot(y+z)=M(x, y) \cdot M(y, z)$.

Además $B(x, r, t)=\{y \in X: x+y>1-r\}$. Si tomamos $0<r<\frac{1}{2}-x$, entonces $B(x, r, t)=\{x\}$ y por lo tanto $\tau_{M}$ es la topología discreta.

\subsection{Métricas fuzzy definidas a partir de un par de funciones}

Proposición 2.6.1. Sean $g, h: X \rightarrow] 0,1[$ dos funciones que cumplen que $\sup \{g(x): x \in X\}<\inf \{h(x): x \in X\}$ y $(h+g)(x)=c$. Definimos la función $M$ por

$$
M(x, y)= \begin{cases}1, & \text { si } x=y \\ h(x)-g(y), & \text { si } x \neq y\end{cases}
$$

Entonces $(M, \mathfrak{L})$ es una métrica fuzzy estacionaria sobre $X$. 


\section{Demostración.}

$(G V 1) M(x, y)>0$ por definición de $g$ y $h$.

$(G V 2) M(x, y)=1 \Leftrightarrow x=y$, es obvio por definición de $M$.

$(G V 3) M(x, y)=h(x)-g(y)=c-g(x)-g(y)=$

$=(c-g(y))-g(x)=h(y)-g(x)=M(y, x)$.

(GV4) Sean $x, y, z \in X$. Veamos que

$$
M(x, z) \geq M(x, y) * M(y, z)=\max \{0, M(x, y)+M(y, z)-1\}
$$

Supondremos que $M(x, y)+M(y, z)>1$ pues de lo contrario la desigualdad es obvia.

Si $x=z$, la desigualdad es obvia.

Si $x \neq y \neq z \neq x$, entonces se tiene:

$$
\begin{gathered}
M(x, z)=h(x)-g(z)=h(x)-g(y)+g(y)-g(z)= \\
=h(x)-g(y)+(c-h(y))-g(z) \geq \\
\geq h(x)-g(y)+h(y)-g(z)-1=M(x, y) \mathfrak{L} M(y, z) .
\end{gathered}
$$

Además, como las funciones $h$ y $g$ satisfacen que $(h+g)(x)=c$, con $0<c<2, \forall x \in X$, entonces $M$ se puede escribir como

$$
M(x, y)= \begin{cases}1, & \text { si } x=y \\ h(x)+h(y)-c, & \text { si } x \neq y\end{cases}
$$


Esta última métrica fuzzy puede considerarse como un caso particular de la Proposición 2.5.4 cuando $c<1$. En efecto, si tomamos $f(x)=h(x)-\frac{c}{2}$ entonces

$$
h(x)+h(y)-c=f(x)+f(y)
$$

y

$$
f(x)=c-g(x)-\frac{c}{2}=\frac{c}{2}-g(x)<\frac{c}{2}<\frac{1}{2} .
$$

Ejemplo 2.6.2. Si en la Proposición 2.6.1 tomamos $X=] 0, \frac{1}{2}[, h(x)=1-x$ y $g(x)=x$, tenemos

$$
M(x, y)= \begin{cases}1, & \text { si } x=y \\ 1-x-y, & \text { si } x \neq y\end{cases}
$$

Entonces $(M, \mathfrak{L})$ es una métrica fuzzy estacionaria sobre $X$. (Caso particular de la Proposición 2.6.1) pero $(M, \cdot)$ no lo es. Para verificarlo, basta tomar $x=0,4, y=0,001$ y $z=0,45$, y asi tenemos que

$$
\begin{gathered}
M(x, z)=1-0,4-0,45<M(x, y) \cdot M(y, z)= \\
=(1-0,4-0,001) \cdot(1-0,001-0,45)=0,599 \cdot 0,549=0,3288 .
\end{gathered}
$$

Veamos que la topología de $(X, M, \mathfrak{L})$ es la topología discreta.

$$
\begin{gathered}
B(x, r)=\{y \in X: M(x, y)>1-r\} \cup\{x\}= \\
=\{y \in X: 1-x-y>1-r\} \cup\{x\}=\{y \in X: y<r-x\}= \\
=\{x\} \cup] 0, r-x[\text { si } r>x
\end{gathered}
$$

Si tomamos $r<x$, vemos claramente que $B(x, r)=\{x\}$ y por lo tanto la topología asociada a la métrica $M$ es la topología discreta. 
Proposición 2.6.3. Sean $f, g: X \rightarrow] 0, \frac{1}{2}[$ tales que $f>g y(f-g)(x)=c$ con $0<c<\frac{1}{2}, \forall x \in X$. Definimos $M$ por

$$
M(x, y)= \begin{cases}1, & \text { si } x=y \\ f(x)+g(y), & \text { si } x \neq y\end{cases}
$$

Entonces $(M, \mathfrak{L})$ es una métrica fuzzy estacionaria sobre $X$.

\section{Demostración.}

Las propiedades $(G V 1),(G V 2)$ y $(G V 5)$ son evidentes.

(GV3) Veamos que $M(x, y)=M(y, x)$. Para ello tenemos que ver que

$$
f(x)+g(y)=f(y)+g(x),
$$

que es lo mismo que probar que

$$
f(x)+g(y)-f(y)-g(x)=0 .
$$

En efecto,

$$
f(x)-g(x)-(f(y)-g(y))=c-c=0 .
$$

$(G V 4)$ Veamos que

$$
M(x, z) \geq \max \{0, M(x, y)+M(y, z)-1\} .
$$

Sean $x, y, z \in X$. Supondremos que $M(x, y)+M(y, z)>1$, de lo contrario la desigualdad es obvia.

Si $x \neq y \neq z \neq x$,

$$
M(x, y)+M(y, z)-1=f(x)+g(y)+f(y)+g(z)-1<
$$




$$
<f(x)+\frac{1}{2}+\frac{1}{2}+g(z)-1=f(x)+g(z)=M(x, z) .
$$

Si $x=z \neq y$, la desigualdad es obvia.

Si $x=y \neq z$, se da claramente la igualdad, pues

$M(x, y)+M(y, z)-1=1+f(y)+g(z)-1=f(x)+g(z)=M(x, z)$.

Si $x \neq y=z$, también se da la igualdad, ya que

$$
M(x, y)+M(y, z)-1=f(x)+f(y)+1-1=f(x)+f(z)=M(x, z) .
$$

Nota 2.6.4. Obsérvese que la ecuación (2.14) también se puede escribir como

$$
M(x, y)= \begin{cases}1, & \text { si } x=y \\ g(x)+g(y)+c, & \text { si } x \neq y\end{cases}
$$

\subsection{Métricas fuzzy definidas por t-normas conti- nuas}

Proposición 2.7.1. Sea $f: X \rightarrow] 0,1]$ una función tal que $f(x) * f(y) \neq 0$, $\forall x, y \in X$. Definimos la función $M$ por

$$
M(x, y)= \begin{cases}1, & \text { si } x=y \\ f(x) * f(y), & \text { si } x \neq y\end{cases}
$$

Entonces $(M, *)$ es una métrica fuzzy estacionaria sobre $X$. 


\section{Demostración.}

Las propiedades $(G V 1)-(G V 3)$ y $(G V 5)$ son evidentes.

Veamos (GV4). Para cada $x, y, z \in X$ tenemos que ver que

$$
M(x, y) * M(y, z) \leq M(x, z)
$$

En efecto

$$
M(x, y) * M(y, z)=f(x) * f(y) * f(y) * f(z) \leq f(x) * f(z)=M(x, z) .
$$

Ejemplo 2.7.2. En particular, si en la Proposición 2.7.1 tomamos $X \subset] 0,1]$ y $f: X \rightarrow] 0,1]$ es la función identidad, tenemos

$$
M(x, y)= \begin{cases}1, & \text { si } x=y \\ x \cdot y, & \text { si } x \neq y\end{cases}
$$

Entonces $(M, \cdot)$ es una métrica fuzzy estacionaria sobre $X$, pero $(M, \wedge)$ no lo es. Para verlo, basta tomar $x=0,1, y=0,9 y z=0,2$ y así

$$
\begin{gathered}
M(x, z)=x \cdot z<0,02<\min \{x y, y z\}= \\
=\min \{0,09 ; 0,18\}=0,09=\min \{M(x, y), M(y, z)\}
\end{gathered}
$$

Nota 2.7.3. Las bolas abiertas en $(X, M, \cdot)$ son de la forma

$$
\begin{gathered}
B(x, r, t)=\{y \in X: M(x, y)>1-r\}= \\
\left.\left.=\{y \in X: x \cdot y>1-r\}=\left\{y \in X: y>\frac{1-r}{x}\right\}=\right] \frac{1-r}{x}, 1\right] \cup\{x\}
\end{gathered}
$$

Tomando $r$ tal que $1-r>x$, se tiene que $\frac{1-r}{x}>1$ y entonces $B(x, r, t)=$ $\{x\}$, con lo cual $\tau_{M}$ es la topología discreta. 
Ejemplo 2.7.4. Si ahora tomamos $X=] 1,+\infty\left[\right.$ y $f(x)=\frac{1}{x}$, tenemos

$$
M(x, y)= \begin{cases}1, & \text { si } x=y \\ \frac{1}{x \cdot y}, & \text { si } x \neq y\end{cases}
$$

Entonces $(M, \cdot)$ es una métrica fuzzy estacionaria sobre $X$, (pues cumple claramente las condiciones de la Proposición 2.7.1) pero $(M, \wedge)$ no lo es. Para verlo, es suficiente tomar $x=10, y=2 y z=20, y$ asi tenemos que

$$
M(x, z)=\frac{1}{10} \cdot \frac{1}{20}=\frac{1}{200}<\min \left\{\frac{1}{20}, \frac{1}{40}\right\}=\frac{1}{40}=\min \{M(x, y), M(y, z)\}
$$

Nota 2.7.5. Dado $x \in X$,

$$
\begin{gathered}
B(x, r, t)=\{y \in X: M(x, y)>1-r\}= \\
=\left\{y \in X: \frac{1}{x y}>1-r\right\}=\left\{y \in X: y<\frac{1}{x(1-r)}\right\}
\end{gathered}
$$

Tomando $r \in] 0,1\left[\right.$ tal que $1-r>\frac{1}{x}$, tendremos $q u B(x, r, t)=\{x\}$, con lo que la topología asociada a la métrica $M$ es la topología discreta.

Ejemplo 2.7.6. Si tomamos $X=] 0, \pi[y f(x)=\operatorname{sen} x$ entonces tendremos

$$
M(x, y)= \begin{cases}1, & \text { si } x=y \\ \operatorname{sen} x \cdot \operatorname{sen} y, & \text { si } x \neq y\end{cases}
$$

Entonces $(M, \cdot)$ es una métrica fuzzy estacionaria sobre $X$ ya que $f(x)=$ $\operatorname{sen} x$ cumple que $f(x) \cdot f(y)=\operatorname{sen} x \cdot \operatorname{sen} y \neq 0, \forall x, y \in] 0, \pi[$, y $f$ toma valores en $] 0,1]$, pero $(M, \wedge)$ no lo es.

Para probar que $(M, \wedge)$ no es una métrica fuzzy, basta tomar $x=\frac{\pi}{4}$, $y=\frac{\pi}{2} y z=\frac{\pi}{6}, y$ asi

$M(x, z)=\operatorname{sen} \frac{\pi}{4} \cdot \operatorname{sen} \frac{\pi}{6}=\frac{\sqrt{2}}{2} \cdot \frac{1}{2}=\frac{\sqrt{2}}{4}<\min \left\{\operatorname{sen} \frac{\pi}{4} \cdot \operatorname{sen} \frac{\pi}{2}, \operatorname{sen} \frac{\pi}{6} \cdot \operatorname{sen} \frac{\pi}{2}\right\}=$ 


$$
=\min \left\{\frac{\sqrt{2}}{2}, \frac{1}{2}\right\}=\min \{M(x, y), M(y, z)\}
$$

Nota 2.7.7. Dado $x \in X=] 0, \pi[$,

$$
\begin{gathered}
B(x, r, t)=\{y \in X: M(x, y)>1-r\}= \\
=\{y \in X: \operatorname{sen} x \cdot \operatorname{sen} y>1-r\}=\left\{y \in X: \operatorname{sen} y>\frac{1-r}{\operatorname{sen} x}\right\}
\end{gathered}
$$

Tomando $r>1-\operatorname{sen} x$, entonces $\frac{1-r}{\operatorname{sen} x}>1$ y asi $B(x, r, t)=\{x\}$, con lo que $\tau_{M}$ es la topología discreta.

\subsection{Métricas fuzzy definidas por métricas fuzzy}

Proposición 2.8.1. Sean $(N, \cdot)$ una métrica fuzzy sobre $X$ y $\varphi:] 0,+\infty[\rightarrow$ $\mathbb{R}^{+}$una función continua y creciente. Definimos $M$ por

$$
M(x, y, t)=\frac{\varphi(t)+N(x, y, t)}{\varphi(t)+1}
$$

Entonces $(M, \cdot)$ es una métrica fuzzy sobre $X$.

\section{Demostración.}

Las propiedades $(G V 1)-(G V 3)$ y $(G V 5)$ son evidentes.

Veamos $(G V 4)$. Sean $a, b \in] 0,1]$. Como $\varphi$ es creciente, es fácil ver que

$$
\frac{\varphi(t)+a}{\varphi(t)+1} \cdot \frac{\varphi(s)+b}{\varphi(s)+1} \leq \frac{\varphi(t+s)+a}{\varphi(t+s)+1} \cdot \frac{\varphi(t+s)+b}{\varphi(t+s)+1} \leq \frac{\varphi(t+s)+a b}{\varphi(t+s)+1}
$$

Entonces, para $x, y, z \in X, t>0$, tenemos

$$
M(x, y, t) \cdot M(y, z, s)=\frac{\varphi(t)+N(x, y, t)}{\varphi(t)+1} \cdot \frac{\varphi(s)+N(y, z, s)}{\varphi(s)+1} \leq
$$




$$
\begin{gathered}
\leq \frac{\varphi(t+s)+N(x, y, t) \cdot N(y, z, s)}{\varphi(t+s)+1} \leq \\
\leq \frac{\varphi(t+s)+N(x, z, t+s)}{\varphi(t+s)+1}=M(x, z, t+s)
\end{gathered}
$$

Proposición 2.8.2. Sean $(N, *)$ una métrica fuzzy sobre $X$ y $\left.\left.\varphi: \mathbb{R}^{+} \rightarrow\right] 0,1\right]$ una función continua y creciente. Supongamos que $N(x, y, t) * \varphi(t) \neq 0$, $\forall x, y \in X, t>0$. Definimos la función $M$ por

$$
M(x, y, t)= \begin{cases}1, & \text { si } x=y \\ N(x, y, t) * \varphi(t), & \text { si } x \neq y\end{cases}
$$

Entonces $(M, *)$ es una métrica fuzzy sobre $X$.

\section{Demostración.}

Las propiedades $(G V 1)-(G V 3)$ y $(G V 5)$ son evidentes.

Veamos $(G V 4)$. Para ello tenemos que probar que

$$
M(x, y, t) * M(y, z, s) \leq M(x, z, t+s) .
$$

Sean $x, y, z \in X, t>0$. En los casos en que $x=z$, ó $x=y$ ó $y=z$, la desigualdad es obvia.

Supongamos que $x \neq y \neq z \neq x$. Entonces se tiene que

$$
\begin{gathered}
M(x, y, t) * M(y, z, s)=N(x, y, t) * \varphi(t) * N(y, z, s) * \varphi(s)= \\
=N(x, y, t) * N(y, z, s) * \varphi(t) * \varphi(s) \leq
\end{gathered}
$$




$$
\begin{gathered}
\leq N(x, z, t+s) * \varphi(t) * \varphi(s) \leq \\
\leq N(x, z, t+s) * \varphi(t+s)=M(x, z, t+s) .
\end{gathered}
$$

La última desigualdad es cierta porque $\varphi$ es creciente.

Así pues, tenemos probada la propiedad $(G V 4)$ y por lo tanto que $(M, *)$ es una métrica fuzzy sobre $X$.

Nota 2.8.3. Veamos ahora que $\tau_{M}$ es la topología discreta. Sea $t_{0}>0$ un valor real que verifica $\varphi\left(t_{0}\right)<1$. Se tiene que

$$
B_{M}\left(x, r, t_{0}\right)=\left\{y \in X: N\left(x, y, t_{0}\right) * \varphi\left(t_{0}\right)>1-r\right\} \cup\{x\} .
$$

Si elegimos ahora $r \in] 0,1\left[\right.$ de manera que $1-r>\varphi\left(t_{0}\right)$, entonces $B_{M}\left(x, r, t_{0}\right)=\{x\}$ y por tanto $\tau_{M}$ es la topología discreta.

Ejemplo 2.8.4. Como caso particular, si en la Proposición 2.8.2 tomamos $X=\{1\} \cup\left\{1-\frac{1}{n+1}: n \in \mathbb{N}\right\}$ y tomamos $\left.\left.\varphi: \mathbb{R}^{+} \rightarrow\right] 0,1\right]$ como

$$
\varphi(t)= \begin{cases}t, & \text { si } t<1 \\ 1, & \text { si } t \geq 1\end{cases}
$$

y $N$ es la métrica fuzzy sobre $X$ de la ecuación (2.16) definida por

$$
N(x, y)= \begin{cases}1, & \text { si } x=y \\ f(x) \cdot f(y), & \text { si } x \neq y\end{cases}
$$

Entonces tenemos

$$
M(x, y, t)= \begin{cases}1, & \text { si } x=y \\ t x y, & \text { si } x \neq y, t<1 \\ x y, & \text { si } x \neq y, t \geq 1\end{cases}
$$


Así, $(M, \cdot)$ es una métrica fuzzy sobre $X$ (Ejemplo 19 de [24]). Además, para cada $x \in X, B\left(x, \frac{1}{2}, \frac{1}{2}\right)=\{x\}$, por lo tanto $\tau_{M}$ es la topología discreta en $X$.

Proposición 2.8.5. Sea $(X, N, \cdot)$ un espacio métrico fuzzy estacionario y $h: \mathbb{R}^{+} \rightarrow \mathbb{R}^{+}$una función continua y creciente. Definimos la función $M$ por

$$
M(x, y, t)=\frac{h(t)}{h(t)+1-N(x, y, t)}
$$

Entonces $(M, \cdot)$ es una métrica fuzzy sobre $X$.

\section{Demostración.}

Las propiedades $(G V 1)-(G V 3)$ y $(G V 5)$ son evidentes.

Veamos (GV4). Sean $x, y, z \in X$. Veamos que

$$
M(x, z, t+s) \geq M(x, y, t) \cdot M(y, z, s) .
$$

Para cada $x, y, z \in X$ y $t>0$, tenemos

$$
(1-N(x, y))(1-N(y, z))=1+N(x, y) N(y, z)-N(x, y)-N(y, z) \geq 0
$$

y así

$\varphi(t)(1-N(x, y))(1-N(y, z))+1+N(x, y) N(y, z)-N(x, y)-N(y, z) \geq 0$.

Ahora tenemos:

$$
(\varphi(t)+1-N(x, y))(\varphi(t)+1-N(y, z)) \geq \varphi(t)(\varphi(t)+1-N(x, z))
$$

o equivalentemente

$$
\frac{1}{\varphi(t)+1-N(x, z)} \geq \frac{1}{\varphi(t)+1-N(x, y)} \cdot \frac{\varphi(t)}{\varphi(t)+1-N(y, z)}
$$


y por lo tanto

$$
\frac{\varphi(t)}{\varphi(t)+1-N(x, z)} \geq \frac{\varphi(t)}{\varphi(t)+1-N(x, y)} \cdot \frac{\varphi(t)}{\varphi(t)+1-N(y, z)}
$$

y como consecuencia

$$
\begin{gathered}
\frac{\varphi(t+s)}{\varphi(t+s)+1-N(x, z)} \geq \frac{\varphi(t+s)}{\varphi(t+s)+1-N(x, y)} \cdot \frac{\varphi(t+s)}{\varphi(t+s)+1-N(y, z)} \geq \\
\geq \frac{\varphi(t)}{\varphi(t)+1-N(x, y)} \cdot \frac{\varphi(s)}{\varphi(s)+1-N(y, z)}
\end{gathered}
$$

ya que $\varphi$ es creciente, y por lo tanto tenemos probado que

$$
M(x, z, t+s) \geq M(x, y, t) \cdot M(y, z, s) .
$$

\subsection{Métricas fuzzy definidas por particiones}

Proposición 2.9.1. Sea $\left.\left.\varphi: \mathbb{R}^{+} \rightarrow\right] 0,1\right]$ una función continua y creciente y sea $X=A \cup B$, con $A \cap B=\phi$. Sea $(N, *)$ una métrica fuzzy sobre $X$ tal que $N(x, y, t) * \varphi(t) \neq 0, \forall x, y \in X$ y $t>0$. Definimos la función $M$ por

$$
M(x, y, t)= \begin{cases}N(x, y, t), & \text { si } x, y \in A \text { ó } x, y \in B \\ N(x, y, t) * \varphi(t), & \text { en otro caso }\end{cases}
$$

Entonces $(M, *)$ es una métrica fuzzy sobre $X$.

Demostración. 
Las propiedades $(G V 1)-(G V 3)$ y $(G V 5)$ son evidentes.

$(G V 4)$ Veamos que $M(x, y, t) * M(y, z, s) \leq M(x, z, t+s)$

Si $x, y, z \in A$ ó $x, y, z \in B$, la prueba es obvia pues $N$ es una métrica fuzzy.

Si $x, y \in A$ y $z \in B$ ó $x, y \in B$ y $z \in A$, entonces

$$
\begin{gathered}
M(x, y, t) * M(y, z, s)=N(x, y, t) * N(y, z, s) * \varphi(t) \leq N(x, z, t+s) * \varphi(t) \leq \\
\leq N(x, z, t+s) * \varphi(t+s)=M(x, z, t+s)
\end{gathered}
$$

Si $x, z \in A$ e $y \in B$ ó $x, z \in B$ e $y \in A$, entonces

$$
\begin{gathered}
M(x, y, t) * M(y, z, s)=N(x, y, t) * \varphi(t) * N(y, z, s) * \varphi(s)= \\
=N(x, y, t) * N(y, z, s) * \varphi(t) * \varphi(s) \leq N(x, y, t) * N(y, z, s) * 1 * 1= \\
=N(x, y, t) * N(y, z, s) * 1=N(x, z, t+s)=M(x, z, t+s)
\end{gathered}
$$

Nota 2.9.2. Vamos a determinar ahora $B(x, r, t)$. Supongamos que $x \in A$, entonces

$$
\begin{gathered}
B(x, r, t)=\{y \in X: M(x, y, t)>1-r\}= \\
=\{y \in A: M(x, y, t)>1-r\} \cup\{y \in B: M(x, y, t)>1-r\}= \\
=\{y \in A: N(x, y, t)>1-r\} \cup\{y \in B: N(x, y, t) * \varphi(t)>1-r\}
\end{gathered}
$$

Si tomamos $t_{0}>0$ tal que $\varphi\left(t_{0}\right)<1$ y $r$ tal que $1-r>\varphi\left(t_{0}\right)$, entonces $\tau_{N_{A}} \cup\{x\}$, es decir, la topología es la misma que $\tau_{N}$ pero restringida a la partición $A$. 
Ejemplo 2.9.3. Como caso particular de la Proposición 2.9.1, consideremos la siguiente métrica fuzzy $M$. Sea $N$ el conjunto fuzzy sobre $] 0,1]^{2} \times \mathbb{R}^{+}$ definido por

$$
N(x, y)= \begin{cases}1, & \text { si } x=y \\ x * y, & \text { si } x \neq y\end{cases}
$$

donde $x * y \neq 0, \forall x, y \in] 0,1]$. Según la Proposición 2.7.1, $(N, \cdot)$ es una métrica fuzzy sobre ]0,1]. Sean $A, B \subset] 0,1]$ tales que $A \cup B=] 0,1]$ y $A \cap B=\phi$ y consideremos la función $\left.\left.\varphi: \mathbb{R}^{+} \rightarrow\right] 0,1\right]$ definida por

$$
\varphi(t)= \begin{cases}t, & \text { si } 0<t<1 \\ 1, & \text { si } t \geq 1\end{cases}
$$

Definimos la función $M$ por

$$
M(x, y, t)= \begin{cases}1, & \text { si } x=y \\ x * y, & \text { si } x, y \in A \text { ó } x, y, \in B \\ x * y * \varphi(t) & \text { en otro caso }\end{cases}
$$

Entonces $(M, *)$ es una métrica fuzzy sobre $X$.

\subsection{Extensión de una métrica fuzzy}

\subsubsection{Extensión de dos métricas fuzzy coincidentes en su codominio}

Proposición 2.10.1. Sean $H$ y $K$ dos conjuntos distintos con $H \cap K \neq \phi$. Si $\left(M_{H}, *\right)$ y $\left(M_{K}, *\right)$ son métricas fuzzy estacionarias sobre $H$ y $K$ respectivamente, que coinciden en $H \cap K$, entonces existe una métrica fuzzy estacionaria $M$ sobre $H \cup K$ tal que $M$ restringido a $H$ es $M_{H}$ y $M$ restringido a $K$ es $M_{K}$. 


\section{Demostración.}

Sean $x, y \in H \cup K$ y $t>0$.

Si $x, y \in H$, definimos $M(x, y)=M_{H}(x, y)$.

Si $x, y \in K$, definimos $M(x, y)=M_{K}(x, y)$.

Si $x \in H \backslash K$, e $y \in K \backslash H$, definimos

$$
M(x, y)=\sup \left\{M_{H}(x, a) * M_{K}(a, y): a \in H \cap K\right\}
$$

Veamos que $M$ es una métrica fuzzy sobre $H \cup K$. Probaremos sólo la desigualdad triangular, ya que las otras propiedades son evidentes.

Sean $x, y, z \in H \cup K$

Si $x, y, z, \in H$ ó $x, y, z \in K$, la prueba es obvia. Veamos los casos no triviales.

(a) Supongamos que $x, y \in H$ y $z \in K \backslash H$. Entonces dado $\varepsilon>0$, podemos encontrar $a \in H \cap K$ tal que $M(y, z)-\varepsilon<M_{H}(y, a) * M_{K}(a, z)$. Por tanto

$$
\begin{gathered}
M(x, y) *(M(y, z)-\varepsilon) \leq M(x, y) * M_{H}(y, a) * M(a, z)= \\
=M_{H}(x, y) * M_{H}(y, a) * M_{K}(a, z) \leq M_{H}(x, a) * M_{K}(a, z) \leq M(x, z)
\end{gathered}
$$

Como $\varepsilon$ es arbitrario $\mathrm{y} *$ es continua, tenemos que

$$
M(x, y) * M(y, z) \leq M(x, z) .
$$

El caso $x, y \in K$ y $z \in H \backslash K$ se prueba de manera similar. 
(b) Supongamos que $x, z \in H$ e $y \in K \backslash H$. Entonces dado $\varepsilon>0$, podemos encontrar $a, b \in H \cap K$ tales que

$$
M(x, y)-\varepsilon<M_{H}(x, a) * M_{K}(a, y)
$$

y

$$
M(y, z)-\varepsilon<M_{K}(y, b) * M_{H}(b, z)
$$

Por lo tanto

$$
\begin{gathered}
(M(x, y)-\varepsilon) *(M(y, z)-\varepsilon) \leq M_{H}(x, a) * M_{K}(a, y) * M_{K}(y, b) * M_{H}(b, z) \leq \\
\leq M_{H}(x, a) * M_{K}(a, b) * M_{H}(b, z)=M_{H}(x, a) * M_{H}(a, b) * M_{H}(b, z) \leq \\
\leq M_{H}(x, z)=M(x, z) .
\end{gathered}
$$

Como $\varepsilon$ es arbitrario $\mathrm{y} *$ es continua, tenemos que

$$
M(x, y) * M(y, z) \leq M(x, z) .
$$

El caso $x, z \in K, y \in H \backslash K$ es similar.

Ejemplo 2.10.2. Sean $H=\{1,3,7\}$ y consideremos la función $M_{H}$ sobre $H \times H$ dada por

$$
M_{H}(x, y)=\frac{m c d(x, y)}{m c m(x, y)}
$$

Entonces

$$
\begin{gathered}
M_{H}(1,1)=M_{H}(3,3)=M_{H}(7,7)=1 \\
M_{H}(1,3)=M_{H}(3,1)=\frac{1}{3} \\
M_{H}(1,7)=M_{H}(7,1)=\frac{1}{7} \\
M_{H}(3,7)=M_{H}(7,3)=\frac{1}{21}
\end{gathered}
$$

Está claro que $\left(H, M_{H}, \cdot\right)$ es un espacio métrico fuzzy estacionario. 
Sea $K=\{1,4,9\}$ y consideremos la función $M_{K}$ sobre $K \times K$ dada por la ecuación (2.21). Entonces

$$
\begin{gathered}
M_{K}(1,1)=M_{K}(4,4)=M_{K}(9,9)=1 \\
M_{K}(1,4)=M_{K}(4,1)=\frac{1}{4} \\
M_{K}(1,9)=M_{K}(9,1)=\frac{1}{9} \\
M_{K}(4,9)=M_{K}(9,4)=\frac{1}{36}
\end{gathered}
$$

Está claro que $\left(K, M_{K}, \cdot\right)$ es un espacio métrico fuzzy estacionario y además si tomamos $r \in] 0,1\left[\right.$ tal que $1-r<\frac{1}{4}$ obtenemos que $B(x, r, t)=\{x\}$, con lo cual la topología asociada a $M_{K}$ es la topología discreta. Además tenemos que $H \cap K=\{1\}$ y obviamente $M_{H}$ y $M_{K}$ coinciden en $H \cap K$. Entonces, por la Proposición 2.10.1, podemos definir la métrica fuzzy estacionaria $M$ sobre $H \cup K$ de la siguiente forma:

$$
\begin{aligned}
& M(3,4)=M(4,3)=M_{H}(3,1) \cdot M_{K}(1,4)=\frac{1}{3} \cdot \frac{1}{4}=\frac{1}{12} \\
& M(3,9)=M(9,3)=M_{H}(3,1) \cdot M_{K}(1,9)=\frac{1}{3} \cdot \frac{1}{9}=\frac{1}{27} \\
& M(7,4)=M(4,7)=M_{H}(7,1) \cdot M_{K}(1,4)=\frac{1}{7} \cdot \frac{1}{4}=\frac{1}{28} \\
& M(7,9)=M(9,7)=M_{H}(7,1) \cdot M_{K}(1,9)=\frac{1}{7} \cdot \frac{1}{9}=\frac{1}{63}
\end{aligned}
$$

$y$

$$
M(x, y)=M_{H}(x, y) \text { si } x, y \in H \text { y } M(x, y)=M_{K}(x, y) \text { si } x, y \in K \text {. }
$$

Ejemplo 2.10.3. Sea $\left(M_{H}, \cdot\right)$ la métrica fuzzy estacionaria sobre $H=$ $\{1,3,7\}$ del Ejemplo 2.10.2. Sea $K=\mathbb{N} \backslash\{7\}$ y consideremos sobre $K$ la métrica fuzzy estacionaria dada por

$$
M_{K}(n, m)=\frac{\min \{n, m\}}{\max \{n, m\}}
$$


Asi tenemos que $H \cap K=\{1,3\}$ y obviamente $M_{H}$ y $M_{K}$ coinciden en $H \cap K$. Entonces, por la Proposición 2.10.1 podemos definir la métrica fuzzy estacionaria $M$ sobre $\mathbb{N}$ de la siguiente forma:

Si $n \neq 7, n>3$, definimos

$$
\begin{gathered}
M(n, 7)=\max \left\{\frac{\min \{n, 1\}}{\max \{n, 1\}} \cdot M_{H}(1,7), \frac{\min \{n, 3\}}{\max \{n, 3\}} \cdot M_{H}(3,7)\right\}= \\
=\max \left\{\frac{1}{n} \cdot \frac{1}{7}, \frac{3}{n} \cdot \frac{1}{21}\right\}=\frac{1}{7 n} \\
M(2,7)=\max \left\{\frac{\min \{2,1\}}{\max \{2,1\}} \cdot M_{H}(1,7), \frac{\min \{2,3\}}{\max \{2,3\}} \cdot M_{H}(3,7)\right\}= \\
=\max \left\{\frac{1}{2} \cdot \frac{1}{7}, \frac{2}{3} \cdot \frac{1}{21}\right\}=\frac{1}{14}
\end{gathered}
$$

$$
\begin{aligned}
& \text { Por lo tanto } M(n, 7)=M(7, n)=\frac{1}{7 n}, \forall n \neq 7, y \\
& M(n, m)=\frac{\min \{n, m\}}{\max \{n, m\}}, \text { en cualquier otro caso. }
\end{aligned}
$$

Esta métrica así definida es claramente una métrica fuzzy sobre $\mathbb{N}=H \cup K$ por la Proposición 2.10.1.

Observar que el elemento más "cercano", respecto $M$, a $n \in X$ es siempre $n+1$.

La topología $\tau_{M}$ es la discreta. En efecto, para cada $n \in X$, si tomamos $0<r<1-\frac{n}{n+1}$, se tiene que $B(n, r, t)=\{n\}$. 


\subsubsection{Métricas fuzzy definidas por isometrías}

Sean $M$ y $N$ dos métricas fuzzy sobre $X$ e $Y$ respectivamente. Recordemos que la biyección $f:(X, M) \rightarrow(Y, N)$ se llama isometría si para cada $x, y \in X$ y $t>0$

$$
N(f(x), f(y), t)=M(x, y, t)
$$

Si $f$ es una isometría, obviamente $f^{-1}$ es una isometría de $Y$ en $X$.

Omitimos la prueba de la siguiente proposición.

Proposición 2.10.4. Supongamos que $(M, *)$ es una métrica fuzzy sobre $X$ y $f$ una biyección de $X$ en $Y$. Entonces podemos definir en $Y$ una métrica fuzzy $(N, *)$ mediante la ecuación (2.22).

Podemos decir que la métrica fuzzy $M$ ha sido transportada de $X$ a $Y$, es decir, $(Y, N, *)$ es un espacio métrico fuzzy.

Como caso particular, consideremos en $\mathbb{R}^{+}$la métrica fuzzy estacionaria del Ejemplo 2.10.3

$$
M(x, y)=\frac{\min \{x, y\}}{\max \{x, y\}}
$$

y sea $f: \mathbb{R} \rightarrow \mathbb{R}^{+}$la función exponencial, es decir, $f(x)=e^{x}, \forall x \in \mathbb{R}$. Definimos

$$
N(x, y)=\frac{\min \left\{e^{x}, e^{y}\right\}}{\max \left\{e^{x}, e^{y}\right\}}
$$

Entonces $(N, \cdot)$ es una métrica fuzzy estacionaria sobre $\mathbb{R}$, y la topología $\tau_{N}$ coincide con la topología usual de $\mathbb{R}$. 


\section{Capítulo 3}

\section{Métricas fuzzy principales}

\subsection{Introducción}

El concepto de p-convergencia en espacios métricos fuzzy, en el sentido de George y Veeramani, ha sido dado por D. Mihet en [38]. En la Sección 3.2 de este capítulo estudiamos algunos aspectos relativos a este concepto y caracterizaremos aquellos espacios métricos fuzzy, que llamamos principales, en los que la familia de sucesiones p-convergentes coincide con la familia de sucesiones convergentes. También se da un espacio métrico fuzzy no completable, que no es principal. En la primera parte de la Sección 3.2 empezamos presentando (Definición 3.2.1) el concepto de p-convergencia que el autor dió en [38] y seguidamente vemos las primeras propiedades relativas a dicha definición. A continuación damos en la Definición 3.2.7 el concepto de espacio métrico fuzzy principal (un espacio métrico fuzzy $(X, M, *)$ es principal si $\{B(x, r, t): r \in] 0,1[\}$ es una base local de $x \in X$, para cada $x \in X$ y $t>0$, es decir, un sistema fundamental de entornos) y una serie de ejemplos que 
nos ilustran dicho concepto (Ejemplo 3.2.8). El resultado más relevante es el Teorema 3.2.9, el cual dice que un espacio métrico fuzzy $(X, M, *)$ principal se caracteriza porque toda sucesión p-convergente es convergente. Finalmente, en el Ejemplo 3.2.11 vemos un espacio métrico fuzzy completo que no es principal.

En la segunda parte de la Sección 3.2, continuamos con el estudio iniciado por D. Mihet y vemos la definición de sucesión de p-Cauchy (Definición 3.2.13. Una sucesión $\left\{x_{n}\right\}_{n \in \mathbb{N}}$ en el espacio métrico fuzzy $(X, M, *)$ se dice que es p-Cauchy si para cada $\varepsilon \in] 0,1\left[\right.$ existen $n_{0} \in \mathbb{N}$ y $t_{0}>0$ tales que $M\left(x_{n}, x_{m}, t_{0}\right)>1-\varepsilon$ para todo $n, m \geq n_{0}$, es decir, $\lim _{n, m \rightarrow \infty} M\left(x_{n}, x_{m}, t_{0}\right)=1$ para algún $t_{0}>0$ ), después damos el concepto de p-completo, en la Definición 3.2.14. (Un espacio métrico fuzzy $(X, M, *)$ se llama p-completo si toda sucesión p-Cauchy en $X$ es p-convergente a algún punto de $X$ ). Obviamente los conceptos de p-completitud y completitud son conceptos equivalentes en métricas fuzzy estacionarias y en la Proposición 3.2.15 vemos que la métrica fuzzy estándar $M_{d}$ es p-completa si y sólo si $M_{d}$ es completa.

En la Sección 3.3 estudiamos un nuevo concepto relacionado a las aplicaciones continuas entre espacios métricos fuzzy en el sentido de George y Veeramani, que son las aplicaciones t-continuas, y vemos algunas propiedades sobre ellas, en especial cuando el espacio métrico fuzzy de partida es principal. Una aplicación entre espacios métricos fuzzy $f:(X, M) \longrightarrow(Y, N)$ es t-continua en $x_{0} \in X$ si dados $\left.\varepsilon \in\right] 0,1[$ y $t>0$ existe $\delta \in] 0,1[$ tal que si $M\left(x_{0}, x, t\right)>1-\delta$, entonces $N\left(f\left(x_{0}\right), f(x), t\right)>1-\varepsilon$. (Definición 3.3.2). Obviamente, si $f$ es t-continua en $x_{0}$ entonces $f$ es continua en $x_{0}$, pero en el Ejemplo 3.3.4 vemos que el recíproco es falso. La Proposición 3.3.6 muestra que las funciones continuas definidas sobre un espacio métrico fuzzy principal son t-continuas, es decir, la continuidad equivale a la t-continuidad. Como caso particular, esto sucede cuando el espacio inicial está provisto de 
la métrica fuzzy estándar, pues éstas son principales.

El contenido de este capítulo ha sido publicado en [26] y [27].

\subsection{Convergencia en espacios métricos fuzzy}

Definición 3.2.1. [38] Sea $(X, M, *)$ un espacio métrico fuzzy. Una sucesión $\left\{x_{n}\right\}_{n \in \mathbb{N}}$ en $X$ se llama punto convergente a $x_{0} \in X$ si $\lim _{n \rightarrow \infty} M\left(x_{n}, x_{0}, t_{0}\right)=1$ para algún $t_{0}>0$.

En este caso diremos que $\left\{x_{n}\right\}_{n \in \mathbb{N}}$ es p-convergente a $x_{0}$ para $t_{0}>0$,o simplemente, que $\left\{x_{n}\right\}_{n \in \mathbb{N}}$ es p-convergente.

Equivalentemente, $\left\{x_{n}\right\}_{n \in \mathbb{N}}$ es p-convergente si existe $x_{0} \in X$ y $t_{0}>0$ tal que $\left\{x_{n}\right\}_{n \in \mathbb{N}}$ está eventualmente en $B\left(x_{0}, r, t_{0}\right)$ para cada $\left.r \in\right] 0,1[$, (o sin pérdida de generalidad, en $B\left(x_{0}, \frac{1}{n}, t_{0}\right)$ para cada $\left.n \in \mathbb{N}\right)$.

Obviamente, $\left\{x_{n}\right\}_{n \in \mathbb{N}}$ es convergente a $x_{0}$ si y sólo si $\left\{x_{n}\right\}_{n \in \mathbb{N}}$ es pconvergente a $x_{0}$ para todo $t>0$. Las dos siguientes propiedades se dan a conocer en [38].

Proposición 3.2.2. [38] Sea $\left\{x_{n}\right\}_{n \in \mathbb{N}}$ una sucesión en un espacio métrico fuzzy $(X, M, *)$. Entonces:

(1) Si $\lim _{n \rightarrow \infty} M\left(x_{n}, x, t_{1}\right)=1 y \lim _{n \rightarrow \infty} M\left(x_{n}, y, t_{2}\right)=1$, entonces $x=y$.

(2) Si $\lim _{n \rightarrow \infty} M\left(x_{n}, x_{0}, t_{0}\right)=1$ entonces $\lim _{k \rightarrow \infty} M\left(x_{n_{k}}, x_{0}, t_{0}\right)=1$ para cada subsucesión $\left\{x_{n_{k}}\right\}_{k \in \mathbb{N}}$ de $\left\{x_{n}\right\}_{n \in \mathbb{N}}$.

Por la propiedad (1) obtenemos el siguiente corolario. 
Corolario 3.2.3. Si $\left\{x_{n}\right\}_{n \in \mathbb{N}}$ es p-convergente a $x_{0}$ y $\left\{x_{n}\right\}_{n \in \mathbb{N}}$ es convergente, entonces $\left\{x_{n}\right\}_{n \in \mathbb{N}}$ converge a $x_{0}$.

Demostración.

Como $\left\{x_{n}\right\}_{n \in \mathbb{N}}$ es p-convergente a $x_{0}$, entonces

$$
\lim _{n \rightarrow \infty} M\left(x_{n}, x_{0}, t_{0}\right)=1, \text { para algún } t_{0}>0 .
$$

Además $\left\{x_{n}\right\}_{n \in \mathbb{N}}$ converge, luego existe $x \in X$ tal que $\lim _{n \rightarrow \infty} M\left(x_{n}, x, t\right)=1$ para cada $t>0$. Luego por (1) de la Proposición 3.2.2 se tiene que $x_{0}=x \mathrm{y}$ así

$$
\lim _{n \rightarrow \infty} M\left(x_{n}, x_{0}, t\right)=1 \text { para cada } t>0,
$$

y por lo tanto $\left\{x_{n}\right\}_{n \in \mathbb{N}}$ converge a $x_{0}$.

Corolario 3.2.4. Sea $(X, M, *)$ un espacio métrico fuzzy completable. Si $\left\{x_{n}\right\}_{n \in \mathbb{N}}$ es una sucesión de Cauchy en $X$ y $\left\{x_{n}\right\}_{n \in \mathbb{N}}$ es p-convergente a $x_{0} \in X$, entonces $\left\{x_{n}\right\}_{n \in \mathbb{N}}$ converge a $x_{0}$.

\section{Demostración.}

Sea $\left(X^{*}, M^{*}\right)$ la completación de $(X, M)$. Entonces existe $x^{*} \in X^{*}$ tal que $\left\{x_{n}\right\}_{n \in \mathbb{N}}$ converge a $x^{*}$, es decir $\lim _{n \rightarrow \infty} M^{*}\left(x_{n}, x^{*}, t\right)=1, \forall t>0$.

Supongamos ahora que $\lim _{n \rightarrow \infty} M\left(x_{n}, x_{0}, t_{0}\right)=1$ para algún $t_{0}>0$. Entonces $\lim _{n \rightarrow \infty} M^{*}\left(x_{n}, x_{0}, t_{0}\right)=\lim _{n \rightarrow \infty} M\left(x_{n}, x_{0}, t_{0}\right)=1$, con lo cual $\left\{x_{n}\right\}_{n \in \mathbb{N}}$ es $M^{*}$-p-convergente a $x_{0}$, y por el corolario anterior $x^{*}=x_{0}$, es decir, $\left\{x_{n}\right\}_{n \in \mathbb{N}}$ converge a $x_{0}$.

Veamos un ejemplo de una sucesión p-convergente que no es convergente. 
Ejemplo 3.2.5. [38] Sea $\left.\left\{x_{n}\right\} \subset\right] 0,1[$ una sucesión estrictamente creciente y convergente a 1 respecto de la topología usual de $\mathbb{R}$ y sea $X=\left\{x_{n}\right\} \cup\{1\}$. Definimos la función $M$ sobre $X^{2} \times \mathbb{R}^{+}$dada por

$$
\begin{aligned}
& M(x, x, t)=1 \text { para cada } x \in X, t>0, \\
& M\left(x_{n}, x_{m}, t\right)=\min \left\{x_{n}, x_{m}\right\}, \text { para todo } m, n \in \mathbb{N}, t>0 y \\
& M\left(x_{n}, 1, t\right)=M\left(1, x_{n}, t\right)=\min \left\{x_{n}, t\right\}, \text { para todo } n \in \mathbb{N}, t>0 .
\end{aligned}
$$

Entonces $(M, *)$ es una métrica fuzzy sobre $X$, donde $a * b=\min \{a, b\}$. La sucesión $\left\{x_{n}\right\}_{n \in \mathbb{N}}$ no es convergente ya que $\lim _{n \rightarrow \infty} M\left(x_{n}, 1, \frac{1}{2}\right)=\frac{1}{2}$. Sin embargo es p-convergente a 1 ya que $\lim _{n \rightarrow \infty} M\left(x_{n}, 1,1\right)=1$

Nota 3.2.6. En el ejemplo anterior, $\{1\}$ es abierto en $\tau_{M}$ ya que $B\left(1, \frac{1}{2}, \frac{1}{2}\right)=$ $\{1\}$. Por otro lado, para $r \in] 0,1\left[\right.$ tenemos que $B(1, r, 1)=\left\{x_{m}, x_{m+1}, \ldots\right\} \cup$ $\{1\}$ donde $x_{m}$ es el primer elemento de $\left\{x_{n}\right\}_{n \in \mathbb{N}}$ tal que $0<1-r<x_{m}$. Por lo tanto, la familia de bolas abiertas $\{B(1, r, 1): r \in] 0,1[\}$ no es una base local de 1. Esto motiva nuestra siguiente definición.

Definición 3.2.7. Un espacio métrico fuzzy $(X, M, *)$ diremos que es principal (o simplemente, $M$ es principal) si para cualquier $t>0$, la familia $\{B(x, r, t): r \in] 0,1[\}$ es una base local de $x \in X$, para cada $x \in X$, es decir, un sistema fundamental de entornos de $x$.

Como acabamos de ver, la métrica fuzzy del Ejemplo 3.2.5 no es principal. A continuación vamos a ver algunos ejemplos de métricas fuzzy principales.

\section{Ejemplo 3.2.8.}

(a) Toda métrica fuzzy estacionaria es, obviamente, principal.

(b) La métrica fuzzy estándar es principal. 
(c) La métrica fuzzy

$$
M(x, y, t)=e^{-\frac{d(x, y)}{t}},
$$

donde d es una métrica sobre $X$, [14], es principal.

(d) La métrica fuzzy

$$
M(x, y, t)=\frac{\min \{x, y\}+t}{\max \{x, y\}+t},
$$

es una métrica fuzzy sobre $\mathbb{R}^{+}$, [55], la cual es principal.

\section{Demostración.}

(a) Es obvio.

(b) Sea $(X, d)$ un espacio métrico. Entonces se tiene que $B_{M_{d}}\left(x_{0}, \delta, t\right)=$ $B_{r}\left(x_{0}\right)$ si y sólo si $\frac{1-\delta}{t \cdot \delta}=\frac{1}{r}$.

En efecto, sean $\left.x_{0} \in X, t>0, \delta \in\right] 0,1[$ y $r>0$ que verifican lo anterior. Sea $x \in B_{M_{d}}\left(x_{0}, \delta, t\right)$. Entonces $M_{d}\left(x_{o}, x, t\right)>1-\delta$, es decir, $\frac{t}{t+d\left(x_{0}, x\right)}>1-\delta$ y por tanto $d\left(x_{0}, x\right)<t \cdot \frac{\delta}{1-\delta}=r$, es decir, $x \in B_{r}\left(x_{o}\right)$.

Análogamente se prueba la inclusión contraria.

Ahora observar que la relación $\frac{1-\delta}{t \cdot \delta}=\frac{1}{r}$ también se puede escribir $\delta=\frac{r}{t+r}$.

Finalmente veamos que la familia $\left\{B_{M_{d}}\left(x_{0}, \delta, t\right): \delta \in\right] 0,1[\}$ es un sistema fundamental de entornos de $x_{o} \in X$, para cualquier $t>0$.

En efecto, si $B_{r}\left(x_{o}\right)$ es una bola centrada en $x_{0}$, y si $t>0$, entonces eligiendo $\delta=\frac{r}{t+r}$, tenemos que $B_{d}\left(x_{0}, \delta, t\right)=B_{r}\left(x_{0}\right)$ según la afirmación anterior, y así $M_{d}$ es principal. 
(c) Para ver que $M$ es principal, basta elegir $\delta=1-\frac{1}{e^{\frac{\pi}{t}}}$ y se tiene que $B\left(x_{0}, \delta, t\right)=B_{r}\left(x_{0}\right)$.

Con un razonamiento similar al caso (b) se prueba que $M$ es principal.

(d) Es fácil verificar que dados $t>0$ y $\delta \in] 0,1\left[\right.$, las bolas abiertas en $\tau_{M}$ son de la forma $B(x, \delta, t)=](x+t) \cdot(1-\delta)-t, \frac{x+t}{1-\delta}-t[$.

Para cualquier $t>0$ se tiene que el diámetro de los intervalos anteriores tienden a cero cuando $\delta$ tiende a cero, pero $B(x, \delta, t)$ jamás de reduce al punto $\{x\}$.

Así pues, $\tau_{M}$ es la topología usual de $\mathbb{R}$ restringida a $X$, y por el razonamiento anterior $\{B(x, r, t): r \in] 0,1[\}$ es un sistema fundamental de entornos de $x \in X$, para cualquier $t>0$, y por tanto $M$ es principal.

Teorema 3.2.9. Un espacio métrico fuzzy $(X, M, *)$ es principal si y sólo si toda sucesión p-convergente es convergente.

\section{Demostración.}

Supongamos que $M$ es principal y que $\left\{x_{n}\right\}_{n \in \mathbb{N}}$ es p-convergente a $x_{0}$, para $t_{0}>0$.

Sea $\varepsilon \in] 0,1\left[\mathrm{y} t>0\right.$. Como $M$ es principal, entonces $\left\{B\left(x_{0}, \frac{1}{n}, t_{0}\right): n \in \mathbb{N}\right\}$ es una base local de $x_{0}$. Por lo tanto, podemos encontrar $m \in \mathbb{N}$ tal que $B\left(x_{0}, \frac{1}{m}, t_{0}\right) \subset B\left(x_{0}, \varepsilon, t\right)$.

Como $\lim _{n \rightarrow \infty} M\left(x_{n}, x_{0}, t_{0}\right)=1$, podemos encontrar $\left.\delta \in\right] 0,1\left[\right.$, con $\delta<\frac{1}{m}$, y $n_{1} \in \mathbb{N}$ tal que $x_{n} \in B\left(x_{0}, \delta, t_{0}\right)$ para todo $n \geq n_{1}$, y así $x_{n} \in B\left(x_{0}, \varepsilon, t\right)$ para todo $n \geq n_{1}$. Por lo tanto, $M\left(x_{n}, x_{0}, t\right)>1-\varepsilon$ para todo $n \geq n_{1}$, y así 
$\lim _{n \rightarrow \infty} M\left(x_{n}, x_{0}, t\right)=1$. El razonamiento anterior es válido para todo $t>0$, con lo cual $\left\{x_{n}\right\}_{n \in \mathbb{N}}$ converge a $x_{0}$.

Para el recíproco, supongamos que $M$ no es principal. Vamos a construir una sucesión p-convergente que no sea convergente.

Si $M$ no es principal, podemos encontrar $x_{0} \in X$ y $t_{0}>0$ tal que $\left\{B\left(x_{0}, \frac{1}{n}, t_{0}\right): n \in \mathbb{N}\right\}$ no es una base local de $x_{0}$. Entonces podemos encontrar $t>0$ y $r \in] 0,1\left[\right.$ tal que $B\left(x_{0}, \frac{1}{n}, t_{0}\right) \nsubseteq B\left(x_{0}, r, t\right)$ para todo $n \in \mathbb{N}$.

Ahora, por inducción, construimos la siguiente sucesión.

Para cada $n \in \mathbb{N}$, tomamos $x_{n} \in B\left(x_{0}, \frac{1}{n}, t_{0}\right) \backslash B\left(x_{0}, r, t\right)$.

Ahora, dado $\varepsilon \in] 0,1$, elegimos $n_{1} \in \mathbb{N}$ con $\frac{1}{n_{1}}<\varepsilon$. Por lo tanto, para $m \geq n_{1}$, tenemos que

$$
M\left(x_{m}, x_{0}, t_{0}\right)>1-\frac{1}{m}>1-\frac{1}{n_{1}}>1-\varepsilon
$$

y como $\varepsilon$ es arbitrario, $\lim _{n \rightarrow \infty} M\left(x_{n}, x_{0}, t_{0}\right)=1$ y así $\left\{x_{n}\right\}_{n \in \mathbb{N}}$ es p-convergente a $x_{0}$.

Por otro lado, por construcción, $x_{n} \in X \backslash B\left(x_{0}, r, t\right)$ para todo $n \in \mathbb{N}$, y así $\left\{x_{n}\right\}_{n \in \mathbb{N}}$ no converge a $x_{0}$, y por el Corolario $3.2 .3,\left\{x_{n}\right\}_{n \in \mathbb{N}}$ no es convergente.

Los siguientes ejemplos ilustran el último teorema.

Ejemplo 3.2.10. Sea $\left.\varphi: \mathbb{R}^{+} \longrightarrow\right] 0,1[$ una función continua y creciente. Definimos la función $M$ sobre $X^{2} \times \mathbb{R}^{+}$por

$$
M(x, y, t)= \begin{cases}1, & \text { si } x=y \\ \varphi(t), & \text { si } x \neq y\end{cases}
$$


Según la Proposición 2.4.1, $(M, \cdot)$ es una métrica fuzzy sobre $X$.

Ahora, para cada $x \in X$ y $t>0$ tenemos que $B(x, 1-\varphi(t), t)=\{x\}$ y asi $M$ es principal. Además, $\tau_{M}$ es la topología discreta, y entonces solamente las sucesiones constantes son convergentes, y por lo tanto son las únicas sucesiones p-convergentes en $X$.

A continuación damos un ejemplo de espacio métrico fuzzy completo que no es principal.

Ejemplo 3.2.11. Sea $X=\mathbb{R}^{+}$y sea $\left.\left.\varphi: \mathbb{R}^{+} \longrightarrow\right] 0,1\right]$ una función dada por $\varphi(t)=t$ si $t \leq 1$ y $\varphi(t)=1$ en cualquier otro caso. Definimos la función $M$ sobre $X^{2} \times \mathbb{R}^{+}$por

$$
M(x, y, t)=\left\{\begin{array}{lr}
1, & \text { si } x=y \\
\frac{\min \{x, y\}}{\max \{x, y\}} \cdot \varphi(t), & \text { si } x \neq y
\end{array}\right.
$$

Según la Proposición 2.8.2, se tiene que $(M, \cdot)$ es una métrica fuzzy sobre $X$.

Como $M(x, y, t)<t$, siempre que $t \in] 0,1[y \neq y$, es obvio que las únicas sucesiones de Cauchy en $X$ son las sucesiones constantes y asi, $X$ es completo.

Esta métrica fuzzy no es principal. En efecto, observemos que $B\left(x, \frac{1}{2}, \frac{1}{2}\right)=$ $\{x\}$ para cada $x \in X$ y asi $\tau_{M}$ es la topología discreta. Ahora, si $x=$ 1 y $t=1$ tenemos que $B(1, r, 1)=] 1-r, \frac{1}{1-r}[$ para todo $r \in] 0,1[y$ asi $\{B(1, r, 1): r \in] 0,1[\}$ no es una base local de $x=1$, ya que $\{1\}$ es abierto.

Ahora, consideremos la sucesión $\left\{x_{n}\right\}_{n \in \mathbb{N}}$ en $X$ dada por $x_{n}=1-$ $\frac{1}{n}, n \in \mathbb{N}$. Tomemos $M\left(x_{n}, 1,1\right)=1-\frac{1}{n}$ para todo $n \in \mathbb{N}$, por tanto 
$\lim _{n \rightarrow \infty} M\left(x_{n}, 1,1\right)=1 y\left\{x_{n}\right\}_{n \in \mathbb{N}}$ es p-convergente al punto 1 , pero no es convergente.

En el siguiente ejemplo, $(X, M)$ es un espacio métrico fuzzy que no es principal ni completable.

Ejemplo 3.2.12. Sea $X=] 0,1], A=X \cap \mathbb{Q}, B=X \backslash A$. Definimos la función $M$ sobre $X^{2} \times \mathbb{R}^{+}$por

$$
M(x, y, t)= \begin{cases}\frac{\min \{x, y\}}{\max \{x, y\}}, & \text { si } x, y \in A \text { ó } x, y \in B, t>0, \\ \frac{\min \{x, y\}}{\max \{x, y\}}, & \text { si } x \in A, y \in B \text { ó } x \in B, y \in A, t \geq 1 \\ \frac{\min \{x, y\}}{\max \{x, y\}} \cdot t, & \text { en otro caso }\end{cases}
$$

Veamos que $(M, \cdot)$ es una métrica fuzzy sobre $X$.

Para ello vamos a probar únicamente la desigualdad triangular, y lo haremos sólo para el caso $x \in A, z \in B$ (ó $x \in B, z \in A$ ) con $t+s<1$.

Se tiene que:

$$
\begin{gathered}
M(x, z, t+s)=\frac{\min \{x, z\}}{\max \{x, z\}} \cdot(t+s) \geq \frac{\min \{x, y\}}{\max \{x, y\}} \cdot \frac{\min \{y, z\}}{\max \{y, z\}} \cdot(t+s) \geq \\
\geq \frac{\min \{x, y\}}{\max \{x, y\}} \cdot t \cdot \frac{\min \{y, z\}}{\max \{y, z\}} \cdot s=M(x, y, t) \cdot M(y, z, s) .
\end{gathered}
$$

Las demás situaciones son obvias.

Ahora veremos que $M$ no es principal. Para ello veremos que

$$
\{B(1, r, 1): r \in] 0,1[\}
$$

no es una base local de $x=1$. 
En efecto, $B(1, r, 1)=] 1-r, 1]$ para todo $r \in] 0,1[$. Ahora, como $1 \in A$ tenemos que $\left\{y \in B: M\left(1, y, \frac{1}{2}\right)>1-\frac{1}{2}\right\}=\phi$, asi que $\left.\left.B\left(1, \frac{1}{2}, \frac{1}{2}\right)=\right] \frac{1}{2}, 1\right] \cap \mathbb{Q}$ y claramente $B(1, r, 1) \nsubseteq B\left(1, \frac{1}{2}, \frac{1}{2}\right)$ para todo $\left.r \in\right] 0,1[$, y entonces $M$ no es principal.

Ahora es fácil probar que para $n \geq 2$,

$$
B\left(x, \frac{1}{n}, \frac{1}{n}\right)= \begin{cases}] x-\frac{x}{n}, \frac{n x}{n-1}[\cap A, & \text { si } x \in A, \\ ] x-\frac{x}{n}, \frac{n x}{n-1}[\cap B, & \text { si } x \in B,\end{cases}
$$

y claramente $\tau_{M}$ no es la topología discreta, aunque es más fina que la topología usual de $\mathbb{R}$ relativa a $X$.

Finalmente sea $\left\{x_{n}\right\}_{n \in \mathbb{N}}$ una sucesión creciente contenida en $B$ que converge a 1 con la topología usual de $\mathbb{R}$ relativa a $X$. Así, para cada $r \in] 0,1[$ existe $n_{1} \in \mathbb{N}$ tal que $\left.x_{n} \in\right] 1-r, 1\left[\right.$ para todo $n \geq n_{1}$, y por la definición de $M$ tenemos que $M\left(x_{n}, 1,1\right)>1-r$, para todo $n \geq n_{1}$, y asi $\lim _{n \rightarrow \infty} M\left(x_{n}, 1,1\right)=1$, ya que $r$ es arbitrario. Entonces, $\left\{x_{n}\right\}_{n \in \mathbb{N}}$ es p-convergente a 1. Además, $\left\{x_{n}\right\}_{n \in \mathbb{N}}$ es una sucesión de Cauchy. Es más, como

$$
M\left(x_{n}, x_{m}, t\right)=\frac{\min \left\{x_{n}, x_{m}\right\}}{\max \left\{x_{n}, x_{m}\right\}} \text { para todo } t>0,
$$

tenemos que $M\left(x_{n}, x_{m}, t\right) \geq x_{n_{1}}>1-r$, para todo $t>0$, y asi $\left\{x_{n}\right\}_{n \in \mathbb{N}}$ es una sucesión de Cauchy. Ahora, $\left\{x_{n}\right\}_{n \in \mathbb{N}}$ no es convergente ya que $x_{n} \notin$ $B\left(1, \frac{1}{2}, \frac{1}{2}\right)$, para todo $n \in \mathbb{N}$ y entonces por el Corolario 3.2.4, $(X, M)$ no es completable.

Continuando el estudio iniciado por D. Mihet damos la siguiente definición.

Definición 3.2.13. Sea $(X, M, *)$ un espacio métrico fuzzy. Una sucesión $\left\{x_{n}\right\}_{n \in \mathbb{N}}$ en $X$ se dice que es $p$-Cauchy si para cada $\left.\varepsilon \in\right] 0,1\left[\right.$ existen $n_{0} \in \mathbb{N}$ 
y $t_{0}>0$ tales que $M\left(x_{n}, x_{m}, t_{0}\right)>1-\varepsilon$ para todo $n, m \geq n_{0}$, es decir, $\lim _{n, m \rightarrow \infty} M\left(x_{n}, x_{m}, t_{0}\right)=1$ para algún $t_{0}>0$.

En este caso decimos que $\left\{x_{n}\right\}_{n \in \mathbb{N}}$ es p-Cauchy para $t_{0}>0$, o simplemente, que $\left\{x_{n}\right\}_{n \in \mathbb{N}}$ es p-Cauchy.

Claramente $\left\{x_{n}\right\}_{n \in \mathbb{N}}$ es una sucesión de Cauchy si y sólo si $\left\{x_{n}\right\}_{n \in \mathbb{N}}$ es $p$-Cauchy para todo $t>0$, y obviamente, las sucesiones p-convergentes son p-Cauchy.

Definición 3.2.14. Un espacio métrico fuzzy $(X, M, *)$ se llama p-completo si toda sucesión p-Cauchy en $X$ es p-convergente a algún punto de $X$. En tal caso $M$ se llama p-completa.

Obviamente, p-completitud y completitud son conceptos equivalentes en métricas fuzzy estacionarias, y es fácil ver que la métrica fuzzy estándar $M_{d}$ es p-completa si y sólo si $M_{d}$ es completa como muestra la siguiente proposición.

Proposición 3.2.15. Sea $(X, d)$ un espacio métrico. Entonces $\left(X, M_{d}\right)$ es completo si y sólo si $\left(X, M_{d}\right)$ es p-completo.

\section{Demostración.}

Si $\left(X, M_{d}\right)$ es completo, evidentemente $\left(X, M_{d}\right)$ es p-completo. Veamos el recíproco.

Supongamos que $\left(X, M_{d}\right)$ es p-completo. Sea $\left\{x_{n}\right\}_{n \in \mathbb{N}}$ una sucesión de Cauchy en $\left(X, M_{d}\right)$. Entonces $\left\{x_{n}\right\}_{n \in \mathbb{N}}$ es p-Cauchy para todo $t>0$. Como $\left(X, M_{d}\right)$ es p-completo, $\left\{x_{n}\right\}_{n \in \mathbb{N}}$ es p-convergente en $X$. 
Supongamos que $\lim _{n \rightarrow \infty} M_{d}\left(x_{n}, x_{0}, t_{0}\right)=1$ para algún $x_{0} \in X$ y $t_{0}>0$. Entonces

$$
\lim _{n \rightarrow \infty} \frac{t_{0}}{t_{0}+d\left(x_{n}, x_{0}\right)}=1
$$

y por lo tanto $\lim _{n \rightarrow \infty} d\left(x_{n}, x_{0}\right)=0$, y en consecuencia

$$
\lim _{n \rightarrow \infty} \frac{t}{t+d\left(x_{n}, x_{0}\right)}=1 \text { para cualquier } t>0,
$$

y así $\left\{x_{n}\right\}_{n \in \mathbb{N}}$ converge a $x_{0}$.

Proposición 3.2.16. Sea $(X, M, *)$ un espacio métrico fuzzy principal. Si $X$ es $p$-completo entonces $X$ es completo.

\section{Demostración.}

Sea $\left\{x_{n}\right\}_{n \in \mathbb{N}}$ una sucesión de Cauchy en $X$. Entonces $\left\{x_{n}\right\}_{n \in \mathbb{N}}$ es pCauchy y así $\left\{x_{n}\right\}_{n \in \mathbb{N}}$ p-converge a algún punto $x_{0} \in X$, y como $X$ es principal, por el Teorema 3.2.9 tenemos que $\left\{x_{n}\right\}_{n \in \mathbb{N}}$ converge a $x_{0}$.

La hipótesis de que $X$ es principal no puede ser suprimida en la proposición anterior como vemos en el siguiente ejemplo.

Ejemplo 3.2.17. Consideremos el espacio métrico fuzzy $(X, M, *)$ del Ejemplo 3.2.5. La sucesión $\left\{x_{n}\right\}_{n \in \mathbb{N}}$ cumple que $\lim _{n, m \rightarrow \infty} M\left(x_{n}, x_{m}, t\right)=1$ para todo $t>0$, asi $\left\{x_{n}\right\}_{n \in \mathbb{N}}$ es una sucesión de Cauchy y como consecuencia $X$ no es completo, ya que $\left\{x_{n}\right\}_{n \in \mathbb{N}}$ no es convergente.

\section{Veamos que $X$ es p-completo.}

Sea $\left\{x_{n}\right\}_{n \in \mathbb{N}}$ una sucesión p-Cauchy en $X$. Entonces, con un fácil argumento, se puede verificar que $\left\{x_{n}\right\}_{n \in \mathbb{N}}$ debe ser una sucesión convergente a 1 con respecto a la topología usual de $\mathbb{R}$ relativa a $X$. Ahora, $\lim _{n \rightarrow \infty} M\left(x_{n}, 1,1\right)=$ $\lim _{n \rightarrow \infty} x_{n}=1$ y por lo tanto $\left\{x_{n}\right\}_{n \in \mathbb{N}}$ es p-convergente a 1 . 
Uno podría esperar que las sucesiones p-Cauchy sean sucesiones de Cauchy en espacios métricos fuzzy principales. En efecto, esta propiedad la satisfacen todos los ejemplos del Ejemplo 3.2.8. No obstante, como muestra el siguiente ejemplo, no se cumple en general, para cualquier espacio métrico fuzzy principal y, como consecuencia, no se cumple el recíproco de la proposición anterior.

Ejemplo 3.2.18. Sea $X=] 0,1\left[y\right.$ definimos la función $M$ sobre $X^{2} \times \mathbb{R}^{+}$ por

$$
M(x, y, t)= \begin{cases}1, & \text { si } x=y \\ x y t, & \text { si } x \neq y, t \leq 1 \\ x y, & \text { si } x \neq y, t>1\end{cases}
$$

Según la Proposición 2.8.2, $(M, \cdot)$ es una métrica fuzzy sobre $X$. Ahora, sea $x \in X y t>0$. Si tomamos $r \in] 0,1[$ tal que $1-r>x$ entonces $B(x, r, t)=\{x\}$ y asi $M$ es principal. Además $\tau_{M}$ es la topología discreta y entonces las únicas sucesiones convergentes o (por el Teorema 3.2.9) sucesiones p-convergentes, son las sucesiones constantes.

Ahora, $X$ no tiene sucesiones de Cauchy distintas de las constantes, ya que $M\left(x, y, \frac{1}{2}\right) \leq \frac{1}{2}$ para cada $x, y \in X$, y asi $X$ es completo.

Sea $\left\{x_{n}\right\}_{n \in \mathbb{N}}$ una sucesión estrictamente creciente y convergente a 1 en la topología usual de $\mathbb{R}$, relativa a $X$. Tenemos que $\lim _{n, m \rightarrow \infty} M\left(x_{n}, x_{m}, 1\right)=1$ $y$ entonces $\left\{x_{n}\right\}_{n \in \mathbb{N}}$ es $p$-Cauchy. Sin embargo $\left\{x_{n}\right\}_{n \in \mathbb{N}}$ no es p-convergente ya que $\left\{x_{n}\right\}_{n \in \mathbb{N}}$ no es constante, y asi $X$ no es p-completo.

Es un problema abierto caracterizar aquellos espacios métricos fuzzy donde la familia de sucesiones p-Cauchy y sucesiones de Cauchy coinciden, o más aún, cuándo se cumple que completitud es equivalente a p-completitud. 


\subsection{Continuidad en espacios métricos fuzzy}

Recordemos el concepto de aplicación t-uniformemente continua que podemos ver con más detalle en [24] y [28].

Definición 3.3.1. Sean $(X, M, *)$ e $(Y, N, \star)$ dos espacios métricos fuzzy. Se dice que la aplicación $f: X \rightarrow Y$ es t-uniformemente continua, si para todo $\varepsilon \in] 0,1[, t>0$, existe $\delta \in] 0,1[$ tal que si $M(x, y, t)>1-\delta$ entonces $N(f(x), f(y), t)>1-\varepsilon$, para cualesquiera $x, y \in X$.

La definición de continuidad de una aplicación $f$ de un espacio métrico fuzzy $(X, M)$ con valores en otro espacio métrico fuzzy $(Y, N)$ puede darse usando cuatro parámetros como vemos a continuación.

Una aplicación $f: X \rightarrow Y$ es continua en $x_{0} \in X$ si dado $\left.\varepsilon \in\right] 0,1[\mathrm{y}$ $t>0$ existen $\delta \in] 0,1\left[\mathrm{y} s>0\right.$ tales que si $M\left(x_{0}, x, s\right)>1-\delta$ entonces $N\left(f\left(x_{0}\right), f(x), t\right)>1-\varepsilon$.

Obviamente la condición de continuidad de una función $f$ entre espacios métricos fuzzy estacionarios solamente necesita dos parámetros. Entonces, pensando en espacios métricos fuzzy estacionarios y acorde con el concepto de t-uniformemente continua, damos la siguiente definición por medio de tres parámetros.

Definición 3.3.2. Diremos que una aplicación entre espacios métricos fuzzy $f:(X, M) \longrightarrow(Y, N)$ es t-continua en $x_{0} \in X$ si dados $\left.\varepsilon \in\right] 0,1[y t>0$ existe $\delta \in] 0,1\left[\right.$ tal que si $M\left(x_{0}, x, t\right)>1-\delta$, entonces $N\left(f\left(x_{0}\right), f(x), t\right)>$ $1-\varepsilon$.

Definición 3.3.3. Diremos que la aplicación $f:(X, M) \longrightarrow(Y, N)$ es $t$ continua en $X$ sif es t-continua en cada $x \in X$. 
Si $M$ es una métrica fuzzy estacionaria, resulta evidente que toda aplicación continua en $x_{0} \in X$ es t-continua en $x_{0}$.

Si $f$ es t-continua en $x_{0}$, obviamente $f$ es continua en $x_{0}$. El recíproco es falso como mostramos en el siguiente ejemplo.

Ejemplo 3.3.4. Sea $\left\{x_{n}\right\}_{n \in \mathbb{N}}$ una sucesión estrictamente creciente y convergente a 1 en la topología usual de $\mathbb{R}$. Sea $X=\left\{x_{n}\right\} \cup\{1\}$. Para cada $x, y \in X$ y $t>0$ definimos la función $M$ sobre $X^{2} \times \mathbb{R}^{+}$dada por

$$
M(x, y, t)= \begin{cases}1, & \text { si } x=y \\ t x y, & \text { si } x \neq y, t<1 \\ x y, & \text { si } x \neq y, t \geq 1\end{cases}
$$

Según el Ejemplo 2.8.4, $(M, \cdot)$ es una métrica fuzzy sobre $X$. Además, para cada $x \in X$ tenemos que $B\left(x, \frac{1}{2}, \frac{1}{2}\right)=\{x\}$ y entonces $\tau_{M}$ es la topología discreta sobre $X$.

Consideremos la función $f:(X, M) \rightarrow(X, M)$ dada por

$$
f(x)= \begin{cases}\frac{1}{2}, & \text { si } x \neq 1 \\ 1, & \text { si } x=1\end{cases}
$$

Obviamente $f$ es continua en $X$. Veamos que $f$ no es t-continua en $x_{0}=1$. En efecto, sea $t=1$ y $\varepsilon=0.1$. Para cada $\delta>0$ podemos encontrar $x_{m} \in X$ tal que $1-\delta<x_{m}<1$ y entonces $M\left(1, x_{m}, 1\right)=x_{m}>1-\delta$. Como $f\left(x_{m}\right)=0.5$ entonces $M\left(f(1), f\left(x_{m}\right), 1\right)=M\left(1, \frac{1}{2}, 1\right)=0.5 \leq 1-0.1$ y entonces $f$ no es $t$-continua en $x_{0}=1$.

Es obvio que cada función t-uniformemente continua es t-continua. El recíproco es falso. Veremos en el siguiente ejemplo una función t-continua (y uniformemente continua) que no es t-uniformemente continua. 
Ejemplo 3.3.5. Sea $X=\{1,2,3, \ldots\}$. Consideremos sobre $X$ la métrica fuzzy $M$ dada por

$$
M(m, n, t)= \begin{cases}\frac{\min \{m, n\}}{\max \{m, n\}} \cdot t, & \text { si } m \neq n, t<1 \\ \frac{\min \{m, n\}}{\max \{m, n\}}, & \text { en los demás casos }\end{cases}
$$

donde la t-norma $*$ es el producto usual en $[0,1]$.

Para cada $n \in X$ tenemos que $B\left(n, \frac{1}{2}, \frac{1}{2}\right)=\{n\}$ y por tanto $\tau_{M}$ es la topología discreta en $X$.

Veamos que toda función $f: X \longrightarrow \mathbb{R}$ es uniformemente continua para cualquier métrica fuzzy $N$ sobre $\mathbb{R}$. En efecto, sean $t>0$ y $\varepsilon \in] 0,1[$. Consideremos $s=\delta=0.5$. Se tiene que $M(n, m, s)>1-\delta$ si y sólo si $n=m, y$ por lo tanto si $M(n, m, s)>1-\delta$, entonces $N(f(n), f(m), t)=1(>1-\varepsilon)$.

Veamos ahora que toda función $f: X \longrightarrow \mathbb{R}$ es t-continua para cualquier métrica fuzzy $N$ sobre $\mathbb{R}$. En efecto, sean $t>0, \varepsilon \in] 0,1[y$ sea $n \in X$. Consideremos $0<\delta<1-\frac{n}{n+1}$. Se tiene que

$$
M(n, m, t)>1-\delta>1-\left(1-\frac{n}{n+1}\right)=\frac{n}{n+1} \text { si y sólo si } n=m .
$$

Por tanto, si $M(n, m, t)>1-\delta$, entonces

$$
N(f(n), f(m), t)=1(>1-\varepsilon),
$$

$y$ asi $f$ es t-continua en $n$.

Ahora, consideremos la aplicación $f: X \longrightarrow \mathbb{R}$ definida por

$$
f(x)= \begin{cases}1, & \text { si } x \text { es impar } \\ 0, & \text { si } x \text { es } \text { par }\end{cases}
$$


Consideremos la métrica fuzzy $M$ sobre $X$, y la estándar $M_{|\cdot|}$ sobre $\mathbb{R}$, siendo $|\cdot|$ la métrica usual sobre $\mathbb{R}$. Veamos que $f$ no es t-uniformemente continua para las métricas fuzzy consideradas.

Sean $t=1, \varepsilon=0,5$. Para cualquier $\delta \in] 0,1[$ existe $n \in X$ tal que $\frac{n}{n+1}>1-\delta$, y por lo tanto $M(n, n+1, t)=\frac{n}{n+1}>1-\delta$, y se tiene que $M_{|\cdot|}(f(n), f(n+1), t)=\frac{1}{1+1}=\frac{1}{2}$, con lo que $f$ no es t-uniformemente continua.

La siguiente proposición muestra una clase más grande que las métricas fuzzy estacionarias en la cual las funciones continuas son t-continuas.

Proposición 3.3.6. Sea $f:(X, M) \rightarrow(Y, N)$ una función entre espacios métricos fuzzy tal que $f$ es continua en $x_{0}$. Si $M$ es una métrica fuzzy principal, entonces $f$ es t-continua en $x_{0}$.

\section{Demostración.}

Sean $x_{0} \in X, t>0$ y $\left.\varepsilon \in\right] 0,1[$.

Como $f$ es continua en $x_{0} \in X$, existen $\left.\delta^{\prime} \in\right] 0,1[$ y $s>0$ tales que $f\left(B\left(x_{0}, \delta^{\prime}, s\right)\right) \subset B\left(f\left(x_{0}\right), \varepsilon, t\right)$, y como $M$ es principal, existe $\left.\delta \in\right] 0,1[$ tal que $B\left(x_{0}, \delta, t\right) \subset B\left(x_{0}, \delta^{\prime}, s\right)$, y por lo tanto

$$
f\left(B\left(x_{0}, \delta, t\right)\right) \subset B\left(f\left(x_{0}\right), \varepsilon, t\right),
$$

es decir, $M\left(x_{0}, x, t\right)>1-\delta$ implica que $N\left(f\left(x_{0}\right), f(x), t\right)>1-\varepsilon$.

Si $(X, d)$ es un espacio métrico entonces la métrica fuzzy estándar $M_{d}$ es principal, como podemos ver en el Ejemplo 3.2.8 (b), y por lo tanto tenemos el siguiente corolario. 
Corolario 3.3.7. Una función $f$ de un espacio métrico fuzzy $\left(X, M_{d}\right)$ con valores en otro espacio métrico fuzzy $(Y, N)$ es continua en $x_{0}$ si y sólo si $f$ es $t$-continua en $x_{0}$.

Demostración.

Es obvio pues $M_{d}$ es principal. 


\section{Capítulo 4}

\section{Métricas fuzzy fuertes}

\subsection{Introducción}

Las ultramétricas fuzzy (métricas fuzzy con la t-norma del mínimo que verifican que $M(x, z, t) \geq M(x, y, t) \wedge M(y, z, t))$ y las métricas fuzzy estacionarias, en el sentido de George y Veeramani, no admiten completación en general. En este capítulo encontramos una clase de métricas fuzzy estacionarias completables que incluye a la clase de las ultrametricas fuzzy estacionarias. En la primera parte del capítulo, definimos y estudiamos algunos aspectos de las métricas fuzzy fuertes. En particular, se estudia la completitud de esta clase de métricas fuzzy. Para ello comenzamos definiendo las métricas fuzzy fuertes en la Definición 4.2.1. Una métrica fuzzy $M$ se dice fuerte si para cada $x, y, z \in X, t>0$ cumple que

$$
M(x, z, t) \geq M(x, y, t) * M(y, z, t) \quad\left(G V 4^{\prime}\right) .
$$


A continuación, el Ejemplo 4.2.2 nos hace ver que la condición de métrica fuerte no puede reemplazar al axioma $(G V 4)$ en la definición de métrica fuzzy, pero es posible definir una métrica fuzzy fuerte reemplazando $(G V 4)$ por $\left(G V 4^{\prime}\right)$ y exigiendo además en $(G V 5)$ que la función $M_{x y}$ sea además de continua, creciente respecto de $t$, para cada $x, y \in X$.

Si $(X, M, *)$ es un espacio métrico fuzzy no estacionario, definimos la familia de funciones $\left\{M_{t}: t \in \mathbb{R}^{+}\right\}$, donde para cada $\left.\left.t \in \mathbb{R}^{+}, M_{t}: X^{2} \rightarrow\right] 0,1\right]$ viene dada por $M_{t}(x, y)=M(x, y, t)$, que llamaremos familia de métricas fuzzy estacionarias asociadas a $M$. Así, en la Proposición 4.2.5 (i) podemos demostrar que $(M, *)$ es fuerte si y sólo si $\left(M_{t}, *\right)$ es una métrica fuzzy estacionaria sobre $X$ para cada $t \in \mathbb{R}^{+}$, y en (ii), que si $(M, *)$ es fuerte entonces la topología asociada a $M$ viene dada por $\tau_{M}=\bigvee\left\{\tau_{M_{t}}: t \in \mathbb{R}^{+}\right\}$. Además, la Proposición 4.2.7 muestra cuándo una familia $\left\{\left(M_{t}, *\right): t>0\right\}$ de métricas fuzzy estacionarias sobre $X$ definen una métrica fuzzy $(M, *)$ sobre $X$ mediante la fórmula $M(x, y, t)=M_{t}(x, y)$ para cada $x, y, \in X, t>0$, y esto ocurre si y sólo si la familia $\left\{M_{t}: t \in \mathbb{R}^{+}\right\}$es creciente (i.e $M_{t} \leq M_{s}$ si $t<s$ ) y la función $\left.\left.M_{x y}: \mathbb{R}^{+} \rightarrow\right] 0,1\right]$ dada por $M_{x y}(t)=M_{t}(x, y)$ es una función continua sobre $\mathbb{R}^{+}$, para cada $x, y \in X$. Finalmente, en el Corolario 4.2.10 podemos ver que un espacio métrico fuzzy fuerte $(X, M, *)$ es completo si y sólo si $\left(X, M_{t}, *\right)$ es completo para todo $t \in \mathbb{R}^{+}$, después de que en la Proposición 4.2.9, hayamos caracterizado las sucesiones que son $M_{t}$-Cauchy.

En la segunda parte estudiamos la completación de métricas fuzzy fuertes. Comenzamos definiendo (Definición 4.2.12) una t-norma continua $*$ íntegra como aquella que verifica que $a * b \neq 0$ siempre que $a, b \neq 0$. Obviamente las t-normas continuas mínimo y producto usual son íntegras. Tras demostrar el Teorema 4.2.14, (que dice que en un espacio métrico fuzzy fuerte $(X, M, *)$ con $*$ íntegra, si $\left\{x_{n}\right\}_{n \in \mathbb{N}}$ e $\left\{y_{n}\right\}_{n \in \mathbb{N}}$ son sucesiones de Cauchy en $X$ y $t>0$, entonces la sucesión $\left\{M\left(x_{n}, y_{n}, t\right)\right\}_{n \in \mathbb{N}}$ converge en $\left.\left.] 0,1\right]\right)$ obtenemos como 
consecuencia el Corolario 4.2.15 donde vemos que si $(M, *)$ es una métrica fuzzy estacionaria sobre $X$ y $*$ es íntegra entonces $(X, M, *)$ es completable. Como consecuencia (Corolario 4.2.16), las ultramétricas fuzzy estacionarias son completables. Más adelante, el Teorema 4.2.18 demuestra que si $(X, M, *)$ es un espacio métrico fuzzy fuerte completable, entonces $\left(X, M_{t}, *\right)$ es completable para todo $t>0$, y en el Ejemplo 4.2 .19 vemos que el recíproco es falso y además que el Corolario 4.2.16 no se puede extender a ultramétricas fuzzy. Como resultado final tenemos el Teorema 4.2.20, que afirma que un espacio métrico fuzzy fuerte $(X, M, *)$ donde $*$ es íntegra, la topología asociada a $M, \tau_{M}$, es el supremo de una familia decreciente de topologías fuzzy metrizables estacionarias las cuales son completables.

\subsection{Una clase de espacios métricos fuzzy comple- tables}

\subsubsection{Métricas fuzzy fuertes. Completitud}

$\operatorname{Si}(M, \wedge)$ es una métrica fuzzy sobre $X$ entonces la desigualdad triangular $(G V 4)$ se convierte para todo $x, y, z \in X$ y $t, s>0$ en

$$
M(x, z, t+s) \geq M(x, y, t) \wedge M(y, z, s) .
$$

Ahora, si exigimos también que

$$
M(x, z, t) \geq M(x, y, t) \wedge M(y, z, t)
$$

obtenemos la noción de ultramétrica fuzzy (o métrica fuzzy no arquimediana [49]), lo que sugiere la siguiente definición. 
Definición 4.2.1. Sea $(X, M, *)$ un espacio métrico fuzzy. La métrica fuzzy $M$ se dice fuerte si para cada $x, y, z \in X$ y $t>0$ cumple que

$$
M(x, z, t) \geq M(x, y, t) * M(y, z, t) \quad\left(G V 4^{\prime}\right)
$$

Observar que este axioma $\left(G V 4^{\prime}\right)$ no puede reemplazar al axioma $(G V 4)$, en la definición de métrica fuzzy, cuando pretendemos obtener una métrica fuzzy, ya que en este caso $M$ podría no ser una métrica fuzzy sobre $X$ como podemos ver en el siguiente ejemplo.

Ejemplo 4.2.2. Consideremos la métrica usual $|\cdot|$ sobre $\mathbb{R}$. Definimos la función $\left.\left.M: \mathbb{R} \times \mathbb{R} \times \mathbb{R}^{+} \rightarrow\right] 0,1\right]$ por

$$
M(x, y, t)=\frac{\frac{1}{t}}{\frac{1}{t}+|x-y|}
$$

Entonces $M$ cumple $(G V 1)-(G V 3)$ y $(G V 5)$ pero no satisface $(G V 4)$ para el producto usual. En efecto, sean $x=1, y=2, z=3, t=s=1$. Entonces tenemos

$M(x, z, t+s)=\frac{\frac{1}{2}}{\frac{1}{2}+|3-1|}<\frac{1}{1+|2-1|} \cdot \frac{1}{1+|3-2|}=M(x, y, t) \cdot M(y, z, s)$

Observar que la función $M_{x y}$ es estrictamente decreciente para todo $t>0$.

Sin embargo $M$ satisface $\left(G V 4^{\prime}\right)$ para el producto usual. En efecto,

$$
\begin{aligned}
& M(x, z, t)=\frac{\frac{1}{t}}{\frac{1}{t}+|z-x|} \geq \frac{\frac{1}{t}}{\frac{1}{t}+|y-x|+|z-y|} \geq \\
& \geq \frac{\frac{1}{t}}{\frac{1}{t}+|y-x|} \cdot \frac{\frac{1}{t}}{\frac{1}{t}+|z-y|}=M(x, y, t) \cdot M(y, z, t)
\end{aligned}
$$

Equivalentemente, es posible definir una métrica fuzzy fuerte reemplazando $(G V 4)$ por $\left(G V 4^{\prime}\right)$ si exigimos además en $(G V 5)$ que la función $M_{x y}$ 
sea una función continua y creciente sobre $t$, para cada $x, y \in X$. En efecto, en tal caso tenemos que

$$
M(x, z, t+s) \geq M(x, y, t+s) * M(y, z, t+s) \geq M(x, y, t) * M(y, z, s) .
$$

\section{Ejemplo 4.2.3.}

(a) Las métricas fuzzy estacionarias son fuertes.

(b) Las ultramétricas fuzzy son fuertes.

(c) La métrica fuzzy $(M, \cdot)$ sobre $\mathbb{R}^{+}$definida por

$$
M(x, y, t)=\frac{\min \{x, y\}+\varphi(t)}{\max \{x, y\}+\varphi(t)},
$$

donde $\varphi:] 0,+\infty[\rightarrow] 0,+\infty[$ es una aplicación creciente y continua, es fuerte.

Los dos siguientes ejemplos son (métricas fuzzy fuertes definidas por métricas fuzzy estacionarias).

(d) Sea $(N, \cdot)$ una métrica fuzzy estacionaria sobre $X$. Definimos la función $M$ sobre $X^{2} \times \mathbb{R}^{+}$por

$$
M(x, y, t)=\frac{t}{t+1-N(x, y)}
$$

Entonces $(M, \cdot)$ es una métrica fuzzy fuerte sobre $X$.

(e) Sea $(N, \cdot)$ una métrica fuzzy estacionaria sobre $X$. Definimos la función $M$ sobre $X^{2} \times \mathbb{R}^{+}$por

$$
M(x, y, t)=\frac{t+N(x, y)}{t+1}
$$

Entonces $(M, \cdot)$ es una métrica fuzzy fuerte sobre $X$. 
En los siguientes ejemplos d es una métrica sobre $X$.

(f) Sea $\left.\left.\varphi: \mathbb{R}^{+} \rightarrow\right] 0,1\right]$ una funcion continua creciente. Definimos la función $M$ sobre $X^{2} \times \mathbb{R}^{+}$por

$$
M(x, y, t)=\frac{\varphi(t)}{\varphi(t)+d(x, y)}
$$

Entonces $(M, \cdot)$ es fuerte. En particular, la métrica fuzzy estándar $M_{d}$ es fuerte para el producto usual.

(g) La función $M$ sobre $X^{2} \times \mathbb{R}^{+}$dada por

$$
M(x, y, t)=e^{-\frac{d(x, y)}{t}}
$$

es fuerte para el producto usual.

(h) La métrica fuzzy estándar $M_{d}$ es una ultramétrica fuzzy (si y sólo si es fuerte para la t-norma mínimo) si y sólo si d es una ultramétrica [49].

Por consiguiente,

(i) Si d es una métrica que no es ultramétrica entonces $\left(M_{d}, \wedge\right)$ es una métrica fuzzy no-fuerte sobre $X$.

Demostración.

(c) Veamos que para todo $x, y, z \in X, t>0$, se tiene que

$$
M(x, z, t) \geq M(x, y, t) * M(y, z, t) .
$$

Supongamos que $x<z$. Distinguimos tres casos: 
Caso 1. Supongamos que $x \leq y \leq z$. Se tiene entonces que

$$
M(x, z, t)=\frac{x+\varphi(t)}{z+\varphi(t)}=\frac{x+\varphi(t)}{y+\varphi(t)} \cdot \frac{y+\varphi(t)}{z+\varphi(t)}=M(x, y, t) * M(y, z, t) .
$$

Caso 2. Supongamos que $y \leq x<z$. Se tiene entonces que

$$
\begin{gathered}
M(x, z, t)=\frac{x+\varphi(t)}{z+\varphi(t)} \geq \frac{y+\varphi(t)}{z+\varphi(t)} \geq \\
\geq \frac{x+\varphi(t)}{y+\varphi(t)} \cdot \frac{y+\varphi(t)}{z+\varphi(t)}=M(x, y, t) * M(y, z, t) .
\end{gathered}
$$

Caso 3. Supongamos que $z \leq y$. Se tiene entonces que

$$
\begin{gathered}
M(x, z, t)=\frac{x+\varphi(t)}{z+\varphi(t)} \geq \frac{x+\varphi(t)}{y+\varphi(t)} \geq \\
\geq \frac{x+\varphi(t)}{y+\varphi(t)} \cdot \frac{z+\varphi(t)}{y+\varphi(t)}=M(x, y, t) * M(y, z, t) .
\end{gathered}
$$

Análogamente se prueba si $z<x$.

(d) Por la Proposición 2.8.5 sabemos que $(M, \cdot)$ es una métrica fuzzy sobre $X$. Sean $x, y, z \in X$ y $t>0$. Veamos que

$$
M(x, z, t) \geq M(x, y, t) \cdot M(y, z, t) .
$$

Probar la desigualdad anterior es equivalente a probar

$$
\frac{t}{t+1-N(x, z)} \geq \frac{t}{t+1-N(x, y)} \cdot \frac{t}{t+1-N(y, z)}
$$

que equivale a

$$
((t+1)-N(x, y)) \cdot((t+1)-N(y, z)) \geq t \cdot(t+1-N(x, z))
$$

que equivale sucesivamente a

$1+t \cdot(1+N(x, z))-t N(y, z)-t N(x, y)+N(x, y) \cdot N(y, z)-N(y, z)-N(x, y) \geq 0$ 
y también a

$t[(1+N(x, z)-N(y, z)-N(x, y)]+1+N(x, y) \cdot N(y, z)-N(y, z)-N(x, y) \geq 0$

lo cual es cierto ya que

$$
[1+N(x, z)-N(y, z)-N(x, y)]>0
$$

y

$1+N(x, y) \cdot N(y, z)-N(y, z)-N(x, y)=(1-N(x, y)) \cdot(1-N(y, z))>0$.

Luego $M$ es una métrica fuzzy fuerte.

(e) Por la Proposición 2.8.1 sabemos que $(M, \cdot)$ es una métrica fuzzy sobre $X$. Sean $x, y, z \in X$ y $t>0$. Veamos que

$$
M(x, z, t) \geq M(x, y, t) \cdot M(y, z, t) .
$$

Probar la desigualdad anterior es equivalente a probar

$$
\frac{t+N(x, z)}{t+1} \geq \frac{t+N(x, y)}{t+1} \cdot \frac{t+N(y, z)}{t+1}
$$

que equivale a

$$
(t+1) \cdot(t+N(x, z)) \geq(t+N(x, y)) \cdot(t+N(y, z))
$$

(sabiendo que $N(x, z) \geq N(x, y) \cdot N(y, z))$ que equivale sucesivamente a

$$
\begin{gathered}
t^{2}+t N(x, z)+t+N(x, z) \geq t^{2}+t N(y, z)+t N(x, y)+N(x, y) N(y, z) \\
t(N(x, z)+1) \geq t(N(y, z)+N(x, y)) \\
N(x, z)+1 \geq N(y, z)+N(x, y)
\end{gathered}
$$


y esta última se cumple, pues

$$
N(x, z)+1-N(y, z)-N(x, y) \geq
$$

$\geq N(x, y) \cdot N(y, z)+1-N(y, z)-N(x, y)=(1-N(x, y))(1-N(y, z))>0$.

Suponemos $x \neq y$ e $y \neq z$. Los demás casos son obvios.

Luego $M$ es una métrica fuzzy fuerte.

(f) Sean $x, y, z \in X, t>0$. Para ver

$$
M(x, z, t) \geq M(x, y, t) \cdot M(y, z, t),
$$

hemos de probar que

$$
\frac{\varphi(t)}{\varphi(t)+d(x, z)} \geq \frac{\varphi(t)}{\varphi(t)+d(x, y)} \cdot \frac{\varphi(t)}{\varphi(t)+d(y, z)}
$$

que es lo mismo que probar que

$$
(\varphi(t)+d(x, y)) \cdot(\varphi(t)+d(y, x)) \geq(\varphi(t)+d(x, z)) \cdot \varphi(t) .
$$

Pues bien

$$
\begin{gathered}
(\varphi(t)+d(x, y)) \cdot(\varphi(t)+d(y, x))= \\
=\varphi(t)^{2}+\varphi(t) \cdot d(x, y)+\varphi(t) \cdot d(y, z)+d(x, y) \cdot d(y, z)= \\
=\varphi(t)^{2}+\varphi(t) \cdot(d(x, y)+d(y, z))+d(x, y) \cdot d(y, z) \geq \\
\geq \varphi(t)^{2}+\varphi(t) \cdot d(x, z)+d(x, y) \cdot d(y, z) \geq \\
\geq \varphi(t)^{2}+\varphi(t) \cdot d(x, z)=\varphi(t)(\varphi(t)+d(x, z)) .
\end{gathered}
$$

(g) Sean $x, y, z \in X, t>0$. Para ver

$$
M(x, z, t) \geq M(x, y, t) \cdot M(y, z, t),
$$


tenemos que probar que

$$
e^{-\frac{d(x, z)}{t}} \geq e^{-\frac{d(x, y)}{t}} \cdot e^{-\frac{d(y, z)}{t}}
$$

Como $d$ es una métrica, se tiene que $d(x, z) \leq d(x, y)+d(y, z)$ (desigualdad triangular) lo que es equivalente a

$$
-\frac{d(x, z)}{t} \geq-\frac{d(x, y)}{t}-\frac{d(y, z)}{t}
$$

Por tanto

$$
e^{-\frac{d(x, z)}{t}} \geq e^{-\frac{d(x, y)}{t}-\frac{d(y, z)}{t}}
$$

lo cual es lo mismo que

$$
e^{-\frac{d(x, z)}{t}} \geq e^{-\frac{d(x, y)}{t}} \cdot e^{-\frac{d(y, z)}{t}}
$$

Problema 4.2.4. Encontrar una métrica fuzzy no-fuerte $(M, *)$ donde * es el producto usual o la t-norma de Lukasievicz.

Sea $(M, *)$ una métrica fuzzy no estacionaria sobre $X$. Definimos la familia de funciones $\left\{M_{t}: t \in \mathbb{R}^{+}\right\}$donde para cada $\left.\left.t \in \mathbb{R}^{+}, M_{t}: X^{2} \rightarrow\right] 0,1\right]$ viene dada por $M_{t}(x, y)=M(x, y, t)$. Con esta notación, tenemos la siguiente proposición.

Proposición 4.2.5. Sea $(M, *)$ una métrica fuzzy sobre $X$ no estacionaria. Entonces:

(i) $(M, *)$ es fuerte si y sólo si $\left(M_{t}, *\right)$ es una métrica fuzzy estacionaria sobre $X$ para cada $t \in \mathbb{R}^{+}$.

(ii) Si $(M, *)$ es fuerte entonces $\tau_{M}=\bigvee\left\{\tau_{M_{t}}: t \in \mathbb{R}^{+}\right\}$ 


\section{Demostración.}

(i) $\mathrm{Si}(M, *)$ es fuerte entonces es obvio que $\left(M_{t}, *\right)$ es una métrica fuzzy estacionaria sobre $X$ para cada $t \in \mathbb{R}^{+}$.

Veamos el recíproco. Sean $x, y, z \in X$ y $t>0$. Entonces

$$
M(x, z, t)=M_{t}(x, z) \geq M_{t}(x, y) * M_{t}(y, z)=M(x, y, t) * M(y, z, t)
$$

(ii) Es evidente pues para cada $x \in X, r \in] 0,1[\mathrm{y} t>0$, las bolas $B_{M}(x, r, t)$ coinciden con las bolas $B_{M_{t}}(x, r)$.

Si $M$ es una métrica fuzzy fuerte diremos que $\left\{M_{t}: t \in \mathbb{R}^{+}\right\}$es la familia de métricas fuzzy estacionarias asociadas a $M$.

\section{Ejemplo 4.2.6.}

(a) Sea d una métrica sobre $X$. Entonces $\left(\left(M_{d}\right)_{t}, \wedge\right)$ es una métrica fuzzy estacionaria sobre $X$ para cada $t>0$ si y sólo si $\left(M_{d}, \wedge\right)$ es fuerte si y sólo si d es una ultramétrica sobre $X$.

(b) Consideremos la métrica fuzzy fuerte $M$ sobre $\mathbb{R}^{+}$del apartado (c) del Ejemplo 4.2.3. Entonces,

$$
M_{t}(x, y)=\frac{\min \{x, y\}+t}{\max \{x, y\}+t}
$$

es una métrica fuzzy estacionaria para cada $t>0$ y es fácil verificar que $\tau_{M_{t}}=\tau_{M}$ para cada $t>0$.

(c) Consideremos la función $M$ sobre $\mathbb{R}^{+} \times \mathbb{R}^{+} \times \mathbb{R}^{+}$dada por

$$
M(x, y, t)= \begin{cases}1, & \text { si } x=y \\ \frac{\min \{x, y\}}{\max \{x, y\}} \cdot \varphi(t), & \text { si } x \neq y\end{cases}
$$


donde

$$
\varphi(t)= \begin{cases}t, & \text { si } 0<t \leq 1 \\ 1, & \text { si } t>1\end{cases}
$$

Según la Proposición 2.8.2, $(M, \cdot)$ es una métrica fuzzy sobre $\mathbb{R}^{+}$.

Veamos que $M$ es fuerte, es decir que $M(x, z, t) \geq M(x, y, t) \cdot M(y, z, t)$.

Sean $x, y, x \in \mathbb{R}^{+}, t>0$. Suponemos $x, y, z$ distintos (de lo contrario, es trivial). Entonces se tiene que:

$$
\begin{gathered}
M(x, z, t)=\frac{\min \{x, z\}}{\max \{x, z\}} \cdot \varphi(t) \geq \frac{\min \{x, y\}}{\max \{x, y\}} \cdot \frac{\min \{y, z\}}{\max \{y, z\}} \cdot \varphi(t) \geq \\
\geq \frac{\min \{x, y\}}{\max \{x, y\}} \cdot \varphi(t) \cdot \frac{\min \{y, z\}}{\max \{y, z\}} \cdot \varphi(t)=M(x, y, t) \cdot M(y, z, t) .
\end{gathered}
$$

Las demás situaciones son obvias.

Además, $\tau_{M}$ es la topología discreta sobre $\mathbb{R}^{+}$.

Para $t \geq 1$ tenemos que $M_{t}(x, t)=\frac{\min \{x, y\}}{\max \{x, y\}}$ y $\tau_{M_{t}}$ es la topología usual de $\mathbb{R}$ relativa $a \mathbb{R}^{+}$.

Para $t<1$ tenemos que $M_{t}(x, y)=\frac{\min \{x, y\}}{\max \{x, y\}} \cdot t$ y por lo tanto es fácil inferir que $\tau_{M_{t}}$ es la topología discreta.

Ahora surge la cuestión natural de cuándo una familia $\left\{\left(M_{t}, *\right): t>0\right\}$ de métricas fuzzy estacionarias sobre $X$ definen una métrica fuzzy $(M, *)$ sobre $X$ por medio de la fórmula $M(x, y, t)=M_{t}(x, y)$ para cada $x, y \in X$, $t \in \mathbb{R}^{+}$. La siguiente proposición contesta esta cuestión.

Proposición 4.2.7. Sea $\left\{\left(M_{t}, *\right): t \in \mathbb{R}^{+}\right\}$una familia de métricas fuzzy estacionarias sobre $X$. 
(i) La función $M$ sobre $X^{2} \times \mathbb{R}^{+}$definida por $M(x, y, t)=M_{t}(x, y)$ es una métrica fuzzy sobre $X$ cuando consideramos la t-norma $*$ si y sólo si la familia $\left\{M_{t}: t \in \mathbb{R}^{+}\right\}$es creciente (es decir, $M_{t} \leq M_{s}$ si $t<s$ ) y la función $\left.\left.M_{x y}: \mathbb{R}^{+} \rightarrow\right] 0,1\right]$ definida por $M_{x y}(t)=M_{t}(x, y)$ es una función continua sobre $\mathbb{R}^{+}$, para cada $x, y \in X$.

(ii) Si la familia $\left\{M_{t}: t>0\right\}$ satisface las dos condiciones de (i) entonces $(M, *)$ es fuerte $y\left\{\left(M_{t}, *\right): t \in \mathbb{R}^{+}\right\}$es la familia de métricas fuzzy estacionarias asociadas a $M$.

\section{Demostración.}

(i) Si $M$ es una métrica fuzzy sobre $X$, la conclusión es obvia.

Recíprocamente, supongamos que $\left\{M_{t}: t \in \mathbb{R}^{+}\right\}$verifica la condición del enunciado. Entonces para todo $x, y, z \in X, t, s>0$ se tiene

$$
\begin{gathered}
M(x, z, t+s)=M_{t+s}(x, z) \geq M_{t+s}(x, y) * M_{t+s}(y, z) \geq \\
\geq M_{t}(x, y) * M_{s}(y, z)=M(x, y, t) * M(y, z, s)
\end{gathered}
$$

Los demás axiomas $(G V 1)-(G V 3)$ y $(G V 5)$ se satisfacen obviamente.

(ii) Para todo $x, y, z \in X, t>0$ se tiene que

$$
M(x, z, t)=M_{t}(x, z) \geq M_{t}(x, y) * M_{t}(y, z)=M(x, y, t) * M(y, z, t)
$$

y por lo tanto $M$ es una métrica fuzzy fuerte.

Por (ii) podemos observar que una métrica fuzzy fuerte se caracteriza por ser asociada a la familia $\left\{M_{t}: t \in \mathbb{R}^{+}\right\}$de métricas fuzzy estacionarias.

El siguiente ejemplo ilustra la proposición anterior. 


\section{Ejemplo 4.2.8.}

(a) Consideremos sobre $\mathbb{R}$ la familia creciente de métricas fuzzy estacionarias $\left\{\left(M_{t}, \cdot\right): t \in \mathbb{R}^{+}\right\}$dada por

$$
M_{t}(x, y)=\frac{1}{1+|x-y|} \text { si } t \leq 1
$$

$y$

$$
M_{t}(x, y)=\frac{2}{2+|x-y|} \text { si } t>1
$$

Definimos la función $M$ sobre $\mathbb{R} \times \mathbb{R} \times \mathbb{R}^{+}$por $M(x, y, t)=M_{t}(x, y)$. Entonces $M_{x y}$ (definida para $x, y \in X$ por $M_{x y}(t)=M(x, y, t)$ para cada $t>0$ ) es una función creciente sobre $\mathbb{R}^{+}$pero $(M, \cdot)$ no es una métrica fuzzy sobre $\mathbb{R}$ ya que $M_{x y}$ no es continua para $t=1$ si $x \neq y$.

(b) Consideremos sobre $\mathbb{R}$ la familia de métricas fuzzy estacionarias $\left\{\left(M_{t}, \cdot\right): t \in \mathbb{R}^{+}\right\}$dada por

$$
M_{t}(x, y)=\frac{1}{1+|x-y|} \text { si } t \leq 1
$$

$y$

$$
M_{t}(x, y)=\frac{\frac{1}{t}}{\frac{1}{t}+|x-y|} \text { si } t>1 \text {. }
$$

Definimos la función $M$ sobre $\mathbb{R} \times \mathbb{R} \times \mathbb{R}^{+}$por $M(x, y, t)=M_{t}(x, y)$. En este caso $M_{x y}$, definida como antes, es una función continua sobre $\mathbb{R}^{+}$ pero $(M, \cdot)$ no es una métrica fuzzy sobre $\mathbb{R}$ ya que $M_{x y}$ no es una función creciente sobre $\mathbb{R}^{+}$.

Una consecuencia fácil de las definiciones previas es la siguiente proposición. 
Proposición 4.2.9. Sea $\left\{M_{t}: t \in \mathbb{R}^{+}\right\}$la familia de métricas fuzzy estacionarias asociadas a la métrica fuzzy fuerte $M$ en $X$. Entonces la sucesión $\left\{x_{n}\right\}_{n \in \mathbb{N}}$ en $X$ es M-Cauchy si y sólo si $\left\{x_{n}\right\}_{n \in \mathbb{N}}$ es $M_{t}-$ Cauchy para cada $t>0$.

Tenemos el siguiente corolario.

Corolario 4.2.10. Sea $(X, M, *)$ un espacio métrico fuzzy fuerte. $(X, M, *)$ es completo si y sólo si $\left(X, M_{t}, *\right)$ es completo para cada $t \in \mathbb{R}^{+}$.

\section{Demostración.}

Es obvio ya que $\tau_{M}=\bigvee\left\{\tau_{M_{t}}: t>0\right\}$.

Ejemplo 4.2.11. Consideremos la métrica fuzzy del Ejemplo 3.2.12, es decir, $X=] 0,1], A=X \cap \mathbb{Q}, B=X \backslash A$ y la función $M$ sobre $X^{2} \times \mathbb{R}^{+}$dada por

$$
M(x, y, t)= \begin{cases}\frac{\min \{x, y\}}{\max \{x, y\}}, & \text { si } x, y \in A \text { ó } x, y \in B, t>0, \\ \frac{\min \{x, y\}}{\max \{x, y\}}, & \text { si } x \in A, y \in B \text { ó } x \in B, y \in A, t \geq 1 \\ \frac{\min \{x, y\}}{\max \{x, y\}} \cdot t, & \text { en otro caso }\end{cases}
$$

Veamos que $(M, \cdot)$ es una métrica fuzzy fuerte sobre $X$.

Sólo estudiaremos los casos no triviales, y obviaremos el estudio de otros casos similares por simetría del razonamiento.

(1) Supongamos que $x, z \in A, y \in B, t<1$. Entonces

$$
\begin{aligned}
& M(x, z, t)=\frac{\min \{x, z\}}{\max \{x, z\}} \geq \frac{\min \{x, y\}}{\max \{x, y\}} \cdot \frac{\min \{y, z\}}{\max \{y, z\}} \geq \\
& \geq \frac{\min \{x, y\}}{\max \{x, y\}} \cdot t \cdot \frac{\min \{y, z\}}{\max \{y, z\}} \cdot t=M(x, y, t) \cdot M(y, z, t)
\end{aligned}
$$


(2) Supongamos que $x \in A, y, z \in B, t<1$. Entonces

$$
\begin{gathered}
M(x, z, t)=\frac{\min \{x, z\}}{\max \{x, z\}} \cdot t \geq \\
\geq \frac{\min \{x, y\}}{\max \{x, y\}} \cdot \frac{\min \{y, z\}}{\max \{y, z\}} \cdot t=M(x, y, t) \cdot M(y, z, t)
\end{gathered}
$$

Para $t=\frac{1}{2}$ y $x<y$ tenemos que

$$
M_{\frac{1}{2}}(x, y)= \begin{cases}\frac{x}{y}, & \text { si } x, y \in A \text { ó } x, y \in B \\ \frac{x}{2 y}, & \text { si } x \in A, y \in B \text { ó } x \in B, y \in A\end{cases}
$$

Sea $\left\{x_{n}\right\}_{n \in \mathbb{N}}$ una sucesión estrictamente creciente en $B$ que converge a 1 para la topología usual de $\mathbb{R}$. Entonces $\left\{x_{n}\right\}_{n \in \mathbb{N}}$ es $M_{\frac{1}{2}}-$ Cauchy ya que $\lim _{m, n \rightarrow \infty} M_{\frac{1}{2}}\left(x_{n}, x_{m}\right)=1$. Sin embargo $\left\{x_{n}\right\}_{n \in \mathbb{N}}$ no converge en $\left(X, M_{\frac{1}{2}}\right)$ a ningún $x<1$ ya que $\lim _{n \rightarrow \infty} M_{\frac{1}{2}}\left(x, x_{n}\right) \leq \lim _{n \rightarrow \infty} \frac{x}{x_{n}}=x<1$. Por otro lado, esta sucesión no converge en $\left(X, M_{\frac{1}{2}}\right)$ a 1 ya que $\lim _{n \rightarrow \infty} M_{\frac{1}{2}}\left(x_{n}, 1\right)=\lim _{n \rightarrow \infty} \frac{x_{n}}{2}=\frac{1}{2}$.

Así, $M_{\frac{1}{2}}$ no es completo $y$, como consecuencia, $M$ no es completo.

Además, en el Ejemplo 3.2.12 probamos que $M$ no es completo.

\subsubsection{Completación de métricas fuzzy fuertes}

Definición 4.2.12. Diremos que una t-norma continua $*$ es integra si cumple que $a * b \neq 0$ siempre que $a, b \neq 0$

Las t-normas continuas mínimo y producto usual son íntegras. La t-norma $\mathfrak{L}$ de Lukasievicz no es íntegra. 
En el Ejemplo 1 de [23], se dan dos sucesiones de Cauchy $\left\{x_{n}\right\}_{n}$ e $\left\{y_{n}\right\}_{n}$ en un espacio métrico fuzzy estacionario $(X, M)$ tales que $\lim _{n \rightarrow \infty} M\left(x_{n}, y_{n}\right)=0$. Esto no es posible cuando $*$ es íntegra como muestra el siguiente lema.

Lema 4.2.13. Sea $(X, M, *)$ un espacio métrico fuzzy y supongamos que * es integra. Sean $\left\{a_{n}\right\}_{n} y\left\{b_{n}\right\}_{n}$ dos sucesiones de Cauchy en $X$ y $t>0$. Si $\left\{M\left(a_{n}, b_{n}, t\right)\right\}_{n \in \mathbb{N}}$ converge (en la topología usual de $\mathbb{R}$ ) a c, entonces $c>0$.

\section{Demostración.}

Sean $\left\{a_{n}\right\}_{n}$ y $\left\{b_{n}\right\}_{n}$ sucesiones de Cauchy en $X$ y supongamos que

$$
\lim _{n \rightarrow \infty} M\left(a_{n}, b_{n}, t\right)=c .
$$

Dado $\varepsilon \in] 0,1\left[\right.$ podemos encontrar $p \in \mathbb{N}$ tal que $M\left(a_{r}, a_{s}, \frac{t}{3}\right)>1-\varepsilon$ y $M\left(b_{r}, b_{s}, \frac{t}{3}\right)>1-\varepsilon$ para todo $r, s \geq p$.

Pongamos $M\left(a_{p}, b_{p}, \frac{t}{3}\right)=a>0$ y $\delta=(1-\varepsilon) * a *(1-\varepsilon)>0$ ya que $*$ es íntegra. Entonces para $n \geq p$ tenemos

$$
\begin{gathered}
M\left(a_{n}, b_{n}, t\right) \geq M\left(a_{n}, a_{p}, \frac{t}{3}\right) * M\left(a_{p}, b_{p}, \frac{t}{3}\right) * M\left(b_{p}, b_{n}, \frac{t}{3}\right) \geq \\
\geq(1-\varepsilon) * a *(1-\varepsilon)=\delta
\end{gathered}
$$

y así

$$
\lim _{n \rightarrow \infty} M\left(a_{n}, b_{n}, t\right)=c \geq \delta>0 .
$$

Teorema 4.2.14. Sea $(X, M, *)$ un espacio métrico fuzzy fuerte y supongamos que * es integra. Si $\left\{x_{n}\right\}_{n \in \mathbb{N}} e\left\{y_{n}\right\}_{n \in \mathbb{N}}$ son sucesiones de Cauchy en $X$ y $t>0$ entonces $\left\{M\left(x_{n}, y_{n}, t\right)\right\}_{n \in \mathbb{N}}$ converge en $\left.] 0,1\right]$.

\section{Demostración.}


Sean $\left\{x_{n}\right\}_{n \in \mathbb{N}}$ e $\left\{y_{n}\right\}_{n \in \mathbb{N}}$ sucesiones de Cauchy en $X$ y sea $t>0$. Como $M\left(x_{n}, y_{n}, t\right) \in[0,1]$ para todo $n \in \mathbb{N}$, podemos encontrar una subsucesión $\left\{M\left(x_{n_{i}}, y_{n_{i}}, t\right)\right\}_{i}$ de $\left\{M\left(x_{n}, y_{n}, t\right)\right\}_{n}$ que converge a $c \in[0,1]$. Como $\left\{x_{n_{i}}\right\}_{i}$ e $\left\{y_{n_{i}}\right\}_{i}$ son subsucesiones de $\left\{x_{n}\right\}_{n \in \mathbb{N}}$ e $\left\{y_{n}\right\}_{n \in \mathbb{N}}$, respectivamente, también son sucesiones de Cauchy, y por el lema anterior, tenemos que $c>0$.

Sea $\varepsilon \in] 0,1[$ tal que $c-\varepsilon>0$ y $t>0$. Afirmamos que existe $p \in \mathbb{N}$ tal que $M\left(x_{m}, y_{m}, t\right)>c-\varepsilon$ para todo $m \geq p$. Para mostrar esto, elegimos $\left.\delta \in\right] 0,1[$ tal que $\delta * \delta *\left(c-\frac{\varepsilon}{2}\right)>c-\varepsilon$. Como $\left\{x_{n}\right\}_{n \in \mathbb{N}}$ e $\left\{y_{n}\right\}_{n \in \mathbb{N}}$ son sucesiones de Cauchy, existe $l \in \mathbb{N}$ tal que $M\left(x_{m}, x_{n_{i}}, t\right)>\delta$ y $M\left(y_{n_{i}}, y_{m}, t\right)>\delta$ para todo $m, i \geq l$ y también existe $q \in \mathbb{N}$ tal que $M\left(x_{n_{i}}, y_{n_{i}}, t\right)>c-\frac{\varepsilon}{2}$ para todo $i \geq q$ ya que $\left\{M\left(x_{n_{i}}, y_{n_{i}}, t\right)\right\}_{i}$, converge a $c$.

Tomemos $p=\max \{l, q\}$. Como $M$ es fuerte, tenemos que

$$
\begin{gathered}
M\left(x_{m}, y_{m}, t\right) \geq M\left(x_{m}, x_{n_{p}}, t\right) * M\left(x_{n_{p}}, y_{n_{p}}, t\right) * M\left(y_{n_{p}}, y_{m}, t\right) \geq \\
\geq \delta *\left(c-\frac{\varepsilon}{2}\right) * \delta>c-\varepsilon
\end{gathered}
$$

para todo $m \geq p$, y así nuestra afirmación ha sido probada.

Ahora veamos, por reducción al absurdo, que $\lim _{n \rightarrow \infty} M\left(x_{n}, y_{n}, t\right)=c$.

Si $c=1$, es obvio.

Supongamos que $c<1$.

Si $\left\{M\left(x_{n}, y_{n}, t\right)\right\}_{n}$ no converge a $c$, existirá $\delta>0$ con $] c-\delta, c+\delta[\subset] 0,1[$ de manera que una cantidad infinita de términos de la sucesión $\left\{M\left(x_{n}, y_{n}, t\right)\right\}_{n}$ estarán en el compacto $[0, c-\delta] \cup[c+\delta, 1]$, y por lo tanto existirá una subsucesión $\left\{M\left(x_{n_{j}^{\prime}}, y_{n_{j}^{\prime}}, t\right)\right\}_{j}$ de $\left\{M\left(x_{n}, y_{n}, t\right)\right\}_{n}$ tal que $\lim _{j \rightarrow \infty} M\left(x_{n_{j}^{\prime}}, y_{n_{j}^{\prime}}, t\right)=e$, 
con $e \neq c$ y $e>0$ por el Lema 4.2.13, ya que $\left\{x_{n_{j}^{\prime}}\right\}$ e $\left\{y_{n_{j}^{\prime}}\right\}$ son sucesiones de Cauchy en $X$. Supongamos que $c<e$.

Sea $\varepsilon^{\prime}<\frac{e-c}{3}$ de manera que $0<c-\varepsilon^{\prime}<c+\varepsilon^{\prime}<e-\varepsilon^{\prime}$. Por la afirmación anterior, aplicada a $e, \varepsilon^{\prime}>0$ y $t>0$, existe $p_{1} \in \mathbb{N}$ tal que

$$
M\left(x_{m}, y_{m}, t\right)>e-\varepsilon^{\prime}, \text { para todo } m \geq p_{1}
$$

Por otra parte, como $\left\{M\left(x_{n_{i}}, y_{n_{i}}, t\right)\right\}_{i}$ converge a $c$, existe $p_{2} \in \mathbb{N}$ tal que $\left.M\left(x_{n_{i}}, y_{n_{i}}, t\right) \in\right] c-\varepsilon^{\prime}, c+\varepsilon^{\prime}\left[\right.$, para todo $i \geq p_{2}$, lo que contradice a (4.1).

Si suponemos que $e<c$, podemos considerar $\left.\varepsilon^{\prime} \in\right] 0,1\left[\right.$ con $0<\varepsilon^{\prime}<\frac{c-\varepsilon}{3}$ y tal que $0<e-\varepsilon^{\prime}<e+\varepsilon^{\prime}<c-\varepsilon$.

Ahora, como $\left\{M\left(x_{n_{j}^{\prime}}, y_{n_{j}^{\prime}}, t\right)\right\}_{j}$ converge a $e$, existe $p_{3} \in \mathbb{N}$ tal que $\left.M\left(x_{n_{j}^{\prime}}, y_{n_{j}^{\prime}}, t\right) \in\right] e-\varepsilon^{\prime}, e+\varepsilon^{\prime}\left[\right.$ para todo $n_{j}^{\prime} \geq p_{3}$, lo cual es una contradicción con que $M\left(x_{m}, y_{m}, t\right)>c-\varepsilon$ para todo $m \geq p$.

Por consiguiente, $\left\{M\left(x_{n}, y_{n}, t\right)\right\}_{n}$ converge a $c$.

Recordar que (Proposición 3 de [23]) un espacio métrico fuzzy estacionario $(X, M, *)$ es completable si y sólo si $\lim _{n \rightarrow \infty} M\left(a_{n}, b_{n}\right)>0$ para cada par de sucesiones de Cauchy $\left\{a_{n}\right\}_{n},\left\{b_{n}\right\}_{n}$, en $X$. Así, por el Teorema 4.2.14, tenemos los siguientes corolarios.

Corolario 4.2.15. Si $(M, *)$ es una métrica fuzzy estacionaria sobre $X y *$ es integra entonces $(X, M, *)$ es completable.

Recordemos que la métrica fuzzy $M$ se dice que es una ultramétrica si $M(x, z, t) \geq \min \{M(x, y, t), M(y, z, t)\}$, para cualesquiera $x, y, z \in X$, 
$t>0$. Por tanto, si $(M, *)$ es una ultramétrica sobre $X$, entonces $*$ es necesariamente la t-norma mínimo, que es íntegra, con lo que como caso particular del Corolario 4.2.15, tenemos el siguiente corolario.

Corolario 4.2.16. Las ultramétricas fuzzy estacionarias son completables.

El Ejemplo 2 de [22] es un ejemplo de un espacio métrico fuzzy estacionario que no admite completación fuzzy.

Lema 4.2.17. Supongamos que $(X, M, *)$ es completable. Sea $\left(X^{*}, M^{*}, *\right)$ la completación fuzzy de $(X, M, *)$. Si $(M, *)$ es fuerte entonces $\left(M^{*}, *\right)$ es fuerte.

\section{Demostración.}

Sean $x, y, z, \in X^{*}$. Sean $\left\{x_{n}\right\}_{n \in \mathbb{N}},\left\{y_{n}\right\}_{n \in \mathbb{N}}$ y $\left\{z_{n}\right\}_{n \in \mathbb{N}}$ sucesiones en $X$ que convergen a $x, y, z$, respectivamente. Entonces, por construcción de $M^{*}$ [23] tenemos que

$$
\begin{gathered}
M^{*}(x, z, t)=\lim _{n \rightarrow \infty} M\left(x_{n}, z_{n}, t\right) \geq \lim _{n \rightarrow \infty}\left\{M\left(x_{n}, y_{n}, t\right) * M\left(y_{n}, z_{n}, t\right)\right\}= \\
=M^{*}(x, y, t) * M^{*}(y, z, t)
\end{gathered}
$$

ya que $M$ es continua sobre $X^{2} \times \mathbb{R}^{+}[48]$ y $*$ es continua sobre $[0,1]^{2}$, y así $M^{*}$ es fuerte.

Teorema 4.2.18. Sea $(X, M, *)$ un espacio métrico fuzzy fuerte. Si $X$ es completable entonces $\left(X, M_{t}, *\right)$ es completable para todo $t>0$.

\section{Demostración.}

Sea $\left(X^{*}, M^{*}, *\right)$ la completación métrica fuzzy de $(X, M, *)$. Por el lema anterior, $M^{*}$ es fuerte sobre $X^{*}$ y así por el Corolario 4.2.10 $M_{t}^{*}$ es una métrica fuzzy estacionaria completa sobre $X^{*}$ para cada $t>0$. 
Veamos que $\left(X^{*}, M_{t}^{*}, *\right)$ es la completación métrica fuzzy de $\left(X, M_{t}, *\right)$ para todo $t>0$.

Primero veamos que $X$ es denso en $X^{*}$ dotado con la topología $\tau_{M_{t}^{*}}$, para todo $t>0$.

Sea $x \in X^{*}$ y consideremos una sucesión $\left\{x_{n}\right\}_{n \in \mathbb{N}}$ en $X$ tal que $\left\{x_{n}\right\}_{n \in \mathbb{N}}$ converge a $x$ en $\tau_{M^{*}}$. Entonces, por (ii) de la Proposición 4.2.5 $\left\{x_{n}\right\}_{n \in \mathbb{N}}$ converge a $x$ en $\tau_{M_{t}^{*}}$ para todo $t>0$ y así $X$ es denso en $\left(X^{*}, \tau_{M_{t}^{*}}\right)$.

Claramente, la función identidad de $\left(X, M_{t}\right)$ en $\left(X, M_{t}^{*}\right)$ es una isometría, ya que, para cada $t>0$,

$$
M_{t}(x, y)=M(x, y, t)=M^{*}(x, y, t)=M_{t}^{*}(x, y) \text { para todo } x, y \in X .
$$

Así $\left(X^{*}, M_{t}^{*}, *\right)$ es la completación métrica fuzzy de $\left(X, M_{t}, *\right)$.

El siguiente ejemplo nos muestra que no podemos extender el Corolario 4.2.16 a ultramétricas fuzzy y también que el recíproco del teorema anterior es falso.

Ejemplo 4.2.19. Sean $\left\{x_{n}\right\}_{n \in \mathbb{N}} e\left\{y_{n}\right\}_{n \in \mathbb{N}}$ dos sucesiones estrictamente crecientes de números reales positivos, que convergen a 1 con respecto a la métrica euclídea, con $A \cap B=\phi$, donde $A=\left\{x_{n}: n \in \mathbb{N}\right\}$ y $B=\left\{y_{n}: n \in \mathbb{N}\right\}$. Sea $X=A \cup B$ y definimos sobre $X \times X \times] 0,+\infty[$ un conjunto fuzzy $M$ definido por

$$
\begin{aligned}
& M\left(x_{n}, x_{n}, t\right)=M\left(y_{n}, y_{n}, t\right)=1 \text { para todo } n \in \mathbb{N}, t>0, \\
& M\left(x_{n}, x_{m}, t\right)=x_{n} \wedge x_{m} \text { para todo } n, m \in \mathbb{N} \text { con } n \neq m, t>0, \\
& M\left(y_{n}, y_{m}, t\right)=y_{n} \wedge y_{m} \text { para todo } n, m \in \mathbb{N} \text { con } n \neq m, t>0,
\end{aligned}
$$


$M\left(x_{n}, y_{m}, t\right)=M\left(y_{m}, x_{n}, t\right)=x_{n} \wedge y_{m}$ para todo $n, m \in \mathbb{N}, t \geq 1$,

$M\left(x_{n}, y_{m}, t\right)=M\left(y_{m}, x_{n}, t\right)=x_{n} \wedge y_{m} \wedge t$ para todo $n, m \in \mathbb{N}, 0<t<1$.

$(X, M, \wedge)$ es un espacio métrico fuzzy que no es completable (Ejemplo 2 de [23]).

Veamos que $M$ es fuerte, y como conclusión se tendrá que $(X, M, \wedge)$ es un espacio ultramétrico fuzzy.

Abreviaremos la demostración obviando los casos triviales.

Supongamos que $0<t<1$. Tenemos

$$
M\left(x_{n}, x_{m}, t\right)=x_{n} \wedge x_{m} \geq x_{n} \wedge x_{r} \wedge x_{m}=M\left(x_{n}, x_{r}, t\right) * M\left(x_{r}, x_{m}, t\right)
$$

y de la misma forma

$$
\begin{gathered}
M\left(y_{n}, y_{m}, t\right) \geq M\left(y_{n}, y_{r}, t\right) * M\left(y_{r}, y_{m}, t\right), \text { para } n \neq m . \\
M\left(x_{n}, x_{m}, t\right)=x_{n} \wedge x_{m} \geq x_{n} \wedge y_{r} \wedge x_{m} \wedge t=M\left(x_{n}, y_{r}, t\right) * M\left(y_{r}, x_{m}, t\right)
\end{gathered}
$$

y de la misma forma

$$
\begin{gathered}
M\left(y_{n}, y_{m}, t\right) \geq M\left(y_{n}, x_{r}, t\right) * M\left(x_{r}, y_{m}, t\right), \text { para } n \neq m . \\
M\left(x_{n}, y_{m}, t\right)=x_{n} \wedge y_{m} \wedge t \geq x_{n} \wedge x_{r} \wedge y_{m} \wedge t= \\
=M\left(x_{n}, x_{r}, t\right) * M\left(x_{r}, y_{m}, t\right), \text { para } n \neq m .
\end{gathered}
$$

Si $r=n$ la desigualdad es obvia. De la misma forma

$$
M\left(x_{n}, y_{m}, t\right) \geq M\left(x_{n}, y_{r}, t\right) * M\left(y_{r}, y_{m}, t\right), \text { para } n \neq m \text {. }
$$

Si $r=m$ la desigualdad es obvia. 
El caso $t \geq 1$ es trivial.

Como $(X, M, \wedge)$ es fuerte entonces $\left(X, M_{t}, \wedge\right)$ son métricas fuzzy estacionarias y así completables para cada $t>0$ ya que $*$ es una t-norma continua integra.

Teorema 4.2.20. Sea $(X, M, *)$ un espacio métrico fuzzy fuerte y supongamos que * es integra. Entonces $\tau_{M}=\bigvee\left\{\tau_{M_{t}}: t>0\right\}$ donde cada uno de los espacios $\left(X, M_{t}, *\right)$ es completable.

\section{Demostración.}

Consideremos para todo $t>0$ la métrica fuzzy estacionaria $M_{t}$ sobre $X$ asociada a $M$.

Denotemos por $B_{M_{t}}(x, r)$ a la bola centrada en $x \in X$ y de radio $\left.r \in\right] 0,1[$ en $\left(X, M_{t}, *\right)$. Es fácil probar que para $t<s, B_{M_{t}}(x, r) \subset B_{M_{s}}(x, r)$, y por lo tanto $\tau_{M_{t}} \succ \tau_{M_{s}}$.

Por el Corolario 4.2.15 $\left(M_{t}, *\right)$ es completable para cada $t>0$, y finalmente, como $B_{M}(x, r, t)=B_{M_{t}}(x, r)$, se deduce inmediatamente que $\tau_{M}=\bigvee\left\{\tau_{M_{t}}: t>0\right\}$. 


\section{Capítulo 5}

\section{Aplicación de métricas fuzzy}

\subsection{Introducción}

En este capítulo, para ilustrar los beneficios prácticos que podemos obtener de las métricas fuzzy, damos una aplicación para filtrado de imagen en color. Veremos que los métodos de filtrado de imagen construidos usando métricas fuzzy funcionan mejor que otros métodos clásicos.

En la Sección 5.2 vemos una introducción a las imágenes digitales con el fin de dar a conocer los elementos que las forman, que nos serán de utilidad para los métodos de filtrado propuestos. Estos elementos que forman la imagen reciben el nombre de píxeles y cada uno de ellos está asociado con unas cantidades de colores primarios rojo, verde y azul, llamadas componentes de color RGB (Red, Green, Blue) de un píxel. Estas componentes indican la mezcla de colores primarios que se debe realizar para obtener el color de ese píxel. Cuando se forma una imagen digital, puede verse afectada por diversos 
factores que pueden empeorar la calidad de ésta, ya que usualmente pueden aparecer irregularidades, también llamadas ruido.

En la Sección 5.3 vemos cómo realizamos el filtrado utilizando métricas fuzzy. Se trata de sustituir un píxel con ruido, por otro libre de ruido que denotaremos por $\mathbf{F}_{i}$ y $\hat{\mathbf{F}}_{i}$, respectivamente. Para determinar $\hat{\mathbf{F}}_{i}$ utilizamos el principio de orden reducido, que determina, en un entorno cuadrado de tamaño $n$, el vector de color que es más similar en global a todos los demás vectores del entorno. Es decir, $\hat{\mathbf{F}}_{i}$ es el vector de color del entorno que maximiza la distancia fuzzy acumulada a todos los vectores de color del entorno.

Como primera aplicación a este método de filtrado, usamos las métricas fuzzy

- $G\left(x, y, k_{G}\right)=\frac{k_{G}}{k_{G}+|x-y|}$

- $E\left(x, y, k_{E}\right)=e^{-\frac{|x-y|}{k_{E}}}$

- $M\left(x, y, k_{M}\right)=\frac{\operatorname{mín}\{x, y\}+k_{M}}{\operatorname{máx}\{x, y\}+k_{M}}$

- $T\left(x, y, k_{T}\right)=1-\frac{|x-y|}{k_{T}}$

que son casos particulares de las métricas fuzzy (2.4), (2.6), (2.1) y (2.7) estudiadas en el capítulo 2, y comparamos el resultado del filtrado con el obtenido al utilizar las métricas clásicas $L_{2}$ y $L_{\infty}$.

En la segunda aplicación desarrollamos un método de filtrado utilizando una combinación de dos métricas fuzzy donde medimos simultáneamente la cercanía entre dos píxeles de la imagen y la similitud entre sus componentes de color RGB. Utilizando esta combinación, obtenemos mejores resultados 
ya que la inclusión de un criterio de medida espacial contribuye a mejorar la preservación de los detalles y bordes de la imagen, ya que, como explicaremos, el vector de salida es un vector espacialmente cercano al píxel original.

\subsection{Imágenes digitales}

Como podemos ver más detalladamente en [50], una imagen es una representación $2 D$ de los objetos que están en una escena $3 D$ y se obtiene mediante una proyección de los objetos $3 D$ sobre una imagen plana. En cámaras digitales, esta proyección se obtiene siguiendo el modelo de cámara de "ojo de la aguja" [19] y [46] (ver Figura 5.1(a)). De acuerdo con este modelo, los objetos representados en un espacio $3 D$, se proyectan a través de un centro de proyección sobre la imagen plana $2 D$. El centro de proyección corresponde a la óptica de la cámara. La imagen plana se representa físicamente por un sensor Charge Couple Device (CCD) que capta la radiación luminosa. Este sensor (CCD) (ver Figura 5.1(b)) comprende un array de $M \times N$ sensores de luz simples. Así, cuando se toma una fotografía con una cámara digital, se captura una representación del mundo $3 D$ en un array de $M \times N$ elementos simples que reciben el nombre de píxeles.

En imágenes digitales en color, cada píxel representa un único color de la imagen. Los colores se representan comunmente en los ordenadores usando el espacio de color Rojo-Verde-Azul (Red-Green-Blue (RGB)). Este espacio de color sigue un modelo aditivo de modo que cualquier color se obtiene mediante la mezcla adecuada de los tres primarios: rojo, verde y azul. Así, cada píxel de la imagen en color se asocia con una terna de valores (RGB) que representan la cantidad apropiada de cada color primario que se debe mezclar para obtener el color almacenado en ese píxel. Desde un punto de vista 


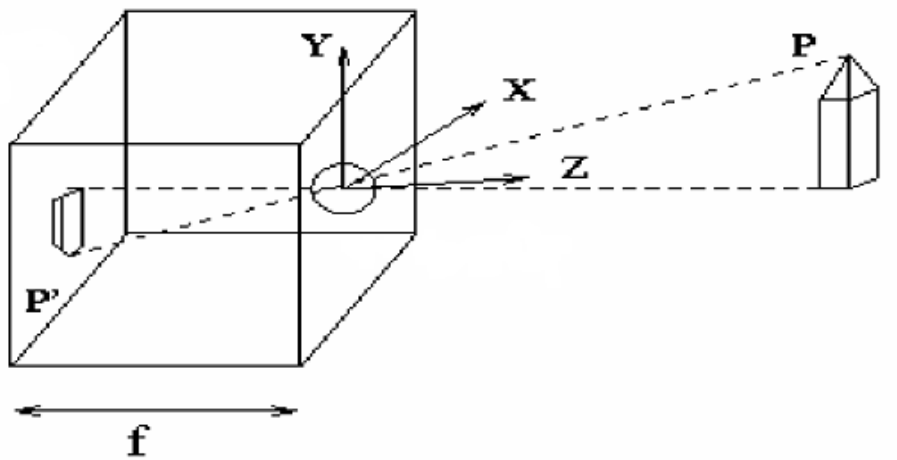

(a)

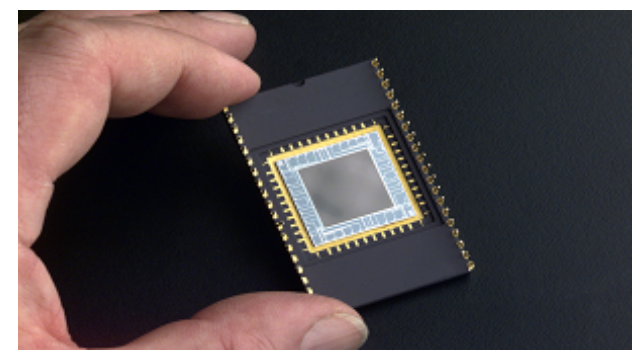

(b)

Figura 5.1: (a) Esquema del modelo de cámara Ojo Aguja: El objeto $P$, representado en el espacio $(X, Y, Z)$, se proyecta a través del centro de proyección (óptica de la cámara) sobre la imagen plana (CCD) como el objeto $P^{\prime}$. (b) Un sensor CCD.

geométrico, cada color también se puede interpretar como un punto de un espacio tridimensional cuyas coordenadas son las cantidades correspondientes a cada uno de los colores primarios.

El proceso de la adquisición de la imagen digital puede verse afectado por diferentes factores que pueden degradar la calidad de la imagen. Por ejemplo, condiciones adversas de iluminación o mal funcionamiento del sensor CCD pueden introducir irregularidades en la imagen conocidas como ruido. Otros 
factores capaces de afectar a la calidad de imagen son, entre otros, errores de transmisión o defectos de almacenamiento. La Figura 5.2 muestra una imagen ideal libre de ruido (a) y la misma imagen contaminada con ruido (b). La presencia de irregularidades, o ruido, en una imagen son, en general, indeseables, tanto el punto de vista de la calidad visual de la imagen como para el procesamiento automático de ésta, ya que la presencia de ruido puede ser un grave impedimento para el correcto funcionamiento de las tareas de procesamiento automático de la imagen.

\subsection{Filtrado de imagen utilizando métricas fuzzy}

Denotemos por $\mathbf{F}$ a la imagen digital que vamos a tratar. Procesamos la imagen empleando un enfoque de ventana deslizante: para procesar cada píxel de la imagen, tomamos los píxeles dentro de un entorno cuadrado $W$ de tamaño $n \times n, n=3,5,7, \ldots$, centrado en él.

Denotamos por $\mathbf{F}_{i}, i=0, \ldots, n^{2}-1$, los $n^{2}$ vectores de color asociados a los píxeles en $W$. Nótese que un píxel de una imagen en color se caracteriza, como hemos visto en la sección anterior, por una terna que define sus componentes de color RGB, que es, $\mathbf{F}_{i}=\left\{F_{i}^{R}, F_{i}^{G}, F_{i}^{B}\right\}$.

En este contexto, podemos medir la distancia fuzzy entre dos vectores de color mediante la conjunción con una adecuada t-norma de las distancias fuzzy entre sus componentes de color. A continuación detallamos el proceso de filtrado y mostramos cómo las métricas fuzzy pueden sustituir a las métricas clásicas en este proceso, y cuáles son los beneficios resultantes de esta sustitución. 
El problema de filtrado de una imagen digital implica eliminar los píxeles ruidosos y reemplazarlos por otros libres de ruido. Dentro de un enfoque de ventana deslizante, esto se puede hacer mediante la identificación en $W$ de un píxel libre de ruido $\hat{\mathbf{F}}_{i}$ para sustituir el original $\mathbf{F}_{i}$. En un contexto vectorial, esta identificación puede hacerse mediante el empleo del principio de orden reducido, que determina cuál de los vectores de color en la población es el más similar, en general, a todos los demás. En particular, $\hat{\mathbf{F}}_{i}$ se puede determinar como el vector de color en la población que maximiza la distancia fuzzy acumulada a todos los otros vectores de color en $W$, de la siguiente manera.

Tomando $C$ como la métrica fuzzy para maximizar, para cada vector $\mathbf{F}_{k}$ en la ventana $W$, calculamos una medida acumulada

$$
A_{\mathbf{k}}=\sum_{j \in W, j \neq k} C\left(\mathbf{F}_{k}, \mathbf{F}_{j}, t\right)
$$

a todos los otros vectores de la ventana. Los valores $A_{\mathbf{k}}$ se ordenan en una sucesión decreciente (en la que el valor situado en la posición r-ésima se escribe como $\left.A_{(r)}\right)$ de la siguiente forma:

$$
A_{(0)} \geq A_{(1)} \geq \cdots \geq A_{\left(n^{2}-1\right)} .
$$

Este orden implica el mismo orden de los vectores $\mathbf{F}_{\mathbf{k}}$ 's:

$$
\mathbf{F}_{(0)} \geq \mathbf{F}_{(1)} \geq \cdots \geq \mathbf{F}_{\left(n^{2}-1\right)} .
$$

Entonces la salida del filtro $\hat{\mathbf{F}}_{i}$ será el vector $\mathbf{F}_{(0)}$ que ocupa la posición menor en la sucesión ordenada.

En un contexto análogo, el conocido Vector Median Filter (VMF) emplea la métrica euclídea $\left(L_{2}\right)$ como el criterio de distancia entre los vectores. Además, otra métrica popular es métrica $L_{\infty}$ donde la distancia entre dos vectores es el máximo del valor absoluto de las diferencias entre sus componentes. 


\subsection{Primera aplicación}

Como una primera aplicación de métricas fuzzy para filtrado de imagen en color, usamos las siguientes métricas fuzzy, que son casos particulares de las métricas fuzzy (2.4), (2.6), (2.1) y (2.7) estudiadas en el capítulo 2, con el anterior método de filtrado explicado.

- $G\left(x, y, k_{G}\right)=\frac{k_{G}}{k_{G}+|x-y|}$

- $E\left(x, y, k_{E}\right)=e^{-\frac{|x-y|}{k_{E}}}$

- $M\left(x, y, k_{M}\right)=\frac{\operatorname{mín}\{x, y\}+k_{M}}{\operatorname{máx}\{x, y\}+k_{M}}$

- $T\left(x, y, k_{T}\right)=1-\frac{|x-y|}{k_{T}}$

Para $G, E$ y $M$, tomamos el producto usual como t-norma de la operación binaria, pero para $T$ tomamos la t-norma $\mathfrak{L}$ de Lukasiewicz porque $T$ no es una métrica fuzzy con el producto usual. Posteriormente, comparamos el rendimiento de estas métricas fuzzy con la distancia euclidea clásica $\left(L_{2}\right)$ y con la métrica $L_{\infty}$.

El proceso experimental común para evaluar diferentes métodos de filtrado es tomar una imagen original libre de ruido (Figura 5.2 (a)), simular su contaminación mediante un modelo de ruido impulsivo (Figura 5.2 (b)) y filtrar la imagen (Figura 5.2 (c)) para recuperar la original. Las medidas de calidad objetivas se utilizan para determinar el grado de calidad de la imagen obtenida. Nosotros usamos el Pico Relación Señal Ruido (PSNR) definido de la siguiente forma 


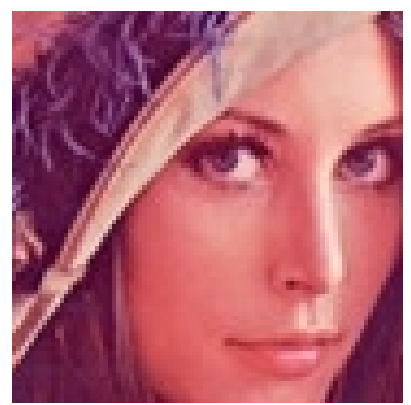

(a)

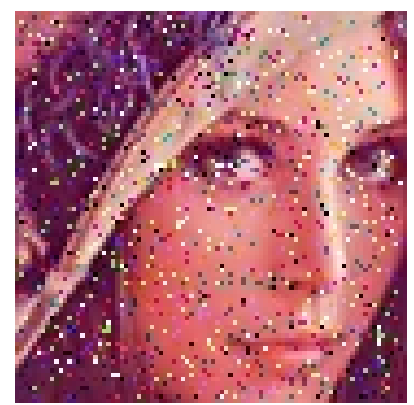

(b)

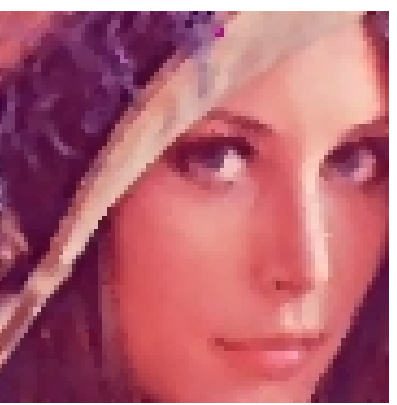

(c)

Figura 5.2: Proceso del experimento: (a) Imagen sin ruido, (b) Imagen con ruido, (c) Imagen filtrada.

$$
P S N R=20 \log \left(\frac{255}{\sqrt{\frac{1}{N M Q} \sum_{i \in \mathbf{F}} \sum_{q=1}^{Q}\left(\mathbf{F}_{i}^{q}-\hat{\mathbf{F}}_{i}^{q}\right)^{2}}}\right)
$$

donde $M$ y $N$ son las dimensiones de la imagen $\mathbf{F}, Q$ es el número de canales de la imagen $\left(Q=3\right.$ para imágenes en color), y $\mathbf{F}_{i}^{q}$ y $\hat{\mathbf{F}}_{i}^{q}$ denotan la componente q-ésima de los vectores de la imagen original y de la imagen filtrada, en la posición $i$, respectivamente.

Usamos cuatro imágenes de prueba diferentes, que contaminamos con porcentajes de ruido de $0 \%$ a $50 \%$, con un incremento del $5 \%$. Todas las imágenes ruidosas se filtran con el método descrito anteriormente utilizando las dos métricas clásicas y las cuatro métricas fuzzy consideradas. Luego, calculamos el valor PSNR medio para las cuatro imágenes en cada caso.

En los resultados experimentales (Cuadro 5.1 y Cuadro 5.2) podemos ver que todas las métricas fuzzy estudiadas superan a las dos métricas clásicas, 


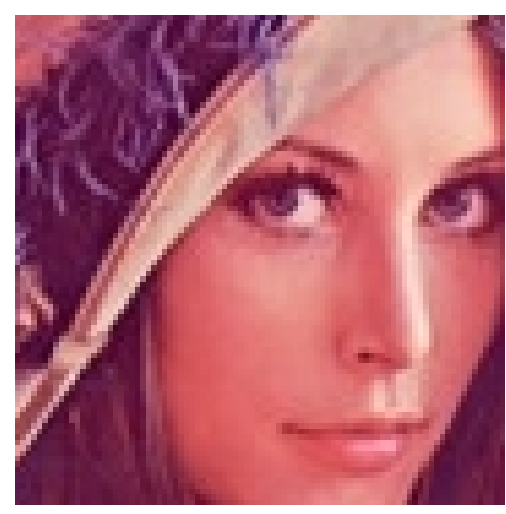

(a)

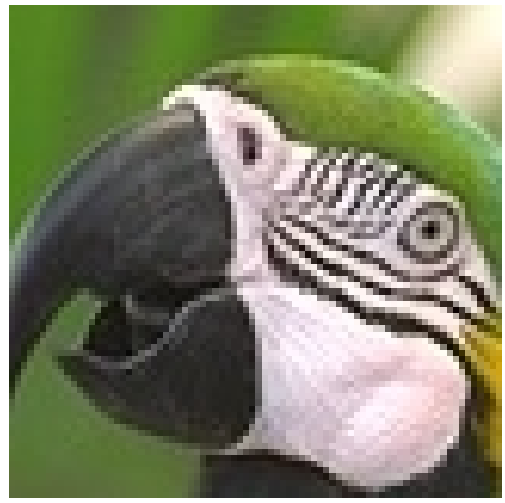

(c)

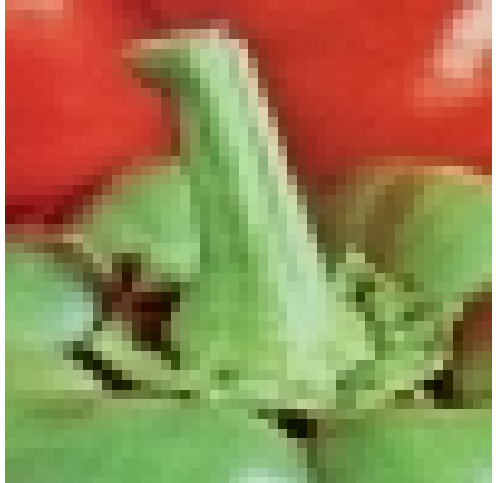

(b)

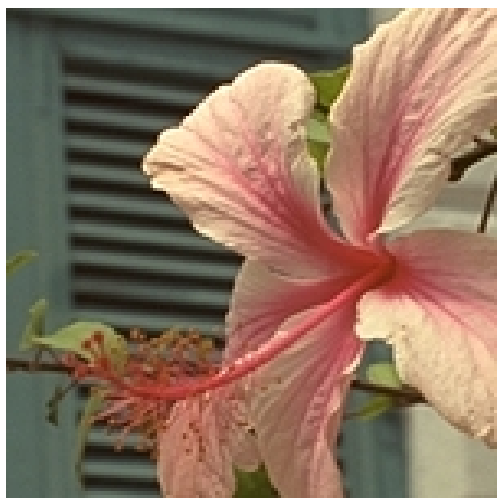

(d)

Figura 5.3: Imágenes de prueba

especialmente para alta densidad de ruido. También, se observa que todas las métricas fuzzy funcionan de manera similar. De todo esto, podemos concluir que la mejora obtenida no se debe a la forma de la función utilizada, sino a la conjunción hecha con t-normas para medir la distancia fuzzy entre los vectores de color. En la conjunción, el valor fuzzy más bajo es el que pesa más en el cálculo de la distancia fuzzy final dada por la métrica, pero sin despreciar el resto de los valores. Como el ruido está asociado, al menos, al valor fuzzy más bajo, se detecta mejor y el proceso de filtrado es más eficaz. 
Cuadro 5.1: Comparación de rendimiento en términos de PSNR variando el porcentaje de ruido (bajo)

\begin{tabular}{|c|cccccc|}
\hline Filtro & $0 \%$ & $5 \%$ & $10 \%$ & $15 \%$ & $20 \%$ & $25 \%$ \\
\hline$L_{2}$ & 27.86 & 27.40 & 27.12 & 26.78 & 26.34 & 26.09 \\
$L_{\infty}$ & 27.82 & 27.23 & 26.54 & 26.18 & 25.46 & 25.14 \\
$G$ & 27.80 & 27.64 & 27.45 & 27.32 & 26.83 & 26.75 \\
$E$ & 27.80 & 27.64 & 27.41 & 27.30 & 26.84 & 26.73 \\
$M$ & 27.81 & 27.64 & 27.45 & 27.32 & 26.83 & 26.73 \\
$T$ & 27.80 & 27.65 & 27.45 & 27.28 & 26.85 & 26.70 \\
\hline
\end{tabular}

Cuadro 5.2: Comparación de rendimiento en términos de PSNR variando el porcentaje de ruido (alto)

\begin{tabular}{|c|ccccc|}
\hline Filtro & $30 \%$ & $35 \%$ & $40 \%$ & $45 \%$ & $50 \%$ \\
\hline$L_{2}$ & 25.46 & 25.25 & 24.64 & 23.96 & 23.29 \\
$L_{\infty}$ & 24.27 & 23.96 & 23.22 & 22.29 & 21.57 \\
$G$ & 26.23 & 25.94 & 25.51 & 24.68 & 24.06 \\
$E$ & 26.20 & 25.92 & 25.55 & 24.72 & 24.02 \\
$M$ & 26.20 & 25.93 & 25.56 & 24.72 & 24.05 \\
$T$ & 26.18 & 25.87 & 25.55 & 24.68 & 24.07 \\
\hline
\end{tabular}

\subsection{Segunda aplicación}

Ahora, consideremos que un píxel de una imagen de color se caracteriza por su posición en la imagen $\mathbf{q}=\left\{q_{1}, q_{2}\right\}$, dada por dos coordenadas de posición, y por una terna que define su componente de color RGB, que es, $\mathbf{F}_{\mathbf{q}}=\left\{F_{\mathbf{q}}^{R}, F_{\mathbf{q}}^{G}, F_{\mathbf{q}}^{B}\right\}$. En este contexto, podemos medir la distancia entre dos vectores de color atendiendo a la similitud de sus componentes de color, y también atendiendo a la proximidad espacial entre los píxeles. Proponemos utilizar una métrica fuzzy que representa simultaneamente ambos criterios de distancia. Para ello, por un lado, usamos la métrica fuzzy $R$ dada por 


$$
R\left(\mathbf{F}_{\mathbf{i}}, \mathbf{F}_{\mathbf{j}}\right)=\prod_{l=1}^{3} \frac{\min \left\{F_{\mathbf{i}}^{l}, F_{\mathbf{j}}^{l}\right\}+K}{\max \left\{F_{\mathbf{i}}^{l}, F_{\mathbf{j}}^{l}\right\}+K},
$$

para medir la similitud entre los vectores de color $\mathbf{F}_{\mathbf{i}}$ y $\mathbf{F}_{\mathbf{j}}$. En esta expresión, establecemos $K=1024$ ya que en investigaciones anteriores se ha demostrado que es un valor adecuado para procesar imágenes de color RGB [39].

Por otro lado, la proximidad espacial entre dos píxeles de la imagen $\mathbf{F}_{\mathbf{i}}$ y $\mathbf{F}_{\mathbf{j}}$ está dada por la métrica fuzzy $S$ definida por

$$
S\left(\mathbf{F}_{\mathbf{i}}, \mathbf{F}_{\mathbf{j}}, t\right)=\frac{t}{t+\|\mathbf{i}-\mathbf{j}\|},
$$

donde $\|\cdot\|$ denota la norma Euclídea y $t$ es un parámetro capaz de ajustar la sensibilidad de la métrica fuzzy. Ahora, construimos el producto de las anteriores métricas fuzzy $R$ y $S$ en una única métrica fuzzy $C$ dada por $C\left(\mathbf{F}_{\mathbf{i}}, \mathbf{F}_{\mathbf{j}}, t\right)=R\left(\mathbf{F}_{\mathbf{i}}, \mathbf{F}_{\mathbf{j}}\right) \cdot S\left(\mathbf{F}_{\mathbf{i}}, \mathbf{F}_{\mathbf{j}}, t\right)$. Se puede probar que el producto de métricas fuzzy es también una métrica fuzzy [49]. De este modo, la métrica fuzzy $C$ mide el parecido entre dos píxeles de la imagen teniendo en cuenta simultáneamente la similitud entre los componentes de color y la cercanía espacial de los píxeles. Así, nos proponemos utilizar esta métrica fuzzy combinada, en el método de filtrado explicado en la sección anterior.

Como hemos mencionado anteriormente, el clásico Vector Median Filter (VMF) [2] emplea la métrica Euclídea como criterio de distancia entre vectores. Este filtro es conocido por su comportamiento robusto pero las imágenes resultantes son a menudo demasiado suavizadas, y por consiguiente, los bordes y los detalles pequeños no están bien conservados. Por otro lado, en el enfoque que proponemos, podemos ver que la inclusión del criterio espacial 
contribuye a mejorar la conservación de los detalles de la imagen mientras que el ruido también se reduce. Esto se logra porque como vector de salida en cada ventana de filtrado se determina un vector que es espacialmente cercano a todos los otros vectores de la ventana. Por lo tanto, evitamos la posibilidad de sustituir un píxel por otro situado lejos de él, lo cual no es apropiado para conservar los bordes y los detalles. Las Figuras 5.4 - 5.6 muestran tres imágenes ruidosas filtradas con el VMF y el método propuesto. Podemos ver que ambos métodos son capaces de suprimir el ruido, pero, mientras que el VMF genera imágenes de salida demasiado borrosas, el método propuesto es capaz de conservar mejor los bordes de la imagen y los detalles, y así, mejorar la calidad visual de las imágenes obtenidas.

Finalmente, podemos concluir que las métricas fuzzy son adecuadas para el filtrado de imágenes en color y merece la pena investigar su utilidad en otras tareas de procesamiento de imágenes o en problemas de ingeniería. 


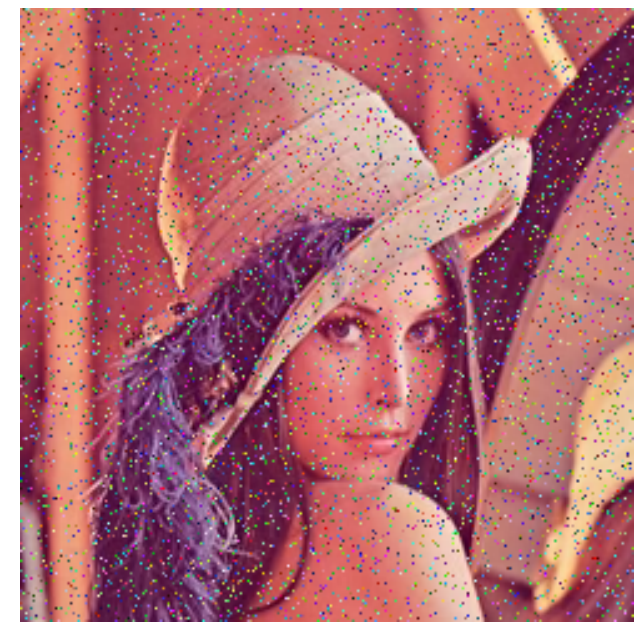

(a)

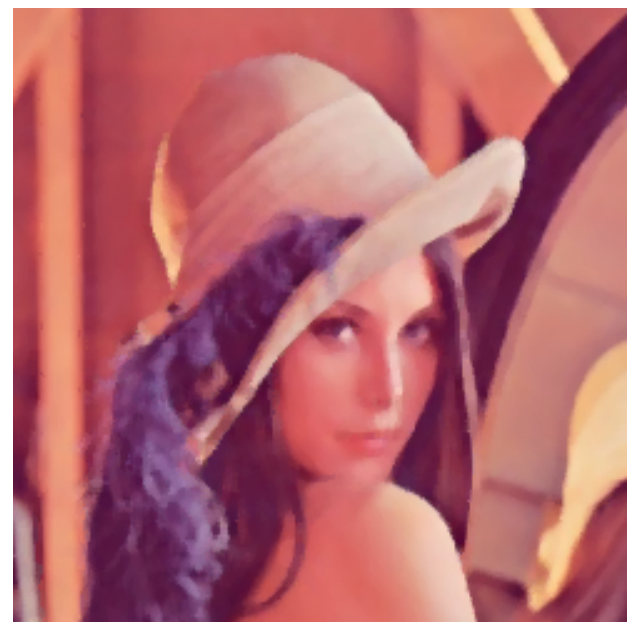

(b)

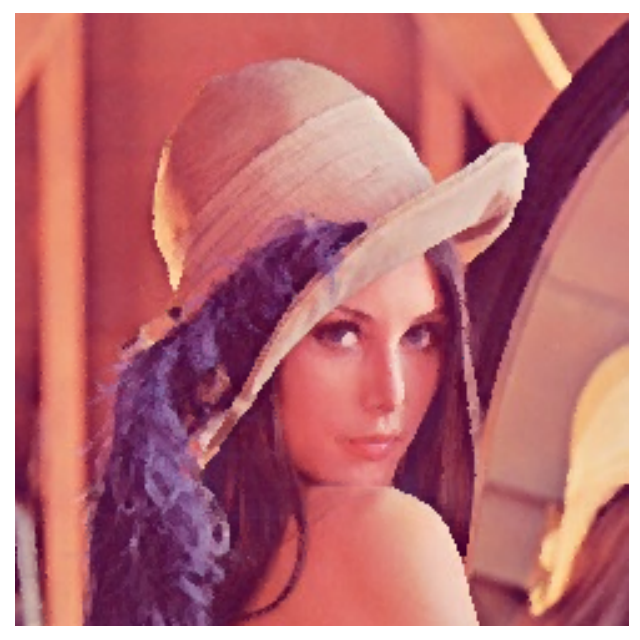

(c)

Figura 5.4:

Comparación de funcionamiento: (a) Detalle de la imagen Lenna donde el $10 \%$ de los píxeles son ruidosos, (b) Resultado obtenido usando el VMF [2] con un filtro ventana de tamaño $5 \times 5$, (c) Resultado obtenido con el método propuesto. 


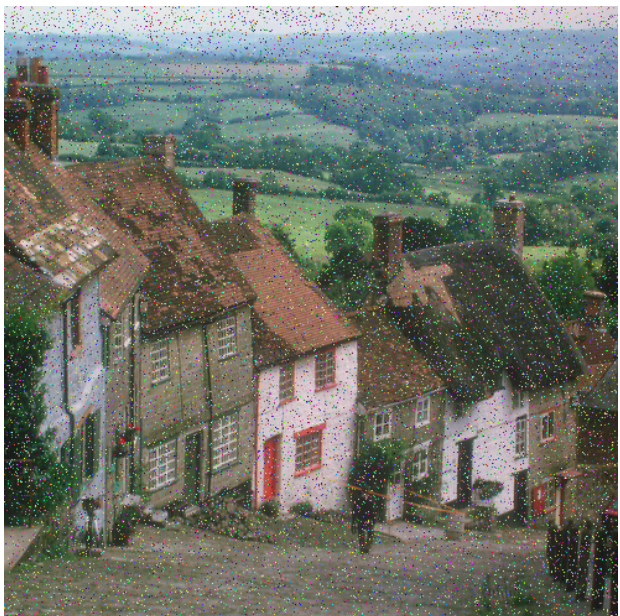

(a)

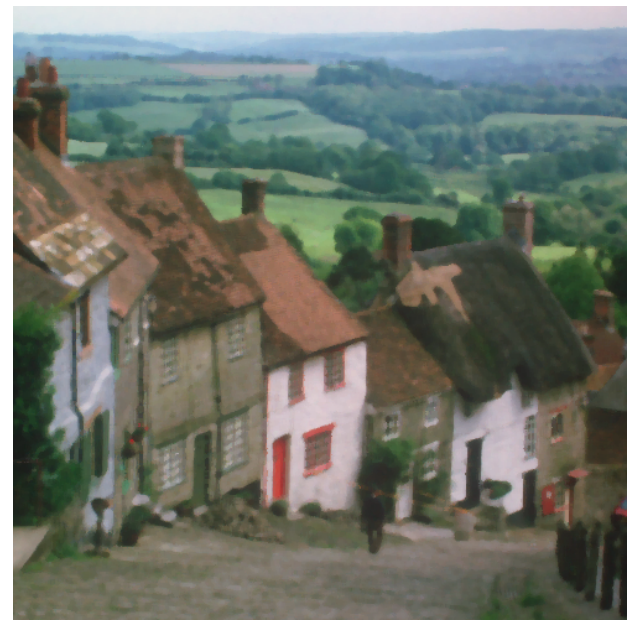

(b)

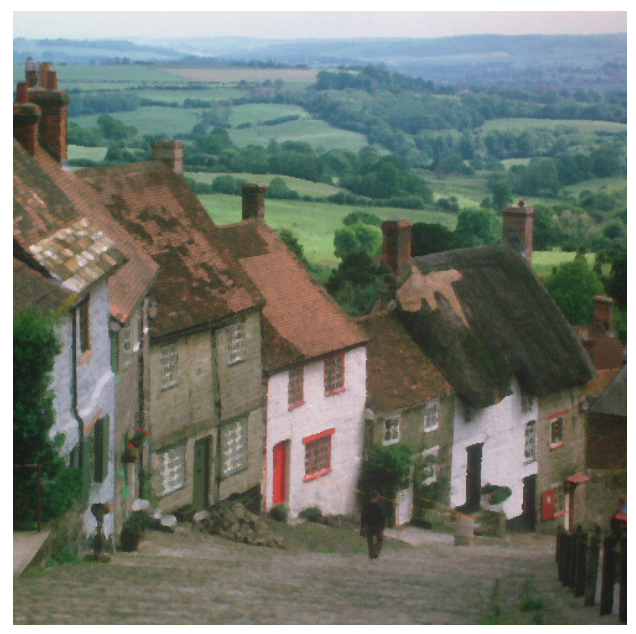

(c)

Figura 5.5:

Comparación de funcionamiento: (a) Detalle de la imagen Goldhill donde el $10 \%$ de los píxeles son ruidosos, (b) Resultado obtenido usando el VMF [2] con un filtro ventana de tamaño $5 \times 5$, (c) Resultado obtenido con el método propuesto. 


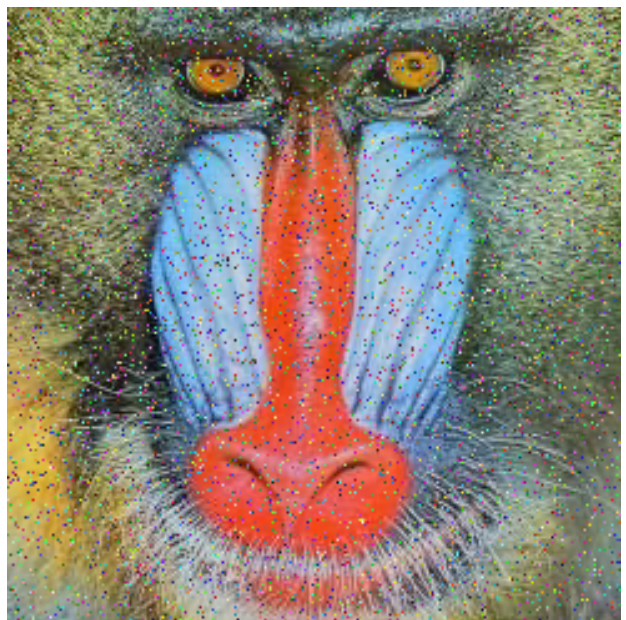

(a)

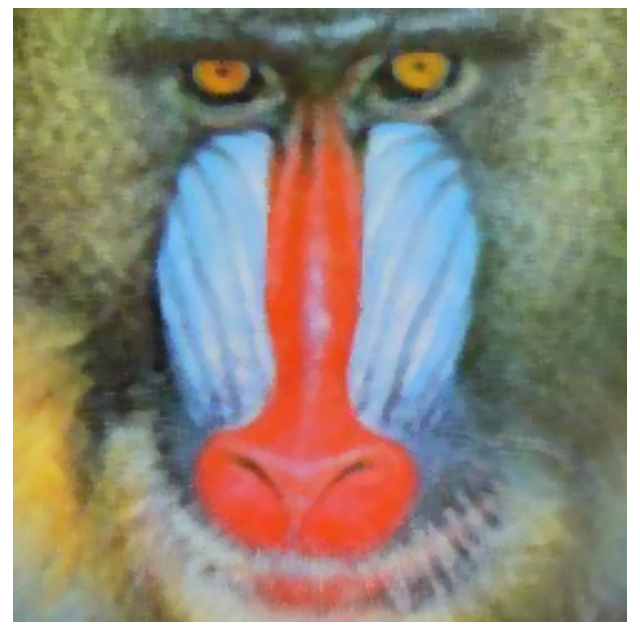

(b)

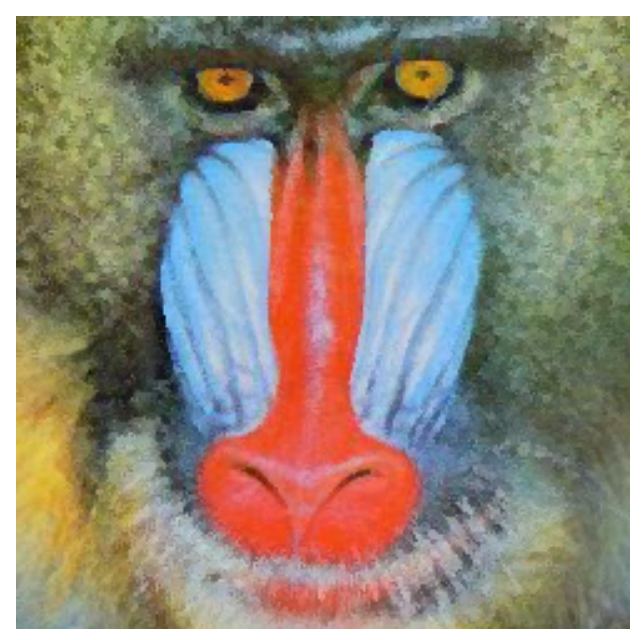

(c)

Figura 5.6:

Comparación de funcionamiento: (a) Detalle de la imagen Baboon donde el $10 \%$ de los píxeles son ruidosos, (b) Resultado obtenido usando el VMF [2] con un filtro ventana de tamaño $5 \times 5$, (c) Resultado obtenido con el método propuesto. 


\section{Bibliografía}

[1] G. Artico, R. Moresco, On fuzzy metrizability, J. Math. Anal. Appl. 107 (1985), 144-147.

[2] J. Astola, P. Hanvisto, Y. Neuvo, Vector median filters, Precedings of the IEEE 78 (April 1990), 678-689.

[3] K. Atanassov, Intuitionistic fuzzy sets, Fuzzy Sets and Systems 20 (1968), 87-96.

[4] K. Atanassov, Intuitionistic fuzzy sets, V.Sgurev, ed., VII ITKR's Session, Sofia (June 1983 Central Sci. and Techn. Library, Bulg. Academy of Sciences), 1984.

[5] K. Atanassov, Review and new results on intuitionistic fuzzy sets, Preprint IM-MFAIS-1-88, Sofia (1988).

[6] G. L. Cain JR., R. H. Karsiell, Fixed and periodic points of local contraction mappings on probabilistic metric spaces, Mathematical Systems Theory 9 (1976), 289-297.

[7] Joan-Gerard Camarena, V. Gregori, S. Morillas, A. Sapena, Fast detection and removal of impulsive noise using peer groups and fuzzy 
metrics, Journal of Visual Communication and Image Representation 19 (2008), 20-20.

[8] C. L. Chang, Fuzzy topological spaces, J. Math. Anal. Appl. 24 (1968), 182-190.

[9] K. C. Chattopadhyay, R. N. Hazra, S. K. Samanta, Gradation of openess: fuzzy topology, Fuzzy Sets and Systems 49 (1992), 237-242.

[10] D. ÇOKER, An introduction to intuitionistic fuzzy topological spaces, Fuzzy Sets and Systems 88 (1997), 81-89.

[11] P. Eklund, W. Gähler, Basic notions for fuzzy topology I, II, Fuzzy Sets and Systems 26 (1988), 333-356; 27 (1988) 171-195.

[12] R. Engelking, General Topology, PWN-Polish Sci. Publ., Warsawa, 1977.

[13] M. A. Erceg, Metric spaces in fuzzy set theory, J. Math. Anal. Appl. 69 (1979), 205-230.

[14] A. George, P. Veeramani, On some results in fuzzy metric spaces, Fuzzy Sets and Systems 64 (1994), 395-399.

[15] A. George, P. Veeramani, Some theorems in fuzzy metric spaces, J. Fuzzy Math. 3 (1995), 933-940.

[16] A. George, P. Veeramani, On some results of analysis for fuzzy metric spaces, Fuzzy Sets and Systems 90 (1997), 365-368.

[17] A. George, P. Veeramani, Compact and bounded sets in fuzzy metric spaces, J. Fuzzy Math. 8 (2000), 975-980.

[18] J. Goguen, L-fuzzy sets, J. Math. Anal. Appl. 18 (1967), 145-174. 
[19] R.C. González, R.E. Woods, Digital Image Processing, Prentice Hall, 2002.

[20] M. Grabiec, Fixed points in fuzzy metric spaces, Fuzzy Sets and Systems 27 (1988), 385-389.

[21] V. Gregori, S. Romaguera, Some properties of fuzzy metric spaces, Fuzzy Sets and Systems 115 (2000), 485-489.

[22] V. Gregori, S. Romaguera, On completion of fuzzy metric spaces, Fuzzy Sets and Systems 130 (2002), 399-404.

[23] V. Gregori, S. Romaguera, Caracterizing completable fuzzy metric spaces, Fuzzy Sets and Systems 144 (2004), 411-420.

[24] V. Gregori, S. Romaguera, A. Sapena, On t-uniformly continuous mappings in fuzzy metric spaces, The Journal of Fuzzy Mathematics. Los Angeles 12 (2004), 237-243.

[25] V. Gregori, S. Romaguera, P. Veeramani, A note on intuitionistic fuzzy metric spaces, Portugal. Math. 54 (1997), 431-440.

[26] V. Gregori, A. López-Crevillén, S. Morillas, A. Sapena, On convergence in fuzzy metric spaces, Topology and its applications 156 (2009), 3002-3006.

[27] V. Gregori, S. Morillas, A. López-Crevillén, On continuity and uniform continuity in fuzzy metric spaces, Proceedings of the Workshop in Applied Topology WiAT’09 (2009), 85-91.

[28] V. Gregori, A. Sapena, On fixed point theorems in fuzzy metric spaces, Fuzzy Sets and Systems 125 (2002), 245-252.

[29] R. N. Hazra, S. K. Samanta, K. C. Chattopadhyay, Fuzzy topology redefined, Fuzzy Sets and Systems 45 (1992), 79-82. 
[30] H. Hu, Fuzzy topological spaces, J. Math. Anal. Appl. 110 (1985), 141178.

[31] B. Hutton, Products of fuzzy topological spaces, Topology Appl. 11 (1980), 59-67.

[32] I. Jermolaeva, On a Hausdorffness function of a fuzzy topological space, Zbornik Radova Filozof. Fakulteta u Nižu. Ser. Mat. 2 (1988), 73.

[33] O. Kaleva, S. Seikkala, On fuzzy metric spaces, Fuzzy Sets and Systems 12 (1984), 215-229.

[34] J.C. KelLey, General Topology, Van Nostrand, 1955.

[35] I. Kramosil, J. Michalek, Fuzzy metric and statistical metric spaces, Kybernetika 11 (1975), 326-334.

[36] R. Lowen, Fuzzy topological spaces and fuzzy compactness, J. Math. Anal. Appl. 56 (1976), 621-633.

[37] R. Lowen, Initial and final fuzzy topologies and the fuzzy Tychonoff theorem, J. Math. Anal. Appl. 58 (1977), 11-21.

[38] D. Minet, On fuzzy contractive mappings in fuzzy metrics spaces, Fuzzy Sets and Systems 158 (2007), 915-921.

[39] S. Morillas, V. Gregori, G. Perís-Fajarnés, P. Latorre, A new vector median filter based on fuzzy metrics, Lecture Notes in Computer Science 3656 (2005), 81-90.

[40] S. Morillas, V. Gregori, G. Perís-Fajarnés, P. Latorre, A fast impulsive noise color image filter using fuzzy metrics, Real-Time Imaging 11 (2005), 417-428. 
[41] S. Morillas, V. Gregori, G. Perís-Fajarnés, A. Sapena, New adaptive vector filter using fuzzy metrics, Journal of Electronic Imaging 16 (2007), 033007:15pp.

[42] S. Morillas, V. Gregori, G. Perís-Fajarnés, A. Sapena, local self-adaptive fuzzy filter for impulsive noise removal in color images, Signal Precessing 88 (2008), 390-398.

[43] E. Pap, O. Hadzic, R. Mesiar, A fixed point theorem in probabilistic metric spaces and an application, J. Math. Anal. Appl. 202 (1996), 433449.

[44] E. PARAu, V. RADu, Some remarks on Tardiff's fixed point theorem on Menger spaces, Portugal. Math. 54 (1997), 431-440.

[45] J. H. PARK, Intuitionistic fuzzy metric spaces, Chaos, Solitons \& Fractals 22 (2004), 1039-1046.

[46] K.N. Plataniotis, A.N. Venetsanopoulos, Color Image processing and applications, Springer-Verlag, 2000.

[47] V. RAFu, Some fixed point theorems in probabilistic metric spaces, stability problems for stochastic models, Lecture Notes in Mathematics 1233, Springer, Berlin, (1987) 125-133.

[48] J. Rodríguez-López, S. Romaguera, The hausdorff fuzzy metric on compact sets, Fuzzy Sets and Systems 147 (2004), 273-283.

[49] A. Sapena, A contribution to the study of fuzzy metric spaces, Appl. Gen. Topology 2 (2001), 63-76.

[50] A. Sapena, S. Morillas, J. Camacho, Derivate-based optimization in colour image filtering: an application for derivative learning, Modelling in Science Education an Learning 2, (2009), 45-55. 
[51] B. Schweizer, A. Sklar, Statistical metric spaces, Pacific. J. Math. 10 (1960), 314-334.

[52] A. P. Šostak, On a fuzzy topological structure, Suppl. Rend. Circ. Mat. Palermo Ser II, 11 (1985) 89-103.

[53] A. P. Šostak, Two decades of fuzzy topology: basic ideas, notions and results, Uspekhi Mat. Nauk 44 (1989), 99-147.

[54] R. M. TARDIFF, Contraction maps on probabilistic metric spaces, J. Math. Anal. Appl. 165 (1992), 517-523.

[55] P. Veeramani, Best approximation in fuzzy metric spaces, J. Fuzzy Math. 9 (2001), 75-80.

[56] L.A. ZADEH, Fuzzy sets, Inform. Control 8 (1965), 338-353.

[57] Deng ZI-Ke, Fuzzy pseudo metric spaces, J. Math. Anal. Appl. 86 (1982), 74-95.

[58] Deng ZI-Ke, Separation axioms for completeness and total boundedness in fuzzy pseudometric spaces, J. Math. Anal. Appl. 112 (1985), 141-150. 